\title{
Total Synthesis and Determination of the Absolute Configuration of Parimycin
}

\author{
Day-Shin Hsu,* and Jiun-Yi Huang
}

Department of Chemistry and Biochemistry, National Chung Cheng University, Minhsiung 621, Taiwan chedsh@ccu.edu.tw

\section{Supporting Information}

1. General information $\quad$ S2

2. The comparison table for the ${ }^{1} \mathrm{H}$ NMR data of natural (+)-parimycin, synthetic ( \pm )-1, and (-)-1 S3

3. The comparison table for the ${ }^{13} \mathrm{C}$ NMR data of natural (+)-parimycin and synthetic ( \pm )-1 $\quad$ S4

4. Experimental section $\quad$ S5

$\begin{array}{ll}\text { 5. References } & \text { S21 }\end{array}$

6. ${ }^{1} \mathrm{H}$ and ${ }^{13} \mathrm{C}$ NMR spectra of compounds $\quad$ S22

$\begin{array}{lr}\text { 7. HPLC charts for }( \pm)-\mathbf{1 2} \text {, and }(R)-\mathbf{1 2} & \text { S59 }\end{array}$

8. HPLC charts for ( \pm -1, and (-)-1 $\quad$ S62 
General Information. Unless stated otherwise, reagents were obtained from commercial sources and used without further purification. All reactions were performed under an argon or nitrogen atmosphere in anhydrous solvents, which were dried prior to use following standard procedures. Reactions were monitored by thin-layer chromatography on $0.25 \mathrm{~mm}$ E. Merck silica gel plates (60F-254) using $7 \%$ ethanolic phosphomolybdic acid as developing agent. Merck silica gel 60 (particle size 0.040-0.063 mm, 230-400 mesh) was employed for flash chromatography. Melting points are uncorrected. IR spectra were recorded as films on $\mathrm{KBr}$ plates. ${ }^{1} \mathrm{H}$ NMR spectra were obtained in $\mathrm{CDCl}_{3}$ at $400 \mathrm{MHz}(\mathrm{Bruker}$ DPX-400) or $500 \mathrm{MHz}$ (Varian-Unity INOVA-500). ${ }^{13} \mathrm{C}$ NMR spectra were obtained at $100 \mathrm{MHz}$ or $125 \mathrm{MHz}$. Chemical shifts were reported in $\delta$ (ppm) using solvent resonance as the internal reference. High resolution mass spectra (HRMS) were obtained on a TOF MS instrument with an EI source. 
The comparison table for the ${ }^{1}$ H NMR data of natural (+)-parimycin, synthetic ( \pm )-1, and (-)-1

\begin{tabular}{|l|l|l|}
\hline Natural (+)-parimycin $\left(\mathrm{CDCl}_{3}\right) \delta$ & $\begin{array}{l}\text { Synthetic }( \pm) \text {-parimycin }(\mathbf{1})(500 \\
\left.\mathrm{MHz}, \mathrm{CDCl}_{3}\right) \delta(\mathrm{ppm})\end{array}$ & $\begin{array}{l}\text { Synthetic (-)-parimycin (1) (400 } \\
\left.\mathrm{MHz}, \mathrm{CDCl}_{3}\right) \delta(\mathrm{ppm})\end{array}$ \\
\hline $14.68(\mathrm{~s}, 1 \mathrm{H})$ & $14.71(\mathrm{~s}, 1 \mathrm{H})$ & $14.72(\mathrm{~s}, 1 \mathrm{H})$ \\
\hline $13.36(\mathrm{~s}, 1 \mathrm{H})$ & $13.38(\mathrm{~s}, 1 \mathrm{H})$ & $8.16(\mathrm{~s}, 1 \mathrm{H})$ \\
\hline $8.12(\mathrm{brs}, 1 \mathrm{H})$ & $8.14(\mathrm{~s}, 1 \mathrm{H})$ & $6.54(\mathrm{~s}, 1 \mathrm{H})$ \\
\hline $6.53(\mathrm{~s}, 1 \mathrm{H})$ & $6.53(\mathrm{~s}, 1 \mathrm{H})$ & $3.13(\mathrm{~s}, 4 \mathrm{H})$ \\
\hline $3.10(\mathrm{~s}, 4 \mathrm{H})$ & $3.13(\mathrm{~s}, 4 \mathrm{H})$ & $3.03(\mathrm{~s}, 3 \mathrm{H})$ \\
\hline $2.98(\mathrm{brs}, 3 \mathrm{H})$ & $3.01(\mathrm{~s}, 3 \mathrm{H})$ & $2.10-2.00(\mathrm{~m}, 1 \mathrm{H})$ \\
\hline $2.05(\mathrm{~m}, 1 \mathrm{H})$ & $2.10-2.00(\mathrm{~m}, 1 \mathrm{H})$ & $2.00-1.90(\mathrm{~m}, 1 \mathrm{H})$ \\
\hline $1.92(\mathrm{~m}, 1 \mathrm{H})$ & $2.00-1.90(\mathrm{~m}, 1 \mathrm{H})$ & $1.64(\mathrm{~s}, 3 \mathrm{H})$ \\
\hline $1.61(\mathrm{~s}, 3 \mathrm{H})$ & $1.64(\mathrm{~s}, 3 \mathrm{H})$ & $0.95(\mathrm{t}, J=7.6 \mathrm{~Hz}, 3 \mathrm{H})$ \\
\hline $0.93(\mathrm{t}, J=6.8 \mathrm{~Hz}, 3 \mathrm{H})$ & $0.95(\mathrm{t}, J=7.5 \mathrm{~Hz}, 3 \mathrm{H})$ & \\
\hline
\end{tabular}


The comparison table for the ${ }^{13} \mathrm{C}$ NMR data of natural (+)-parimycin and synthetic $( \pm)-1$

\begin{tabular}{|c|c|}
\hline Natural (+)-parimycin $\left(\mathrm{CD}_{3} \mathrm{OD} / \mathrm{CDCl}_{3}\right) \delta(\mathrm{ppm})$ & Synthetic $( \pm)-\mathbf{1}\left(125 \mathrm{MHz}, \mathrm{CDCl}_{3}\right) \delta(\mathrm{ppm})$ \\
\hline $201.6(\mathrm{C})$ & $201.5(\mathrm{C})$ \\
\hline $201.0(\mathrm{C})$ & $201.0(\mathrm{C})$ \\
\hline $180.3(\mathrm{C})$ & $179.5(\mathrm{C})$ \\
\hline $172.7(\mathrm{C})$ & $170.5(\mathrm{C})$ \\
\hline $156.7(\mathrm{C})$ & $157.1(\mathrm{C})$ \\
\hline $156.5(\mathrm{C})$ & $156.5(\mathrm{C})$ \\
\hline $152.0(\mathrm{C})$ & $152.7(\mathrm{C})$ \\
\hline $142.6(\mathrm{C})$ & $143.0(\mathrm{C})$ \\
\hline $132.4(\mathrm{C})$ & $132.6(\mathrm{C})$ \\
\hline $122.5(\mathrm{C})$ & $122.3 \times 2(\mathrm{C}, \mathrm{CH})$ \\
\hline $122.1(\mathrm{CH})$ & \\
\hline $117.4(\mathrm{C})$ & $117.6(\mathrm{C})$ \\
\hline $110.0(\mathrm{C})$ & $110.1(\mathrm{C})$ \\
\hline $109.5(\mathrm{CH})$ & $110.0(\mathrm{CH})$ \\
\hline $108.5(\mathrm{C})$ & $108.7(\mathrm{C})$ \\
\hline $73.6(\mathrm{C})$ & $74.1(\mathrm{C})$ \\
\hline $36.0\left(\mathrm{CH}_{2}\right)$ & $36.1\left(\mathrm{CH}_{2}\right)$ \\
\hline $35.8\left(\mathrm{CH}_{2}\right)$ & $35.9\left(\mathrm{CH}_{2}\right)$ \\
\hline $33.0\left(\mathrm{CH}_{2}\right)$ & $33.8\left(\mathrm{CH}_{2}\right)$ \\
\hline $25.7\left(\mathrm{CH}_{3}\right)$ & $25.7\left(\mathrm{CH}_{3}\right)$ \\
\hline $23.7\left(\mathrm{CH}_{3}\right)$ & $23.9\left(\mathrm{CH}_{3}\right)$ \\
\hline $7.7\left(\mathrm{CH}_{3}\right)$ & $8.1\left(\mathrm{CH}_{3}\right)$ \\
\hline
\end{tabular}


<smiles>COc1ccc(O)c2c1C(=O)CCC2=O</smiles>

6

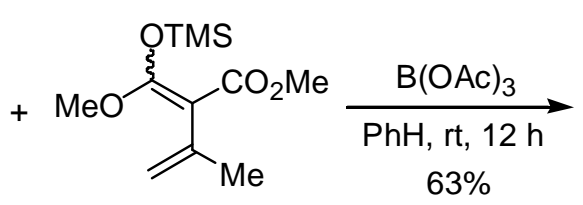

7<smiles>COc1ccc(O)c2c1C(=O)c1cc(C)c(C(C)=O)c(O)c1C2=O</smiles>

5

Methyl 1,8-dihydroxy-5-methoxy-3-methylanthraquinone-2-carboxylate (5). A mixture of $O$ methylnaphthazarin $(6)(500 \mathrm{mg}, 2.45 \mathrm{mmol})$ and $\mathrm{B}(\mathrm{OAc})_{3}(506 \mathrm{mg}, 2.69 \mathrm{mmol})$ in benzene $(30 \mathrm{~mL})$ was stirred at room temperature for $10 \mathrm{~min}$. A solution of 7 (800 $\mathrm{mg}, 3.27 \mathrm{mmol})$ in benzene $(10 \mathrm{~mL})$ was added to the reaction mixture and stirred for another $12 \mathrm{~h}$. The solvent was evaporated in vacuo to give a residue. The crude product was purified by column chromatography on silica gel $\left(\mathrm{CH}_{2} \mathrm{Cl}_{2}\right)$ to afford $5(528 \mathrm{mg}, 63 \%)$ as a red solid. Analytically pure 5 was obtained by crystallization from $\mathrm{CH}_{2} \mathrm{Cl}_{2}-$ hexane: Mp 213-214 ${ }^{\circ} \mathrm{C}$; IR (neat) v 2954, 2851, 1728, 1664, 1627, 1579, 1439, 1224, 1147, 1015, 932, 831, $775 \mathrm{~cm}^{-1} ;{ }^{1} \mathrm{H}$ NMR (400 MHz, $\left.\mathrm{CDCl}_{3}\right) \delta 12.31(\mathrm{~s}, 1 \mathrm{H}), 12.29(\mathrm{~s}, 1 \mathrm{H}), 7.63(\mathrm{~s}, 1 \mathrm{H}), 7.42(\mathrm{~d}, J=9.4$ $\mathrm{Hz}, 1 \mathrm{H}), 7.32(\mathrm{~d}, J=9.4 \mathrm{~Hz}, 1 \mathrm{H}), 4.01(\mathrm{~s}, 3 \mathrm{H}), 3.99(\mathrm{~s}, 3 \mathrm{H}), 2.45(\mathrm{~s}, 3 \mathrm{H}) ;{ }^{13} \mathrm{C} \mathrm{NMR}\left(100 \mathrm{MHz}, \mathrm{CDCl}_{3}\right)$ $\delta 192.1(\mathrm{C}), 180.4(\mathrm{C}), 166.6(\mathrm{C}), 159.2$ (C), 157.4 (C), 154.8 (C), 146.5 (C), 135.0 (C), 127.7 (C), $127.0(\mathrm{CH}), 124.2(\mathrm{CH}), 121.1(\mathrm{CH}), 118.8(\mathrm{C}), 115.5(\mathrm{C}), 113.7(\mathrm{C}), 57.0\left(\mathrm{CH}_{3}\right), 52.7\left(\mathrm{CH}_{3}\right), 20.6$ $\left(\mathrm{CH}_{3}\right)$; MS (EI) m/z (\% base peak) $342\left(\mathrm{M}^{+}, 59\right), 310$ (100), 295 (90), 281 (9), 267 (10), 253 (8), 239 (6), 211 (7), 197 (3), 183 (5), 168 (2), 155 (11), 139 (5), 127 (6), 97 (3), 83 (3), 71(4), 58 (4); HRMS (EI) calcd for $\mathrm{C}_{18} \mathrm{H}_{14} \mathrm{O}_{7} 342.0740$, Found 342.0741.<smiles>COc1ccc(O)c2c1C(=O)c1cc(C)c(O)c(c1O)C2=O</smiles> 
Methyl 1,5,8-trimethoxy-3-methylanthraquinone-2-carboxylate (9). To a stirred solution of 5 (260 $\mathrm{mg}, 0.76 \mathrm{mmol})$ in $\mathrm{DMF}(5 \mathrm{~mL})$ at $0{ }^{\circ} \mathrm{C}$ was added $\mathrm{Cs}_{2} \mathrm{CO}_{3}(1.00 \mathrm{~g}, 3.07 \mathrm{mmol})$ and methyl iodide $(0.50$ $\mathrm{mL}, 8.02 \mathrm{mmol})$. The mixture was then stirred at room temperature for $24 \mathrm{~h}$. The reaction mixture was quenched with $1 \mathrm{M} \mathrm{HCl}$ and diluted with water. The aqueous phase was extracted with $\mathrm{CH}_{2} \mathrm{Cl}_{2}$ and then washed with brine, dried over $\mathrm{MgSO}_{4}$, filtered and concentrated. The residue was purified by gradient silica-gel column chromatography $\left(\mathrm{CH}_{2} \mathrm{Cl}_{2} / \mathrm{EtOAc}=1: 0\right.$ to $\left.9: 1\right)$ to give $9(258 \mathrm{mg}, 92 \%)$ as a yellow solid. Analytically pure 9 was obtained by crystallization from $\mathrm{CH}_{2} \mathrm{Cl}_{2}-\mathrm{EtOAc}$ : Mp 266-268 ${ }^{\circ} \mathrm{C}$; IR (neat) $v$ 2948, 2844, 1733, 1670, 1279, 1225, 996, $820 \mathrm{~cm}^{-1} ;{ }^{1} \mathrm{H}$ NMR (400 MHz, $\left.\mathrm{CDCl}_{3}\right) \delta 7.77(\mathrm{~s}, 1 \mathrm{H})$, $7.32(\mathrm{~d}, J=9.6 \mathrm{~Hz}, 1 \mathrm{H}), 7.27(\mathrm{~d}, J=9.6 \mathrm{~Hz}, 1 \mathrm{H}), 4.00(\mathrm{~s}, 3 \mathrm{H}), 3.99(\mathrm{~s}, 3 \mathrm{H}), 3.98(\mathrm{~s}, 3 \mathrm{H}), 3.97(\mathrm{~s}, 3 \mathrm{H})$, $2.40(\mathrm{~s}, 3 \mathrm{H}) ;{ }^{13} \mathrm{C}$ NMR $\left(100 \mathrm{MHz}, \mathrm{CDCl}_{3}\right) \delta 183.0$ (C), 182.4 (C), 167.4 (C), 156.4 (C), $153.4(\mathrm{C})$, 152.9 (C), 141.7 (C), 136.7 (C), 134.8 (C), 125.3 (C), 125.2 (C), $123.7(\mathrm{CH}), 122.3(\mathrm{C}), 120.4(\mathrm{CH})$, $118.7(\mathrm{CH}), 63.8\left(\mathrm{CH}_{3}\right), 57.2\left(\mathrm{CH}_{3}\right), 56.9\left(\mathrm{CH}_{3}\right), 52.5\left(\mathrm{CH}_{3}\right), 19.7\left(\mathrm{CH}_{3}\right)$; MS (EI) $m / z(\%$ base peak) $370\left(\mathrm{M}^{+}, 7\right), 355$ (4), 338 (5), 325 (15), 219 (5), 187 (22), 159 (21), 140 (100), 112 (66), 109 (55), 82 (100), 67 (50), 59 (41); HRMS (EI) calcd for $\mathrm{C}_{20} \mathrm{H}_{18} \mathrm{O}_{7}$ 370.1053, Found 370.1052.

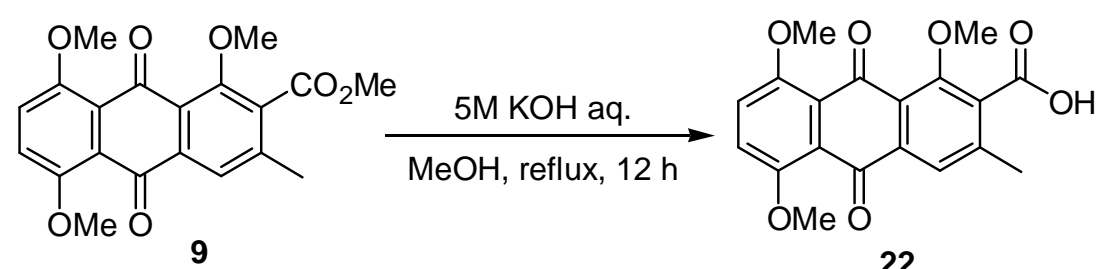

1,5,8-Trimethoxy-3-methylanthraquinone-2-carboxylic acid (22). To a stirred solution of 9 (134 mg, $0.36 \mathrm{mmol})$ in methanol $(20 \mathrm{~mL})$ was added $5 \mathrm{M} \mathrm{KOH}(20 \mathrm{~mL})$. The mixture was heated to reflux for 12 h. After cooled to room temperature, the mixture was acidified with $1 \mathrm{M} \mathrm{HCl}$. The solvent was evaporated in vacuo to provide $22(121 \mathrm{mg})$ as an orange-red solid, which was used in the next step without further purification. Analytically pure 22 was obtained by crystallization from $\mathrm{MeOH}$ : $\mathrm{Mp}>235$ ${ }^{\circ} \mathrm{C}$ (dec.); IR (neat) v 3500-2500, 2969, 1663, 1562, 1280, 1068, 988, $817 \mathrm{~cm}^{-1} ;{ }^{1} \mathrm{H}$ NMR (400 MHz, 
$\left.\mathrm{CD}_{3} \mathrm{OD}\right) \delta 7.67(\mathrm{~s}, 1 \mathrm{H}), 7.45(\mathrm{~d}, J=9.5 \mathrm{~Hz}, 1 \mathrm{H}), 7.40(\mathrm{~d}, J=9.5 \mathrm{~Hz}, 1 \mathrm{H}), 3.97(\mathrm{~s}, 3 \mathrm{H}), 3.91(\mathrm{~s}, 3 \mathrm{H})$, $3.90(\mathrm{~s}, 3 \mathrm{H}), 2.46(\mathrm{~s}, 3 \mathrm{H}) ;{ }^{13} \mathrm{C} \mathrm{NMR}\left(100 \mathrm{MHz}, \mathrm{CD}_{3} \mathrm{OD}\right) \delta 185.2,184.6,174.4,155.7,154.7,154.2$, $144.7,141.7,135.5,126.6,126.0,124.5,122.9,122.2,120.7,63.5,57.4,57.2,20.0 ; \mathrm{MS}(\mathrm{EI}) \mathrm{m} / z(\%$ base peak) $356\left(\mathrm{M}^{+}, 7\right), 355$ (18), 339 (33), 325 (100), 298 (25), 295 (21), 281 (20), 267 (14), 253 (14), 239 (6), 181 (8), 165 (11), 149 (17), 125 (20), 111 (34), 97 (49), 83 (51), 71 (65), 57 (63); HRMS (EI) calcd for $\mathrm{C}_{19} \mathrm{H}_{16} \mathrm{O}_{7} 356.0896$, Found 356.0896.<smiles>COc1ccc(OC)c2c1C(=O)c1cc(C)c(C(=O)O)c(OC)c1C2=O</smiles>

22

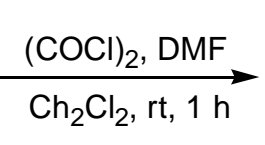<smiles>COc1ccc(OC)c2c1C(=O)c1cc(C)c(C(=O)Cl)c(OC)c1C2=O</smiles>

10

1,5,8-Trimethoxy-3-methylanthraquinone-2-carbonyl chloride (10). To a stirred solution of crude acid $22(121 \mathrm{mg}, 0.34 \mathrm{mmol})$ in dry $\mathrm{CH}_{2} \mathrm{Cl}_{2}(20 \mathrm{~mL})$ was added oxalyl chloride $(0.24 \mathrm{~mL}, 2.82 \mathrm{mmol})$ and 1 drop DMF under argon atmosphere. The mixture was stirred at room temperature for $1 \mathrm{~h}$. The solvent was removed in vacuo to give $\mathbf{1 0}(120 \mathrm{mg})$ as an orange-yellow solid, which was used in the next step without further purification. Analytically pure $\mathbf{1 0}$ was obtained by silica-gel column chromatography (hexane/EtOAc = 2:1): $\mathrm{Mp}>175^{\circ} \mathrm{C}(\mathrm{dec}$.$) ; IR (neat) v 2923, 2844, 1787, 1668, 1588,$ 1408, 1327, 1228, 1000, 925, 885, $817 \mathrm{~cm}^{-1} ;{ }^{1} \mathrm{H}$ NMR (400 MHz, $\left.\mathrm{CDCl}_{3}\right) \delta 7.79(\mathrm{~s}, 1 \mathrm{H}), 7.33(\mathrm{~d}, J=$ $9.3 \mathrm{~Hz}, 1 \mathrm{H}), 7.27(\mathrm{~d}, J=9.3 \mathrm{~Hz}, 1 \mathrm{H}), 4.04$ (s, 3H), 3.98 (s, 3H), 3.97 (s, 3H), $2.48(\mathrm{~s}, 3 \mathrm{H}) ;{ }^{13} \mathrm{C}$ NMR $\left(100 \mathrm{MHz}, \mathrm{CDCl}_{3}\right) \delta 182.4(\mathrm{C}), 182.2(\mathrm{C}), 167.6(\mathrm{C}), 155.0(\mathrm{C}), 153.5$ (C), $152.9(\mathrm{C}), 139.6(\mathrm{C}), 138.3$ (C), $137.3(\mathrm{C}), 125.2(\mathrm{C}), 125.1(\mathrm{C}), 123.9(\mathrm{CH}), 122.1(\mathrm{C}), 120.5(\mathrm{CH}), 119.0(\mathrm{CH}), 63.9\left(\mathrm{CH}_{3}\right), 57.2$ $\left(\mathrm{CH}_{3}\right), 56.9\left(\mathrm{CH}_{3}\right), 19.4\left(\mathrm{CH}_{3}\right)$; MS (EI) $m / z$ (\% base peak) $376\left(\mathrm{M}^{+}+2,5\right), 374\left(\mathrm{M}^{+}, 15\right), 339(81), 325$ (9), 309 (9), 295 (8), 279 (14), 261 (14), 253 (8), 239 (6), 223 (5), 197 (5), 183 (6), 167 (34), 149 (100), 125 (17), 111 (31), 85 (59), 71 (94), 57 (81); HRMS (EI) calcd for $\mathrm{C}_{19} \mathrm{H}_{15} \mathrm{O}_{6}{ }^{35} \mathrm{Cl} 374.0557$, Found 374.0559. 


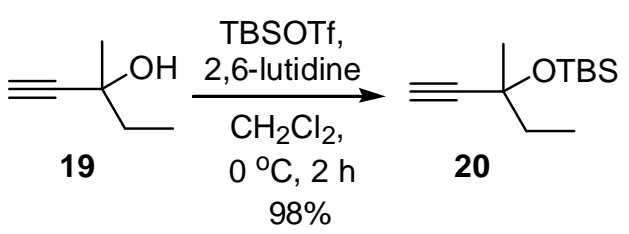

tert-Butyldimethyl((3-methylpent-1-yn-3-yl)oxy)silane (20). To a stirred solution of 3-methyl-1pentyn-3-ol (19) (200 mg, $2.04 \mathrm{mmol})$ in $\mathrm{CH}_{2} \mathrm{Cl}_{2}(6 \mathrm{~mL})$ at $0{ }^{\circ} \mathrm{C}$ was added 2,6-lutidine $(0.56 \mathrm{~mL}, 4.83$ mmol) and TBSOTf $(0.56 \mathrm{~mL}, 2.45 \mathrm{mmol})$. The reaction mixture was stirred at $0{ }^{\circ} \mathrm{C}$ for $2 \mathrm{~h}$ and then quenched with dry methanol $(5 \mathrm{~mL})$ and stirred for another $30 \mathrm{~min}$. The solvent was removed in vacuo to give a residue. The crude product was purified by silica-gel column chromatography (hexane) to give 20 (424 mg, 98\%) as a colorless liquid. IR (neat) $v$ 3311, 2958, 2933, 2858, 1473, 1369, 1253, 1167 , 1064, 1007, 838, $776 \mathrm{~cm}^{-1} ;{ }^{1} \mathrm{H}$ NMR $\left(400 \mathrm{MHz}, \mathrm{CDCl}_{3}\right) \delta 2.39(\mathrm{~s}, 1 \mathrm{H}), 1.68-1.60(\mathrm{~m}, 2 \mathrm{H}), 1.43(\mathrm{~s}, 3 \mathrm{H})$, $0.99(\mathrm{t}, J=7.3 \mathrm{~Hz}, 3 \mathrm{H}), 0.87(\mathrm{~s}, 9 \mathrm{H}), 0.17(\mathrm{~s}, 6 \mathrm{H}),{ }^{13} \mathrm{C} \mathrm{NMR}\left(100 \mathrm{MHz}, \mathrm{CDCl}_{3}\right) \delta 88.6,72.1,69.8,38.4$, 30.8, 26.1, 18.4, 9.2, -2.5, -2.8; MS (EI) $\mathrm{m} / z$ (\% base peak) $212\left(\mathrm{M}^{+}, 1\right), 197$ (7), 183 (8), 169 (10), 155 (13), 141 (14), 125 (23), 111 (40), 97 (63), 85 (79), 71 (100), 57 (89); HRMS (EI) calcd for $\mathrm{C}_{12} \mathrm{H}_{24} \mathrm{OSi}$ 212.1596, Found 212.1599.

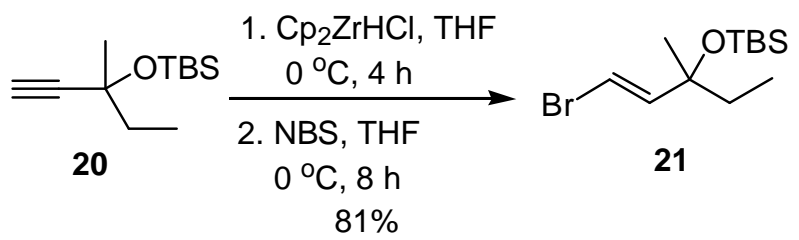

(E)-((1-Bromo-3-methylpent-1-en-3-yl)oxy)tert-butyldimethylsilane (21). To a stirred solution of zirconocene chloride hydride, which was prepared from zirconocene dichloride (1.56 g, $5.34 \mathrm{mmol})$ in dry THF $(30 \mathrm{~mL})$ and DIBAL-H $(5.6 \mathrm{~mL}, 1.00 \mathrm{M}$ in hexane, $5.60 \mathrm{mmol})$ at $0{ }^{\circ} \mathrm{C}$ for $1 \mathrm{~h}$, was added 20 
$(1.05 \mathrm{~g}, 4.94 \mathrm{mmol})$ and stirred at $0{ }^{\circ} \mathrm{C}$ for $4 \mathrm{~h}$. NBS $(1.10 \mathrm{~g}, 6.18 \mathrm{mmol})$ was added to the reaction mixture at $0{ }^{\circ} \mathrm{C}$ and stirred for another $8 \mathrm{~h}$. The reaction mixture was added hexanes $(30 \mathrm{~mL})$ and then filtered. The filtrate was wished with saturated aqueous $\mathrm{NH}_{4} \mathrm{Cl}$, brine, dried over $\mathrm{MgSO}_{4}$, filtered and concentrated. The residue was purified by chromatography on alumina (hexane) to give 21 (1.18 $\mathrm{g}, 81 \%$ ) as a colorless liquid. IR (neat) $v 3084,2958,2932,2857,1617,1463,1255,1064,1005,836,773 \mathrm{~cm}^{-1}$; ${ }^{1} \mathrm{H}$ NMR (400 MHz, $\left.\mathrm{CD}_{2} \mathrm{Cl}_{2}\right) \delta 6.23(\mathrm{~d}, J=13.4 \mathrm{~Hz}, 1 \mathrm{H}), 6.19(\mathrm{~d}, J=13.4 \mathrm{~Hz}, 1 \mathrm{H}), 1.60-1.45(\mathrm{~m}, 2 \mathrm{H})$, $1.30(\mathrm{~s}, 3 \mathrm{H}), 0.90(\mathrm{~s}, 9 \mathrm{H}), 0.87(\mathrm{t}, J=7.5 \mathrm{~Hz}, 3 \mathrm{H}), 0.10(\mathrm{~s}, 3 \mathrm{H}), 0.09(\mathrm{~s}, 3 \mathrm{H}) ;{ }^{13} \mathrm{C}$ NMR $(100 \mathrm{MHz}$, $\left.\mathrm{CD}_{2} \mathrm{Cl}_{2}\right) \delta 144.5,104.3,76.8,35.8,26.8,25.4,17.9,7.8,-2.7,-2.8$; MS (EI) $m / z$ (\% base peak) $292\left(\mathrm{M}^{+}\right.$, 0.1), 263 (1), 246 (2), 231 (2), 221 (4), 189 (50), 147 (100), 133 (8), 113 (7), 83 (4), 73 (18); HRMS (EI) calcd for $\mathrm{C}_{12} \mathrm{H}_{25}{ }^{79} \mathrm{BrOSi} 292.0858$, Found 292.0857.<smiles>COc1ccc(OC)c2c1C(=O)c1cc(C)c(C(C)=O)c(OC)c1C2=O</smiles>

10

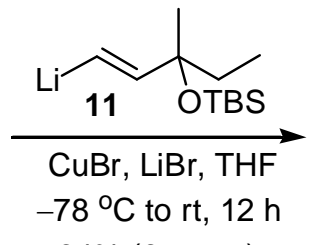

$64 \%$ (3 steps)<smiles>CCC(C)(/C=C/C(=O)c1c(C)cc2c(c1OC)-c1c(OC)ccc(OC)c1C2=O)C[Se]</smiles>

12

\section{(士)-2-[3-(tert-Butyldimethylsilyloxy)-3-methyl-1-pentenyl]-1,5,8-trimethoxy-3-}

methylanthraquinone (12). To a stirred solution of $11(0.85 \mathrm{mmol})$ in $\mathrm{THF}$ at $-78{ }^{\circ} \mathrm{C}$, which was prepared from $21(250 \mathrm{mg}, 0.85 \mathrm{mmol})$ in THF $(10 \mathrm{~mL})$ and tert-BuLi $(1.94 \mathrm{M}$ in pentane, $0.85 \mathrm{~mL}$, $1.65 \mathrm{mmol})$ at $-78{ }^{\circ} \mathrm{C}$ for $1 \mathrm{~h}$, was added a solution of $\mathrm{CuBr}(122 \mathrm{mg}, 0.85 \mathrm{mmol})$ and $\mathrm{LiBr}(148 \mathrm{mg}$, $1.70 \mathrm{mmol})$ in THF (5 mL) and this solution was then added a solution of crude acid chloride $\mathbf{1 0}$ (120 $\mathrm{mg}, 0.32 \mathrm{mmol})$ in THF ( $5 \mathrm{~mL})$. The reaction mixture was then allowed to warm to room temperature and stirred for $12 \mathrm{~h}$. The mixture was quenched with saturated aqueous $\mathrm{NH}_{4} \mathrm{Cl}$. The aqueous solution was extracted with $\mathrm{CH}_{2} \mathrm{Cl}_{2}$, and the combined extracts were washed with brine, dried over $\mathrm{MgSO}_{4}$, filtered and concentrated. The residue was purified by silica-gel chromatography (hexane/EtOAc $=3: 2$ ) 
to afford 12 (127 mg, 64\%, over 3 steps) as a yellow solid. Analytically pure 12 was obtained by crystallization from $\mathrm{CH}_{2} \mathrm{Cl}_{2}$-hexane: $\mathrm{Mp} 168-169{ }^{\circ} \mathrm{C}$; IR (neat) v 2929, 2856, 1667, 1588, 1466, 1276, 1226, 1058, 987, $837 \mathrm{~cm}^{-1} ;{ }^{1} \mathrm{H}$ NMR (400 MHz, $\left.\mathrm{CDCl}_{3}\right) \delta 7.76(\mathrm{~s}, 1 \mathrm{H}), 7.31(\mathrm{~d}, J=9.4 \mathrm{~Hz}, 1 \mathrm{H}), 7.26(\mathrm{~d}$, $J=9.4 \mathrm{~Hz}, 1 \mathrm{H}), 6.50(\mathrm{~d}, J=15.8 \mathrm{~Hz}, 1 \mathrm{H}), 6.42(\mathrm{~d}, J=15.8 \mathrm{~Hz}, 1 \mathrm{H}), 3.98(\mathrm{~s}, 3 \mathrm{H}), 3.96(\mathrm{~s}, 3 \mathrm{H}), 3.87(\mathrm{~s}$, 3H), $2.28(\mathrm{~s}, 3 \mathrm{H}), 1.60-1.50(\mathrm{~m}, 2 \mathrm{H}), 1.31(\mathrm{~s}, 3 \mathrm{H}), 0.88(\mathrm{~s}, 9 \mathrm{H}), 0.81(\mathrm{t}, J=7.4 \mathrm{~Hz}, 3 \mathrm{H}), 0.07(\mathrm{~s}, 3 \mathrm{H})$, $0.05(\mathrm{~s}, 3 \mathrm{H}) ;{ }^{13} \mathrm{C}$ NMR $\left(100 \mathrm{MHz}, \mathrm{CDCl}_{3}\right) \delta 196.8,183.2,182.7,158.5,156.3,153.4,153.0$,141.6, $140.4,136.3,128.7,125.3,125.2,123.9,122.5,120.3,118.7,75.7,63.6,57.2,56.9,35.8,27.4,25.9$, 19.5, 18.4, 8.3, -2.1, -2.2; MS (EI) $m / z$ (\% base peak) $554\left(\mathrm{M}^{+}+2,1\right) ; 552\left(\mathrm{M}^{+}, 0.3\right), 523(5), 495(100)$, 397 (5), 339 (79), 281 (3), 253 (3), 181 (1), 152 (3), 139 (2), 115 (1), 75 (25); HRMS (EI) calcd for $\mathrm{C}_{31} \mathrm{H}_{40} \mathrm{O}_{7} \mathrm{Si}$ 552.2543, Found 552.2546.

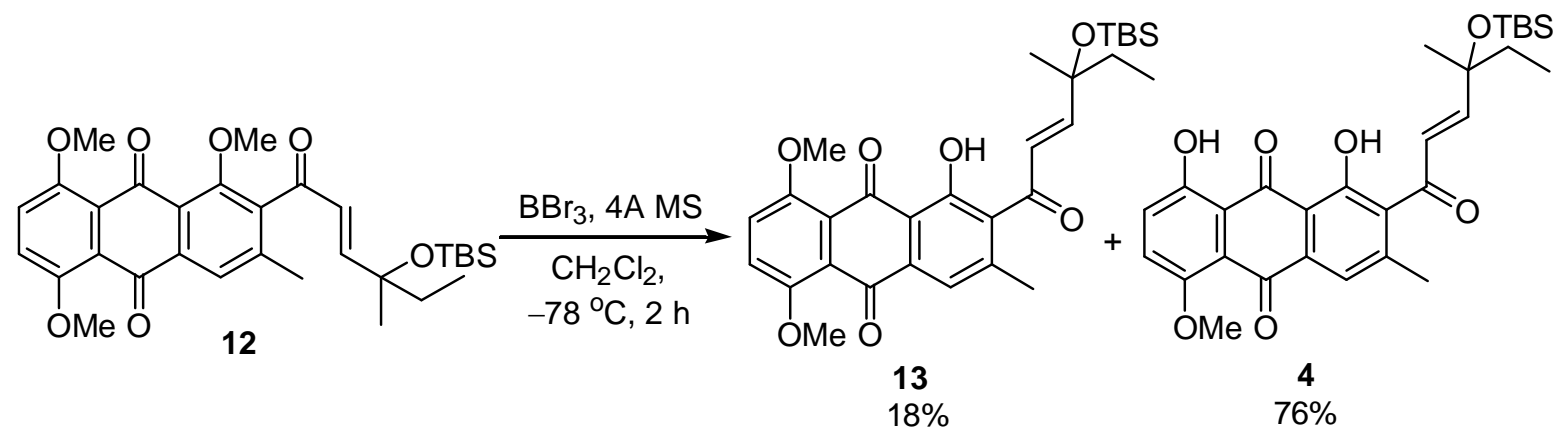

\section{(E)-2-(4-((tert-Butyldimethylsilyl)oxy)-4-methylhex-2-enoyl)-1-hydroxy-5,8-dimethoxy-3-}

methylanthraquinone (13) and (E)-2-(4-((tert-butyldimethylsilyl)oxy)-4-methylhex-2-enoyl)-1,8dihydroxy-5-methoxy-3-methylanthraquinone (4). To a stirred mixture of 12 (65 mg, $0.12 \mathrm{mmol})$ and dry molecular sieve $4 \AA(1.00 \mathrm{~g})$ in $\mathrm{CH}_{2} \mathrm{Cl}_{2}(13 \mathrm{~mL})$ at $-78{ }^{\circ} \mathrm{C}$ was added $\mathrm{BBr}_{3}\left(1 \mathrm{M}\right.$ in $\mathrm{CH}_{2} \mathrm{Cl}_{2}, 0.36 \mathrm{~mL}$, $0.36 \mathrm{mmol}$ ). After stirred at $-78{ }^{\circ} \mathrm{C}$ for $2 \mathrm{~h}$, the mixture was quenched with water. The aqueous phase was extracted with $\mathrm{CH}_{2} \mathrm{Cl}_{2}$. The combined extracts were washed with brine, dried over $\mathrm{MgSO}_{4}$, filtered and concentrated. The crude product was purified by gradient silica-gel chromatography (hexane/ $\mathrm{CH}_{2} \mathrm{Cl}_{2}=1: 3$ to $\left.0: 1\right)$ to give $\mathbf{1 3}(12 \mathrm{mg}, 18 \%$ ) as a red solid and $\mathbf{4}(46 \mathrm{mg}, 76 \%)$ as a red solid. 
Analytically pure 13 was obtained by crystallization from $\mathrm{CH}_{2} \mathrm{Cl}_{2}$-hexane: $\mathrm{Mp} 165-166{ }^{\circ} \mathrm{C}$; IR (neat) $v$ 2930, 2856, 1656, 1589, 1408, 1229, 1178, 1075, 836, $773 \mathrm{~cm}^{-1} ;{ }^{1} \mathrm{H}$ NMR (400 MHz, $\left.\mathrm{CDCl}_{3}\right) \delta 12.65$ (s, 1H), $7.94(\mathrm{~s}, 1 \mathrm{H}), 7.39(\mathrm{~d}, J=9.6 \mathrm{~Hz}, 1 \mathrm{H}), 7.27(\mathrm{~d}, J=9.6 \mathrm{~Hz}, 1 \mathrm{H}), 6.51(\mathrm{~d}, J=16.0 \mathrm{~Hz}, 1 \mathrm{H}), 6.41$ $(\mathrm{d}, J=16.0 \mathrm{~Hz}, 1 \mathrm{H}), 3.98(\mathrm{~s}, 3 \mathrm{H}), 3.88(\mathrm{~s}, 3 \mathrm{H}), 2.33(\mathrm{~s}, 3 \mathrm{H}), 1.85-1.55(\mathrm{~m}, 2 \mathrm{H}), 1.31(\mathrm{~s}, 3 \mathrm{H}), 0.88(\mathrm{~s}$, 9H), $0.81(\mathrm{t}, J=7.4 \mathrm{~Hz}, 3 \mathrm{H}), 0.07(\mathrm{~s}, 3 \mathrm{H}), 0.05(\mathrm{~s}, 3 \mathrm{H}) ;{ }^{13} \mathrm{C} \mathrm{NMR}\left(100 \mathrm{MHz}, \mathrm{CDCl}_{3}\right) \delta 196.4(\mathrm{C}), 188.3$ (C), $181.0(C), 158.6,157.4,156.7,153.4,142.4,141.8,134.0,128.4,125.6,125.0,124.2,124.1,121.0$, 115.6, 75.6, 63.4, 57.1, 35.7, 27.3, 25.8, 19.3, 18.3, 8.2, -2.2, -2.3; MS (EI) $m / z$ (\% base peak) $538\left(\mathrm{M}^{+}\right.$, 0.2), 523 (2), 509 (5), 482 (42), 481 (100), 449 (6), 407 (3), 379 (2), 311 (7), 233 (2), 139 (1), 95 (1), 75 (17), 73 (16); HRMS (EI) calcd for $\mathrm{C}_{30} \mathrm{H}_{38} \mathrm{O}_{7} \mathrm{Si}$ 538.2387, Found 538.2388. Analytically pure 4 was obtained by crystallization from $\mathrm{CH}_{2} \mathrm{Cl}_{2}$-hexane: $\mathrm{Mp} 158-159^{\circ} \mathrm{C}$; IR (neat) v 2929, 2854, 1723, 1660, 1619, 1232, 1066, $836 \mathrm{~cm}^{-1} ;{ }^{1} \mathrm{H}$ NMR $\left(400 \mathrm{MHz}, \mathrm{CDCl}_{3}\right) \delta 13.20(\mathrm{~s}, 1 \mathrm{H}), 13.09(\mathrm{~s}, 1 \mathrm{H}), 7.68(\mathrm{~s}, 1)$, $7.43(\mathrm{~d}, J=9.6 \mathrm{~Hz}, 1 \mathrm{H}), 7.37(\mathrm{~d}, J=9.6 \mathrm{~Hz}, 1 \mathrm{H}), 6.54-6.52(\mathrm{~m}, 2 \mathrm{H}), 4.05(\mathrm{~s}, 3 \mathrm{H}), 2.34(\mathrm{~s}, 3 \mathrm{H}), 1.62-$ $1.50(\mathrm{~m}, 2 \mathrm{H}), 1.34(\mathrm{~s}, 3 \mathrm{H}), 0.88(\mathrm{~s}, 9 \mathrm{H}), 0.83(\mathrm{t}, J=7.4 \mathrm{~Hz}, 1 \mathrm{H}), 0.08(\mathrm{~s}, 3 \mathrm{H}), 0.05(\mathrm{~s}, 3 \mathrm{H}) ;{ }^{13} \mathrm{C} \mathrm{NMR}$ (100 MHz, $\left.\mathrm{CDCl}_{3}\right) \delta 196.1(\mathrm{C}), 187.9(\mathrm{C}), 187.4(\mathrm{C}), 159.7$ (C), $158.3(\mathrm{CH}), 157.9$ (C), $155.0(\mathrm{C})$, $144.4(\mathrm{C}), 135.5(\mathrm{C}), 132.1(\mathrm{C}), 128.5(\mathrm{CH}), 127.6(\mathrm{CH}), 123.7(\mathrm{CH}), 120.2(\mathrm{CH}), 118.1(\mathrm{C}), 115.6(\mathrm{C})$, $115.1(\mathrm{C}), 75.7(\mathrm{C}), 57.0\left(\mathrm{CH}_{3}\right), 35.9\left(\mathrm{CH}_{2}\right), 27.3\left(\mathrm{CH}_{3}\right), 25.9\left(\mathrm{CH}_{3}\right), 19.9(\mathrm{C}), 18.3\left(\mathrm{CH}_{3}\right), 8.3\left(\mathrm{CH}_{3}\right),-$ $2.1\left(\mathrm{CH}_{3}\right),-2.2\left(\mathrm{CH}_{3}\right)$; MS (EI) $\mathrm{m} / z$ (\% base peak) $524\left(\mathrm{M}^{+}, 1\right), 481(17), 467$ (100), 449 (55), 377 (6), 363 (5), 311 (12), 282 (4), 212 (1), 187 (2), 129 (2), 73 (20); HRMS (EI) calcd for $\mathrm{C}_{29} \mathrm{H}_{36} \mathrm{O}_{7} \mathrm{Si} 524.2230$, Found 524.2232.
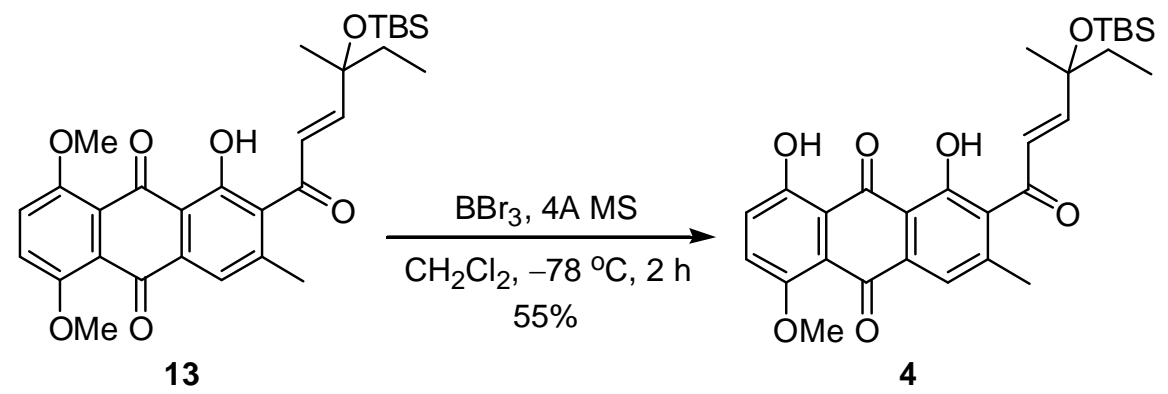


\section{(E)-2-(4-((tert-butyldimethylsilyl)oxy)-4-methylhex-2-enoyl)-1,8-dihydroxy-5-methoxy-3-}

methylanthraquinone (4). To a stirred mixture of $\mathbf{1 3}$ (30 mg, $0.056 \mathrm{mmol})$ and dry molecular sieve $4 \AA$ (400 mg) in $\mathrm{CH}_{2} \mathrm{Cl}_{2}(6 \mathrm{~mL})$ at $-78{ }^{\circ} \mathrm{C}$ was added $\mathrm{BBr}_{3}\left(1 \mathrm{M}\right.$ in $\left.\mathrm{CH}_{2} \mathrm{Cl}_{2}, 0.11 \mathrm{~mL}, 0.11 \mathrm{mmol}\right)$. After stirred at $-78{ }^{\circ} \mathrm{C}$ for $2 \mathrm{~h}$, the mixture was quenched with water. The aqueous phase was extracted with $\mathrm{CH}_{2} \mathrm{Cl}_{2}$. The combined extracts were washed with brine, dried over $\mathrm{MgSO}_{4}$, filtered and concentrated. The crude product was purified by gradient silica-gel chromatography (hexane $/ \mathrm{CH}_{2} \mathrm{Cl}_{2}=1: 3$ to $0: 1$ ) to give $4(16 \mathrm{mg}, 55 \%)$ as a red solid.

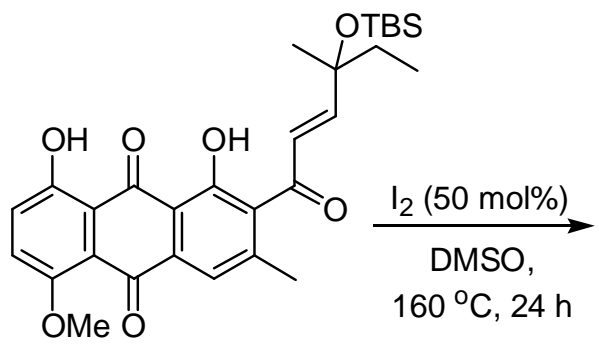

4<smiles>CCC(C)(O)c1cc(=O)c2c(C)cc3c(c2o1)C(=O)c1c(O)ccc(OC)c1C3=O</smiles>

14

\section{(士)-2-(1-Hydroxy-1-methylpropyl)-11-hydroxyl-8-methoxy-5-methylanthra[1,2-b]pyran-4,7,12-}

trione (14). To a stirred solution of $4(15 \mathrm{mg}, 0.029 \mathrm{mmol})$ in dry DMSO $(2 \mathrm{~mL})$ was added $\mathrm{I}_{2}(4 \mathrm{mg}$, $0.015 \mathrm{mmol}$ ) at room temperature. The reaction mixture was heated to $160{ }^{\circ} \mathrm{C}$ for $24 \mathrm{~h}$. After cooled to room temperature, the reaction mixture was diluted with $\mathrm{CH}_{2} \mathrm{Cl}_{2}$. The reaction mixture was washed with water and brine, dried over $\mathrm{MgSO}_{4}$, filtered and concentrated to give 14 (7.4 mg) as a red solid, which was found to be easily decomposed during the purification process. Thus, the crude product was used in the next step without further purification. 
<smiles>CCC(C)(O)c1cc(=O)c2c(C)cc3c(c2o1)C(=O)c1c(O)ccc(OC)c1C3=O</smiles>

14<smiles>CCC(C)(O)c1cc(=O)c2c(C)cc3c(c2o1)C(=O)c1c(O)ccc(O)c1C3=O</smiles>

3

To a stirred solution of crude $14(7.4 \mathrm{mg}, 0.018 \mathrm{mmol})$ in $\mathrm{CH}_{2} \mathrm{Cl}_{2}(3 \mathrm{~mL})$ at $-78{ }^{\circ} \mathrm{C}$ was added $\mathrm{BBr}_{3}(1$ $\mathrm{M}$ in $\mathrm{CH}_{2} \mathrm{Cl}_{2}, 0.14 \mathrm{~mL}, 0.14 \mathrm{mmol}$ ). The mixture was allowed to warm to $-50{ }^{\circ} \mathrm{C}$ and stirred at this temperature for $3 \mathrm{~h}$. The mixture was quenched with water and extracted with $\mathrm{CH}_{2} \mathrm{Cl}_{2}$. The combined extracts were washed with brine, dried over $\mathrm{MgSO}_{4}$, filtered and concentrated to give $\mathbf{3}(5.5 \mathrm{mg})$ as a red solid, which was found to be easily decomposed during the purification process. Thus, the crude product was used in the next step without further purification.

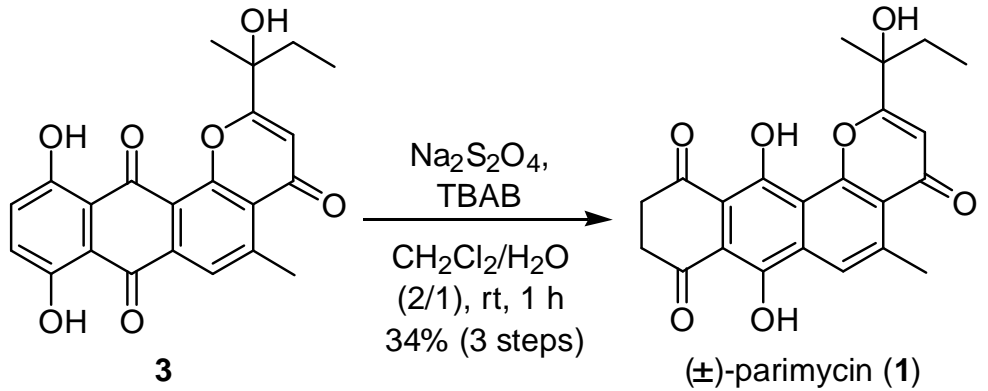

( \pm )-Parimycin (1). To a stirred mixture of sodium dithionite $(22 \mathrm{mg}, 0.126 \mathrm{mmol})$, and TBAB (2 mg, $0.006 \mathrm{mmol})$ in $\mathrm{H}_{2} \mathrm{O}(2 \mathrm{~mL})$ was added a solution of crude $3(5.5 \mathrm{mg}, 0.014 \mathrm{mmol})$ in $\mathrm{CH}_{2} \mathrm{Cl}_{2}(3.5 \mathrm{~mL})$ at room temperature. The reaction mixture was stirred at room temperature for $1 \mathrm{~h}$ and then quenched with water. The aqueous phase was extracted with $\mathrm{CH}_{2} \mathrm{Cl}_{2}$. The combined extracts were washed with brine, dried over $\mathrm{MgSO}_{4}$, filtered and concentrated. The crude product was purified by silica-gel chromatography $\left(\mathrm{CH}_{2} \mathrm{Cl}_{2} / \mathrm{EtOAc}=9: 1\right)$ to give $1(3.9 \mathrm{mg}, 34 \%$ over 3 steps $)$ as a yellow solid: $\mathrm{Mp}>$ 
$120{ }^{\circ} \mathrm{C}$ (dec.); IR (neat) $v 3405,2963,1650,1412,1318,1260,1184,1095,1020,800 \mathrm{~cm}^{-1} ;{ }^{1} \mathrm{H}$ NMR $\left(500 \mathrm{MHz}, \mathrm{CDCl}_{3}\right) \delta 14.71(\mathrm{~s}, 1 \mathrm{H}), 13.38(\mathrm{~s}, 1 \mathrm{H}), 8.14(\mathrm{~s}, 1 \mathrm{H}), 6.53(\mathrm{~s}, 1 \mathrm{H}), 3.13(\mathrm{~s}, 4 \mathrm{H}), 3.01(\mathrm{~s}, 3 \mathrm{H})$, 2.10-2.00 (m, 1H), 2.00-1.90 (m, 1H), $1.64(\mathrm{~s}, 3 \mathrm{H}), 0.95(\mathrm{t}, J=7.5 \mathrm{~Hz}, 3 \mathrm{H}) ;{ }^{13} \mathrm{C}$ NMR $(125 \mathrm{MHz}$, $\left.\mathrm{CDCl}_{3}\right) \delta 201.5,201.0,179.5,170.5,157.1,156.5,152.7,143.0,132.6,122.3 * 2,117.6,110.1,110.0$ 108.7, 74.1, 36.1, 35.9, 33.8, 25.7, 23.9, 8.1; MS (EI) $\mathrm{m} / z$ (\% base peak) $396\left(\mathrm{M}^{+}, 41\right), 378(100), 334$ (5), 298 (27), 270 (5), 214 (6), 151 (5), 95 (3); HRMS (EI) calcd for $\mathrm{C}_{22} \mathrm{H}_{20} \mathrm{O}_{7}$ 396.1209, Found 396.1214 .

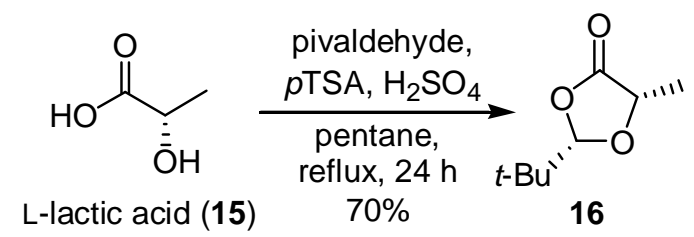

(2S,5S)-2-(tert-Butyl)-5-methyl-1,3-dioxolan-4-one (16). A mixture of L-lactic acid (4.5 g, $50 \mathrm{mmol})$, pivaldehyde ( $8.6 \mathrm{~g}, 100 \mathrm{mmol}), p$-toluenesulfonic acid monohydrate $(100 \mathrm{mg}, 0.53 \mathrm{mmol})$, and $1 \mathrm{drop}$ conc. $\mathrm{H}_{2} \mathrm{SO}_{4}$ in pentane $(20 \mathrm{~mL})$ was heated to reflux for $24 \mathrm{~h}$. After cooled to room temperature, the reaction mixture was washed with water, dried over $\mathrm{MgSO}_{4}$, filtered, and concentrated to give a yellow liquid $(7.3 \mathrm{~g}$, cis:trans $=6.7: 1)$. The residue was purified by crystallization from hexane at $-80{ }^{\circ} \mathrm{C}$ to give pure $16(5.5 \mathrm{~g}, 70 \%)$ as a colorless liquid. $[\alpha]^{25}{ }_{\mathrm{D}}=+45.9\left(c 1.00, \mathrm{CHCl}_{3}\right)\left[\right.$ lit. $^{1}[\alpha]^{20}{ }_{\mathrm{D}}=+44.8(c$ 1.83, $\left.\mathrm{CHCl}_{3}\right)$ ]; IR (neat) $v 2968,2875,1806,1486,1409,1301,1200,972 \mathrm{~cm}^{-1} ;{ }^{1} \mathrm{H}$ NMR (400 MHz, $\left.\mathrm{CDCl}_{3}\right) \delta 5.14(\mathrm{~s}, 1 \mathrm{H}), 4.35(\mathrm{q}, J=6.9 \mathrm{~Hz}, 1 \mathrm{H}), 1.49(\mathrm{~d}, J=6.9 \mathrm{~Hz}, 3 \mathrm{H}), 0.97(\mathrm{~s}, 9 \mathrm{H}) ;{ }^{13} \mathrm{C} \mathrm{NMR}(100$ $\left.\mathrm{MHz}, \mathrm{CDCl}_{3}\right) \delta 174.1,109.3,71.4,34.2,23.3,16.2$; MS (EI) $m / z$ (\% base peak) $158\left(\mathrm{M}^{+}, 2\right), 129$ (3), 113 (8), 101 (56), 87 (43), 85 (18), 73 (72), 57 (100); HRMS (EI) calcd for $\mathrm{C}_{8} \mathrm{H}_{14} \mathrm{O}_{3}$ 158.0943, Found 158.0941. 


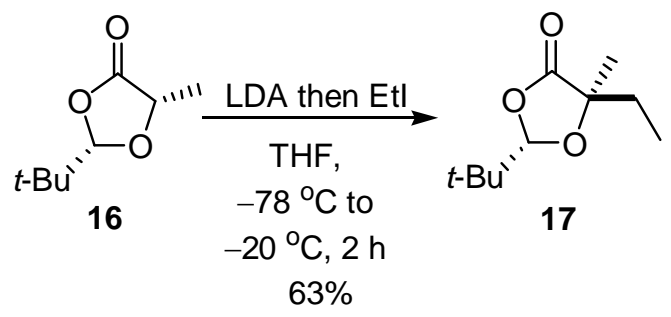

(2S,5R)-2-(tert-Butyl)-5-ethyl-5-methyl-1,3-dioxolan-4-one (17). To a stirred solution of LDA (0.58 $\mathrm{M}$ in THF, $34 \mathrm{~mL}, 19.72 \mathrm{mmol})$ was added $16(1.56 \mathrm{~g}, 9.86 \mathrm{mmol})$ in THF $(5 \mathrm{~mL})$ at $-78{ }^{\circ} \mathrm{C}$. The reaction mixture was stirred at $-78{ }^{\circ} \mathrm{C}$ for $40 \mathrm{~min}$ and then added ethyl iodide $(3.84 \mathrm{~g}, 24.65 \mathrm{mmol})$. The reaction mixture was allowed to warm to $-20{ }^{\circ} \mathrm{C}$ and stirred for another $2 \mathrm{~h}$. The reaction mixture was quenched with saturated aqueous $\mathrm{NH}_{4} \mathrm{Cl}$ and the aqueous phase was extracted with $\mathrm{Et}_{2} \mathrm{O}$. The combined extracts were washed with brine, dried over $\mathrm{MgSO}_{4}$, filtered and concentrated. The residue was purified by silica-gel chromatography (hexane/EtOAc $=30: 1)$ to give $17(1.16 \mathrm{~g}, 63 \%)$ as a colorless liquid. $[\alpha]_{\mathrm{D}}^{25}=+64.8\left(c\right.$ 1.00, $\left.\mathrm{CHCl}_{3}\right)\left[\right.$ lit. $^{1}[\alpha]_{\mathrm{D}}^{25}=+43.8\left(c\right.$ 2.52, $\left.\left.\mathrm{CHCl}_{3}\right)\right]$; IR (neat) $v$ 2977, 2882, 1803, 1486, 1375, 1187, 1081, $979 \mathrm{~cm}^{-1} ;{ }^{1} \mathrm{H}$ NMR (400 MHz, $\left.\mathrm{CDCl}_{3}\right) \delta 5.19$ (s, 1H), 1.90-1.70 (m, $\left.2 \mathrm{H}\right), 1.42$ (s, 3H), $1.00(\mathrm{t}, J=7.6 \mathrm{~Hz}, 3 \mathrm{H}), 0.95(\mathrm{~s}, 9 \mathrm{H}) ;{ }^{13} \mathrm{C} \mathrm{NMR}\left(100 \mathrm{MHz}, \mathrm{CDCl}_{3}\right) \delta 175.9(\mathrm{C}), 108.6(\mathrm{CH})$, $80.3(\mathrm{C}), 34.6(\mathrm{C}), 29.4\left(\mathrm{CH}_{2}\right), 23.3\left(\mathrm{CH}_{3}\right), 22.3\left(\mathrm{CH}_{3}\right), 8.0\left(\mathrm{CH}_{3}\right)$; MS (EI) $\mathrm{m} / z\left(\%\right.$ base peak) $186\left(\mathrm{M}^{+}\right.$, 0.2), 173 (5), 158 (30), 128 (47), 111 (7), 101 (70), 86 (93), 73 (100), 70 (24), 57 (24), 55 (11); HRMS (EI) calcd for $\mathrm{C}_{10} \mathrm{H}_{18} \mathrm{O}_{3}$ 186.1256, Found 186.1255.

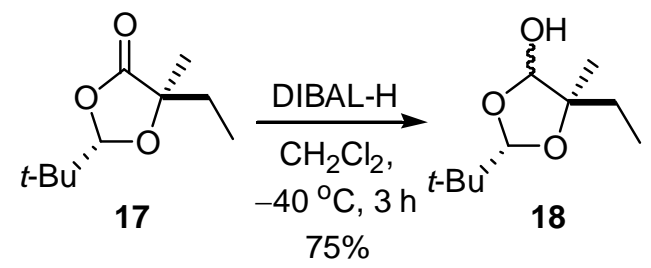

(2S,5R)-2-(tert-Butyl)-5-ethyl-5-methyl-1,3-dioxolan-4-ol (18). To a stirred solution of 17 (435 mg, $2.34 \mathrm{mmol})$ in $\mathrm{CH}_{2} \mathrm{Cl}_{2}(10 \mathrm{~mL})$ was added DIBAL-H (1 M in hexane, $\left.4.25 \mathrm{~mL}, 4.25 \mathrm{mmol}\right)$ at $-40{ }^{\circ} \mathrm{C}$ 
and stirred for $3 \mathrm{~h}$. The reaction mixture was quenched with $10 \% \mathrm{HCl}$. The aqueous phase was extracted with $\mathrm{CH}_{2} \mathrm{Cl}_{2}$. The combined extracts were washed with brine, dried over $\mathrm{MgSO}_{4}$, filtered and concentrated. The residue was purified by silica-gel chromatography (hexane/EtOAc $=3: 1$ ) to give $\mathbf{1 8}$ (330 mg, 75\%) as a colorless liquid. IR (neat) $v 3453,2975,2872,1484,1464,1106,1035,973 \mathrm{~cm}^{-1}$; ${ }^{1} \mathrm{H}$ NMR $\left(400 \mathrm{MHz}, \mathrm{CDCl}_{3}\right) \delta 5.11(\mathrm{~d}, J=3.6 \mathrm{~Hz}, 1 \mathrm{H}), 5.00(\mathrm{~d}, J=7.9 \mathrm{~Hz}, 1 \mathrm{H}), 4.87(\mathrm{~s}, 1 \mathrm{H}), 4.63(\mathrm{~s}$, 1H), 3.55 (brs, 1H), 2.80 (brs, $1 \mathrm{H}), 1.80-1.70(\mathrm{~m}, 1 \mathrm{H}), 1.70-1.55(\mathrm{~m}, 2 \mathrm{H}), 1.53-1.40(\mathrm{~m}, 1 \mathrm{H}), 1.21(\mathrm{~s}$, 3H), 1.19 (s, 3H), 0.96-0.88 (m, 24H); $\left.{ }^{13} \mathrm{C} \mathrm{NMR} \mathrm{(100} \mathrm{MHz,} \mathrm{CDCl}_{3}\right) \delta 108.2(\mathrm{CH}), 107.6(\mathrm{CH}), 100.9$ $(\mathrm{CH}), 98.4(\mathrm{CH}), 84.4(\mathrm{C}), 83.6(\mathrm{C}), 33.62(\mathrm{C}), 33.59(\mathrm{C}), 27.3\left(\mathrm{CH}_{2}\right), 25.8\left(\mathrm{CH}_{2}\right), 24.2\left(\mathrm{CH}_{3}\right), 24.1$ $\left(\mathrm{CH}_{3}\right), 23.0\left(\mathrm{CH}_{3}\right), 18.1\left(\mathrm{CH}_{3}\right), 8.6\left(\mathrm{CH}_{3}\right), 7.6\left(\mathrm{CH}_{3}\right)$; $\mathrm{MS}(\mathrm{EI}) \mathrm{m} / \mathrm{z}\left(\%\right.$ base peak) $188\left(\mathrm{M}^{+}, 0.1\right), 187(1)$, 171 (3), 142 (4), 131 (85), 127 (4), 103 (2), 85 (100), 73 (22); HRMS (EI) calcd for $\mathrm{C}_{10} \mathrm{H}_{20} \mathrm{O}_{3}$ 188.1412, Found 188.1411.

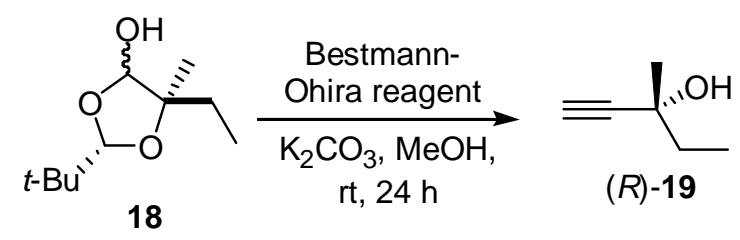

(R)-3-Methylpent-1-yn-3-ol $((\boldsymbol{R})-19)$. A mixture of $18(1.00 \mathrm{~g}, 5.31 \mathrm{mmol})$ and dimethyl (1-diazo-2oxopropyl)phosphonate (Bestmann-Ohira reagent, $3.3 \mathrm{~g}, 17.18 \mathrm{mmol})$ in dry $\mathrm{MeOH}(13 \mathrm{~mL})$ was added $\mathrm{K}_{2} \mathrm{CO}_{3}(2.94 \mathrm{~g}, 21.24 \mathrm{mmol})$ at room temperature and stirred for $24 \mathrm{~h}$. The mixture was then filtered and the filtrate was washed with brine, dried over $\mathrm{MgSO}_{4}$, filtered and concentrated to give a crude $(R)-19$ (320 mg), which was used in the next step without further purification. Analytically pure $(R)$-19 was purified by silica-gel chromatography (pentane/ether $=8: 1$ ) to give $(R)-\mathbf{2 2}$ as a colorless liquid. $[\alpha]^{25}$ $=-4.1\left(c\right.$ 1.00, $\left.\mathrm{CHCl}_{3}\right)\left[\right.$ lit. $^{2}[\alpha]^{23} \mathrm{D}=-1.81$ (neat)]; ${ }^{1} \mathrm{H}$ NMR $\left(400 \mathrm{MHz}, \mathrm{CDCl}_{3}\right) \delta 2.42(\mathrm{~s}, 1 \mathrm{H}), 2.07$ (brs, 1H), 1.77-1.60 (m, 2H), $1.48(\mathrm{~s}, 3 \mathrm{H}), 1.04(\mathrm{t}, J=7.5 \mathrm{~Hz}, 3 \mathrm{H}) ;{ }^{13} \mathrm{C}$ NMR $\left(100 \mathrm{MHz}, \mathrm{CDCl}_{3}\right) \delta 87.5$, $71.2,68.5,36.3,29.1,8.8$. 


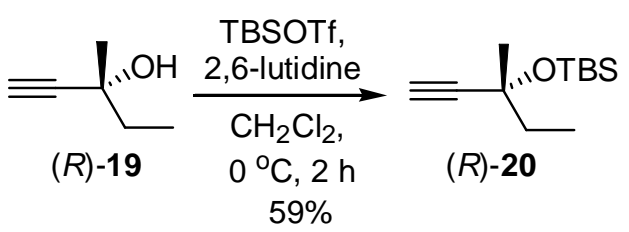

(R)-tert-Butyldimethyl((3-methylpent-1-yn-3-yl)oxy)silane $((\boldsymbol{R})-20)$. To a stirred solution of crude (R)-19 (320 mg, $3.26 \mathrm{mmol})$ in $\mathrm{CH}_{2} \mathrm{Cl}_{2}(8 \mathrm{~mL})$ at $0{ }^{\circ} \mathrm{C}$ was added 2,6 -lutidine $(0.90 \mathrm{~mL}, 7.82 \mathrm{mmol})$ and TBSOTf $(0.90 \mathrm{~mL}, 3.91 \mathrm{mmol})$. The reaction mixture was stirred at $0{ }^{\circ} \mathrm{C}$ for $2 \mathrm{~h}$ and then quenched with dry methanol $(5 \mathrm{~mL})$ and stirred for another $30 \mathrm{~min}$. The solvent was removed in vacuo to give a residue. The crude product was purified by silica-gel column chromatography (hexane) to give $(R)-\mathbf{2 0}(664 \mathrm{mg}$, $59 \%$ over 2 steps $)$ as a colorless liquid. $[\alpha]^{25}{ }_{\mathrm{D}}=+3.7\left(c 1.00, \mathrm{CHCl}_{3}\right) ;{ }^{1} \mathrm{H} \mathrm{NMR}\left(400 \mathrm{MHz}, \mathrm{CDCl}_{3}\right) \delta$ 2.39 (s, 1H), 1.68-1.60 (m, 2H), 1.43 (s, $3 \mathrm{H}), 0.99$ (t, J = $7.3 \mathrm{~Hz}, 3 \mathrm{H}), 0.87(\mathrm{~s}, 9 \mathrm{H}), 0.17(\mathrm{~s}, 6 \mathrm{H}),{ }^{13} \mathrm{C}$ NMR (100 MHz, $\left.\mathrm{CDCl}_{3}\right) \delta 88.2,71.7,69.5,38.0,30.5,25.7,18.1,8.8,-2.9,-3.2$.

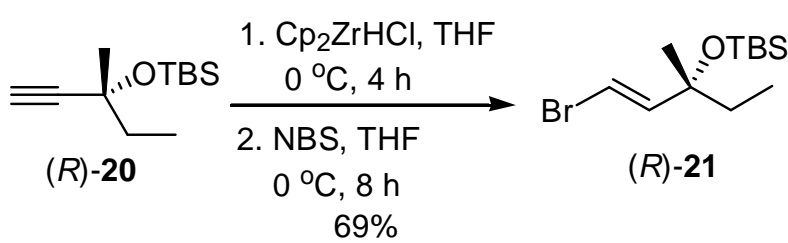

$(\boldsymbol{R})-(\boldsymbol{E})-((1-B r o m o-3-m e t h y l p e n t-1-e n-3-y l) o x y) t e r t-b u t y l d i m e t h y l s i l a n e \quad((R)-21) . \quad$ To $\quad$ a $\quad$ stirred solution of zirconocene chloride hydride, which was prepared from zirconocene dichloride (790 mg, $2.70 \mathrm{mmol})$ in dry THF $(15 \mathrm{~mL})$ and DIBAL-H $(2.5 \mathrm{~mL}, 1.05 \mathrm{M}$ in hexane, $2.63 \mathrm{mmol})$ at $0{ }^{\circ} \mathrm{C}$ for $1 \mathrm{~h}$, was added $(R)-\mathbf{2 0}(570 \mathrm{mg}, 2.68 \mathrm{mmol})$ and stirred at $0{ }^{\circ} \mathrm{C}$ for $4 \mathrm{~h}$. NBS $(650 \mathrm{mg}, 3.65 \mathrm{mmol})$ was added to the reaction mixture at $0{ }^{\circ} \mathrm{C}$ and stirred for another $8 \mathrm{~h}$. The reaction mixture was added hexanes (30 $\mathrm{mL}$ ) and then filtered. The filtrate was wished with saturated aqueous $\mathrm{NH}_{4} \mathrm{Cl}$, brine, dried over $\mathrm{MgSO}_{4}$, 
filtered and concentrated. The residue was purified by chromatography on alumina (hexane) to give $(R)$ $21(547 \mathrm{mg}, 69 \%)$ as a colorless liquid. $[\alpha]^{25}{ }_{\mathrm{D}}=+13.8\left(c 1.00, \mathrm{CHCl}_{3}\right) ;{ }^{1} \mathrm{H} \mathrm{NMR}\left(500 \mathrm{MHz}, \mathrm{CD}_{2} \mathrm{Cl}_{2}\right) \delta$ $6.23(\mathrm{~d}, J=13.5 \mathrm{~Hz}, 1 \mathrm{H}), 6.19(\mathrm{~d}, J=13.5 \mathrm{~Hz}, 1 \mathrm{H}), 1.60-1.45(\mathrm{~m}, 2 \mathrm{H}), 1.30(\mathrm{~s}, 3 \mathrm{H}), 0.89(\mathrm{~s}, 9 \mathrm{H}), 0.87$ $(\mathrm{t}, J=7.2 \mathrm{~Hz}, 3 \mathrm{H}), 0.10(\mathrm{~s}, 3 \mathrm{H}), 0.09(\mathrm{~s}, 3 \mathrm{H}) ;{ }^{13} \mathrm{C} \mathrm{NMR}\left(125 \mathrm{MHz}, \mathrm{CD}_{2} \mathrm{Cl}_{2}\right) \delta 145.3,105.2,77.7,36.6$, $27.7,26.3,18.8,8.7,-1.8,-1.9$.
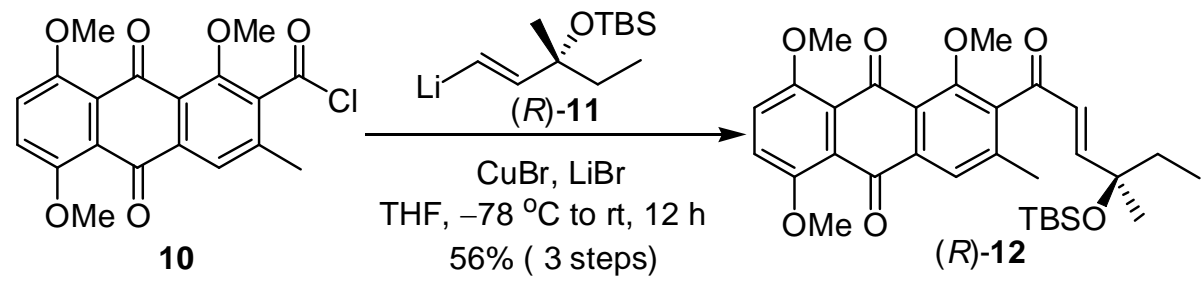

$(R)-12$

\section{(R)-2-[3-(tert-Butyldimethylsilyloxy)-3-methyl-1-pentenyl]-1,5,8-trimethoxy-3-}

methylanthraquinone $((\boldsymbol{R})-\mathbf{1 2})$. To a stirred solution of $(R)-\mathbf{1 1}(0.85 \mathrm{mmol})$ in $\mathrm{THF}$ at $-78{ }^{\circ} \mathrm{C}$ which was prepared from $(\boldsymbol{R})-\mathbf{2 1}(250 \mathrm{mg}, 0.85 \mathrm{mmol})$ in THF $(10 \mathrm{~mL})$ and tert-BuLi (1.94 $\mathrm{M}$ in pentane, 0.85 $\mathrm{mL}, 1.65 \mathrm{mmol})$ at $-78{ }^{\circ} \mathrm{C}$ for $1 \mathrm{~h}$, was added a solution of $\mathrm{CuBr}(122 \mathrm{mg}, 0.85 \mathrm{mmol})$ and $\mathrm{LiBr}(150$ $\mathrm{mg}, 1.72 \mathrm{mmol})$ in THF (5 mL) and this solution was then added to a solution of crude acid chloride $\mathbf{1 0}$ (100 mg, $0.27 \mathrm{mmol})$ in THF $(5 \mathrm{~mL})$. The reaction mixture was then allowed to warm to room temperature and stirred for $12 \mathrm{~h}$. The mixture was quenched with saturated aqueous $\mathrm{NH}_{4} \mathrm{Cl}$. The aqueous solution was extracted with $\mathrm{CH}_{2} \mathrm{Cl}_{2}$, and the combined extracts were washed with brine, dried over $\mathrm{MgSO}_{4}$, filtered and concentrated. The residue was purified by silica-gel chromatography (hexane/EtOAc $=2: 3)$ to afford $(R)-\mathbf{1 2}(93 \mathrm{mg}, 56 \%$, over 3 steps) as a yellow solid: ee $=91 \%$ (HPLC column CHIRALPAK IC; injection amount $20 \mu \mathrm{L}$; mobile phase hexane/EtOAc (65/35 v/v); flow rate 1 $\left.\mathrm{mL} / \mathrm{min}, t_{\text {major }}=25.25 \mathrm{~min}, t_{\text {minor }}=27.85 \mathrm{~min}\right) ;[\alpha]_{\mathrm{D}}^{25}=+10.0\left(c 1.00, \mathrm{CHCl}_{3}\right) ; \mathrm{Mp} 175-176{ }^{\circ} \mathrm{C} ;{ }^{1} \mathrm{H}$ $\operatorname{NMR}\left(500 \mathrm{MHz}, \mathrm{CDCl}_{3}\right) \delta 7.76(\mathrm{~s}, 1 \mathrm{H}), 7.31(\mathrm{~d}, J=9.5 \mathrm{~Hz}, 1 \mathrm{H}), 7.26(\mathrm{~d}, J=9.5 \mathrm{~Hz}, 1 \mathrm{H}), 6.49(\mathrm{~d}, J=$ $15.8 \mathrm{~Hz}, 1 \mathrm{H}), 6.42(\mathrm{~d}, J=15.8 \mathrm{~Hz}, 1 \mathrm{H}), 3.98(\mathrm{~s}, 3 \mathrm{H}), 3.96(\mathrm{~s}, 3 \mathrm{H}), 3.87(\mathrm{~s}, 3 \mathrm{H}), 2.28(\mathrm{~s}, 3 \mathrm{H}), 1.60-1.50$ 
$(\mathrm{m}, 2 \mathrm{H}), 1.31(\mathrm{~s}, 3 \mathrm{H}), 0.88(\mathrm{~s}, 9 \mathrm{H}), 0.81(\mathrm{t}, J=7.3 \mathrm{~Hz}, 3 \mathrm{H}), 0.07(\mathrm{~s}, 3 \mathrm{H}), 0.05(\mathrm{~s}, 3 \mathrm{H}) ;{ }^{13} \mathrm{C} \mathrm{NMR}(125$ $\left.\mathrm{MHz}, \mathrm{CDCl}_{3}\right) \delta 196.8,183.2,182.7,158.5,156.3,153.4,153.0,141.6,140.4,136.3,128.7,125.4,125.3$, $123.9,122.5,120.3,118.8,75.7,63.6,57.2,56.9,35.8,27.4,25.9,19.5,18.4,8.3,-2.1,-2.2$.<smiles>CCO[C@@](C)(/C=C/C(=O)c1c(C)cc2c(c1OC)C(=O)c1c(OC)ccc(OC)c1C2=O)CC</smiles>

$(R)-12$

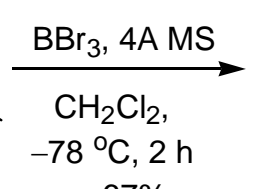

$67 \%$

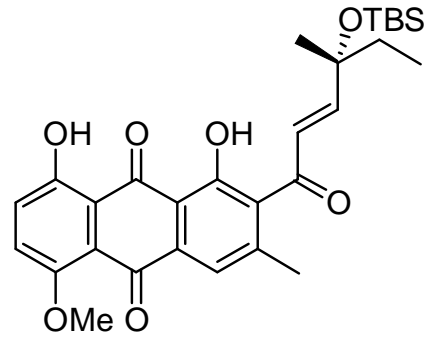

$(R)-4$

\section{(R)-(E)-2-(4-((tert-butyldimethylsilyl)oxy)-4-methylhex-2-enoyl)-1,8-dihydroxy-5-methoxy-3-}

methylanthraquinone $((\boldsymbol{R})-4)$. To a stirred mixture of $(R)-12(60 \mathrm{mg}, 0.11 \mathrm{mmol})$ and dry molecular sieve $4 \AA(0.80 \mathrm{~g})$ in $\mathrm{CH}_{2} \mathrm{Cl}_{2}(10 \mathrm{~mL})$ at $-78{ }^{\circ} \mathrm{C}$ was added $\mathrm{BBr}_{3}\left(1 \mathrm{M}\right.$ in $\left.\mathrm{CH}_{2} \mathrm{Cl}_{2}, 0.33 \mathrm{~mL}, 0.33 \mathrm{mmol}\right)$. After stirred at $-78{ }^{\circ} \mathrm{C}$ for $2 \mathrm{~h}$, the mixture was quenched with water. The aqueous phase was extracted with $\mathrm{CH}_{2} \mathrm{Cl}_{2}$. The combined extracts were washed with brine, dried over $\mathrm{MgSO}_{4}$, filtered and concentrated. The crude product was purified by gradient silica-gel chromatography (hexane/ $\mathrm{CH}_{2} \mathrm{Cl}_{2}=$ $1: 3$ to $0: 1)$ to give $(R)-4(38 \mathrm{mg}, 67 \%)$ as a red solid. Analytically pure $(R)-\mathbf{4}$ was obtained by crystallization from $\mathrm{CH}_{2} \mathrm{Cl}_{2}$-hexane: $[\alpha]^{25} \mathrm{D}=-10.9$ (c 1.00, $\left.\mathrm{CHCl}_{3}\right) ; \mathrm{Mp} 158-159{ }^{\circ} \mathrm{C} ;{ }^{1} \mathrm{H} \mathrm{NMR}(500$ $\left.\mathrm{MHz}, \mathrm{CDCl}_{3}\right) \delta 13.19(\mathrm{~s}, 1 \mathrm{H}), 13.07(\mathrm{~s}, 1 \mathrm{H}), 7.67(\mathrm{~s}, 1), 7.42(\mathrm{~d}, J=9.5 \mathrm{~Hz}, 1 \mathrm{H}), 7.35(\mathrm{~d}, J=9.5 \mathrm{~Hz}$, 1H), 6.54-6.52 (m, 2H), $4.04(\mathrm{~s}, 3 \mathrm{H}), 2.33(\mathrm{~s}, 3 \mathrm{H}), 1.62-1.50(\mathrm{~m}, 2 \mathrm{H}), 1.34(\mathrm{~s}, 3 \mathrm{H}), 0.88(\mathrm{~s}, 9 \mathrm{H}), 0.83(\mathrm{t}$, $J=7.3 \mathrm{~Hz}, 1 \mathrm{H}), 0.07(\mathrm{~s}, 3 \mathrm{H}), 0.04(\mathrm{~s}, 3 \mathrm{H}) ;{ }^{13} \mathrm{C} \mathrm{NMR}\left(125 \mathrm{MHz}, \mathrm{CDCl}_{3}\right) \delta 196.1(\mathrm{C}), 187.8(\mathrm{C}), 187.3$ (C), $159.7(\mathrm{C}), 158.2(\mathrm{CH}), 157.9(\mathrm{C}), 155.0(\mathrm{C}), 144.3(\mathrm{C}), 135.5(\mathrm{C}), 132.1(\mathrm{C}), 128.5(\mathrm{CH}), 127.6$ $(\mathrm{CH}), 123.8(\mathrm{CH}), 120.2(\mathrm{CH}), 118.1(\mathrm{C}), 115.5(\mathrm{C}), 115.1(\mathrm{C}), 75.7(\mathrm{C}), 57.0(\mathrm{C}), 35.9\left(\mathrm{CH}_{2}\right), 27.3$ $\left(\mathrm{CH}_{3}\right), 25.9\left(\mathrm{CH}_{3}\right), 19.9(\mathrm{C}), 18.3\left(\mathrm{CH}_{3}\right), 8.3\left(\mathrm{CH}_{3}\right),-2.1\left(\mathrm{CH}_{3}\right),-2.2\left(\mathrm{CH}_{3}\right)$. 


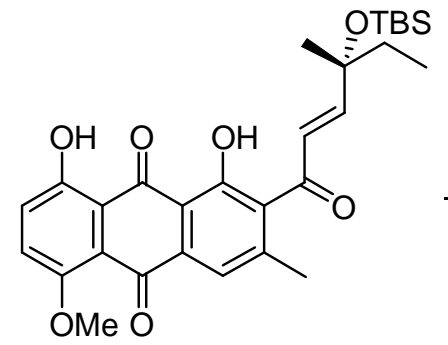

$(R)-4$
1. $\mathrm{I}_{2}, \mathrm{DMSO}, 160^{\circ} \mathrm{C}, 24 \mathrm{~h}$

2. $\mathrm{BBr}_{3}, \mathrm{CH}_{2} \mathrm{Cl}_{2},-78^{\circ} \mathrm{C}$

$$
\text { to }-50{ }^{\circ} \mathrm{C}, 3 \mathrm{~h}
$$

3. $\mathrm{Na}_{2} \mathrm{~S}_{2} \mathrm{O}_{4}, \mathrm{TBAB}$

$\mathrm{CH}_{2} \mathrm{Cl}_{2} / \mathrm{H} 2 \mathrm{O}(2 / 1)$, rt, $1 \mathrm{~h}$ $18 \%$ (3 steps)

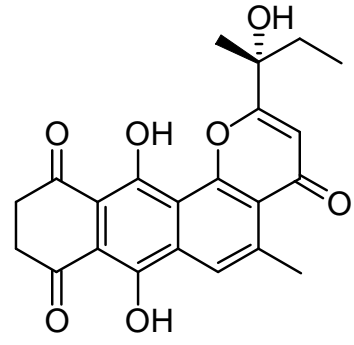

$(R)-(-)$-parimycin $((R)-(-)-1)$

$(\boldsymbol{R})$-Parimycin $((\boldsymbol{R})-\mathbf{1})$. To a stirred solution of $(R)-\mathbf{4}(20 \mathrm{mg}, 0.038 \mathrm{mmol})$ in dry DMSO $(2.5 \mathrm{~mL})$ was added $\mathrm{I}_{2}(5 \mathrm{mg}, 0.019 \mathrm{mmol})$ at room temperature. The reaction mixture was heated to $160{ }^{\circ} \mathrm{C}$ for $24 \mathrm{~h}$. After cooled to room temperature, the reaction mixture was diluted with $\mathrm{CH}_{2} \mathrm{Cl}_{2}$. The reaction mixture was washed with water and brine, dried over $\mathrm{MgSO}_{4}$, filtered and concentrated to give $(R)-\mathbf{1 4}(6.6 \mathrm{mg})$ as a red solid, which was used in the next step without further purification. To a stirred solution of crdue $(R)-14(6.6 \mathrm{mg}, 0.016 \mathrm{mmol})$ in $\mathrm{CH}_{2} \mathrm{Cl}_{2}(3 \mathrm{~mL})$ at $-78{ }^{\circ} \mathrm{C}$ was added $\mathrm{BBr}_{3}\left(1 \mathrm{M}\right.$ in $\mathrm{CH}_{2} \mathrm{Cl}_{2}, 0.14 \mathrm{~mL}$, $0.14 \mathrm{mmol}$ ). The mixture was allowed to warm to $-50{ }^{\circ} \mathrm{C}$ and stirred at this temperature for $3 \mathrm{~h}$. The mixture was quenched with water and extracted with $\mathrm{CH}_{2} \mathrm{Cl}_{2}$. The combined extracts were washed with brine, dried over $\mathrm{MgSO}_{4}$, filtered and concentrated to give $(R)-3(3.7 \mathrm{mg})$ as a red solid, which was used in the next step without further purification. To a stirred mixture of sodium dithionite $(20 \mathrm{mg}, 0.115$ $\mathrm{mmol})$, and TBAB $(2 \mathrm{mg}, 0.006 \mathrm{mmol})$ in $\mathrm{H}_{2} \mathrm{O}(1 \mathrm{~mL})$ was added a solution of crude $(R)-3(3.7 \mathrm{mg}$, $0.009 \mathrm{mmol})$ in $\mathrm{CH}_{2} \mathrm{Cl}_{2}(2 \mathrm{~mL})$ at room temperature. The reaction mixture was stirred at room temperature for $1 \mathrm{~h}$ and then quenched with water. The aqueous phase was extracted with $\mathrm{CH}_{2} \mathrm{Cl}_{2}$. The combined extracts were washed with brine, dried over $\mathrm{MgSO}_{4}$, filtered and concentrated. The crude product was purified by silica-gel chromatography $\left(\mathrm{CH}_{2} \mathrm{Cl}_{2} / \mathrm{EtOAc}=9: 1\right)$ to give $(R)-\mathbf{1}(2.7 \mathrm{mg}, 18 \%$ over 3 steps) as a yellow solid: ee $=95 \%$ (HPLC column CHIRALPAK IC; injection amount $20 \mu \mathrm{L}$; mobile phase hexane/THF $(82 / 18 \mathrm{v} / \mathrm{v})$; flow rate $\left.1 \mathrm{~mL} / \mathrm{min}, t_{\text {major }}=73.17 \mathrm{~min}, t_{\text {minor }}=66.27 \mathrm{~min}\right) ;[\alpha]_{\mathrm{D}}^{25}$ $=-24.0\left(c\right.$ 0.03, MeOH); $\mathrm{Mp}>115{ }^{\circ} \mathrm{C}($ dec $) ;{ }^{1} \mathrm{H}$ NMR $\left(400 \mathrm{MHz}, \mathrm{CDCl}_{3}\right) \delta 14.72(\mathrm{~s}, 1 \mathrm{H}), 13.38(\mathrm{~s}$, 
1H), $8.16(\mathrm{~s}, 1 \mathrm{H}), 6.54(\mathrm{~s}, 1 \mathrm{H}), 3.13(\mathrm{~s}, 4 \mathrm{H}), 3.03(\mathrm{~s}, 3 \mathrm{H}), 2.10-2.00(\mathrm{~m}, 1 \mathrm{H}), 2.00-1.90(\mathrm{~m}, 1 \mathrm{H}), 1.64$ (s, $3 \mathrm{H}), 0.95(\mathrm{t}, J=7.6 \mathrm{~Hz}, 3 \mathrm{H})$.

\section{References in Supporting Information}

1. Seebach, D.; Naff, R.; Calderari, G. Tetrahedron 1984, 40, 1313-1324.

2. Faulkner, D. J.; Petersen, M. R. J. Am. Chem. Soc. 1973, 95, 553-563. 


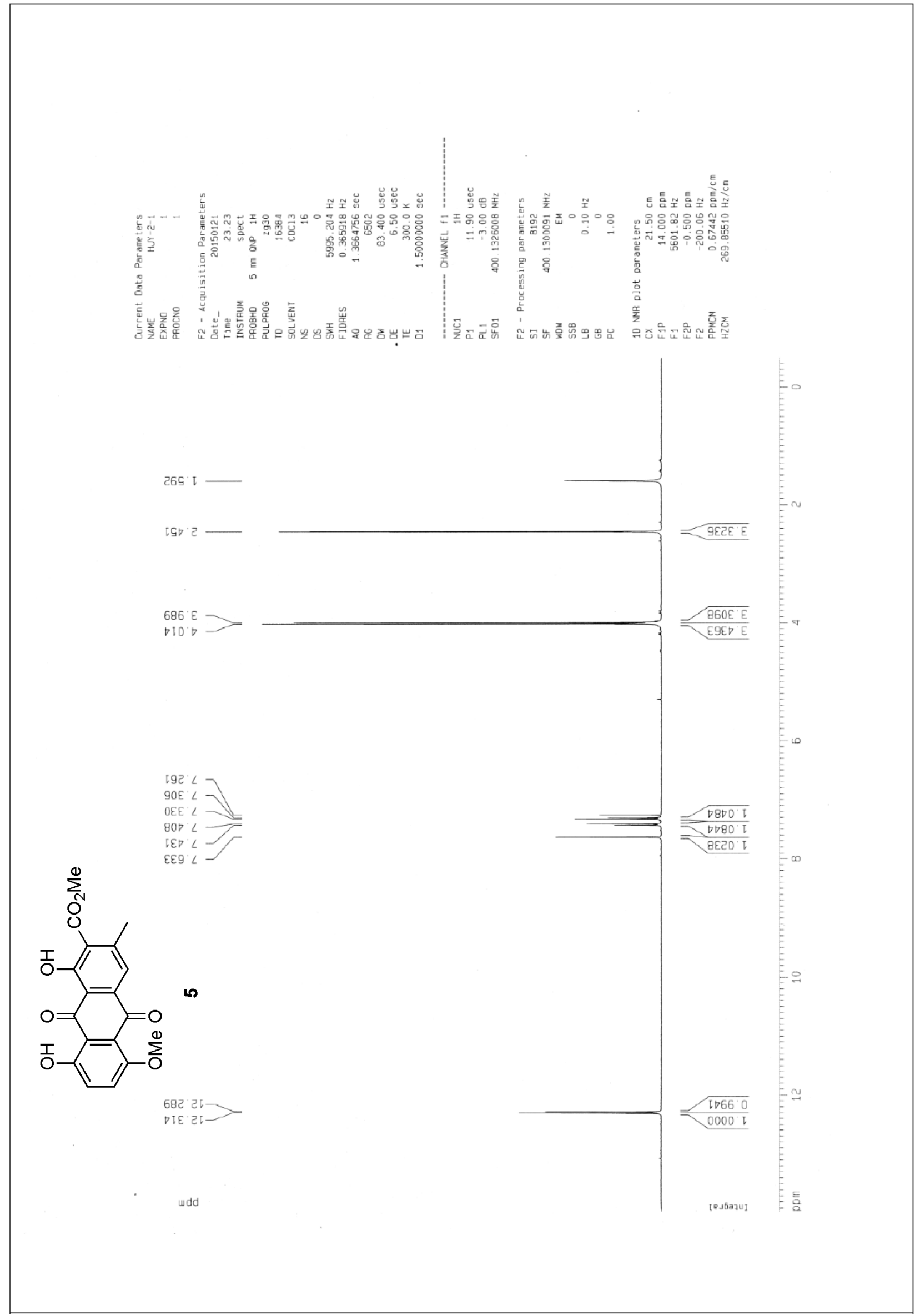




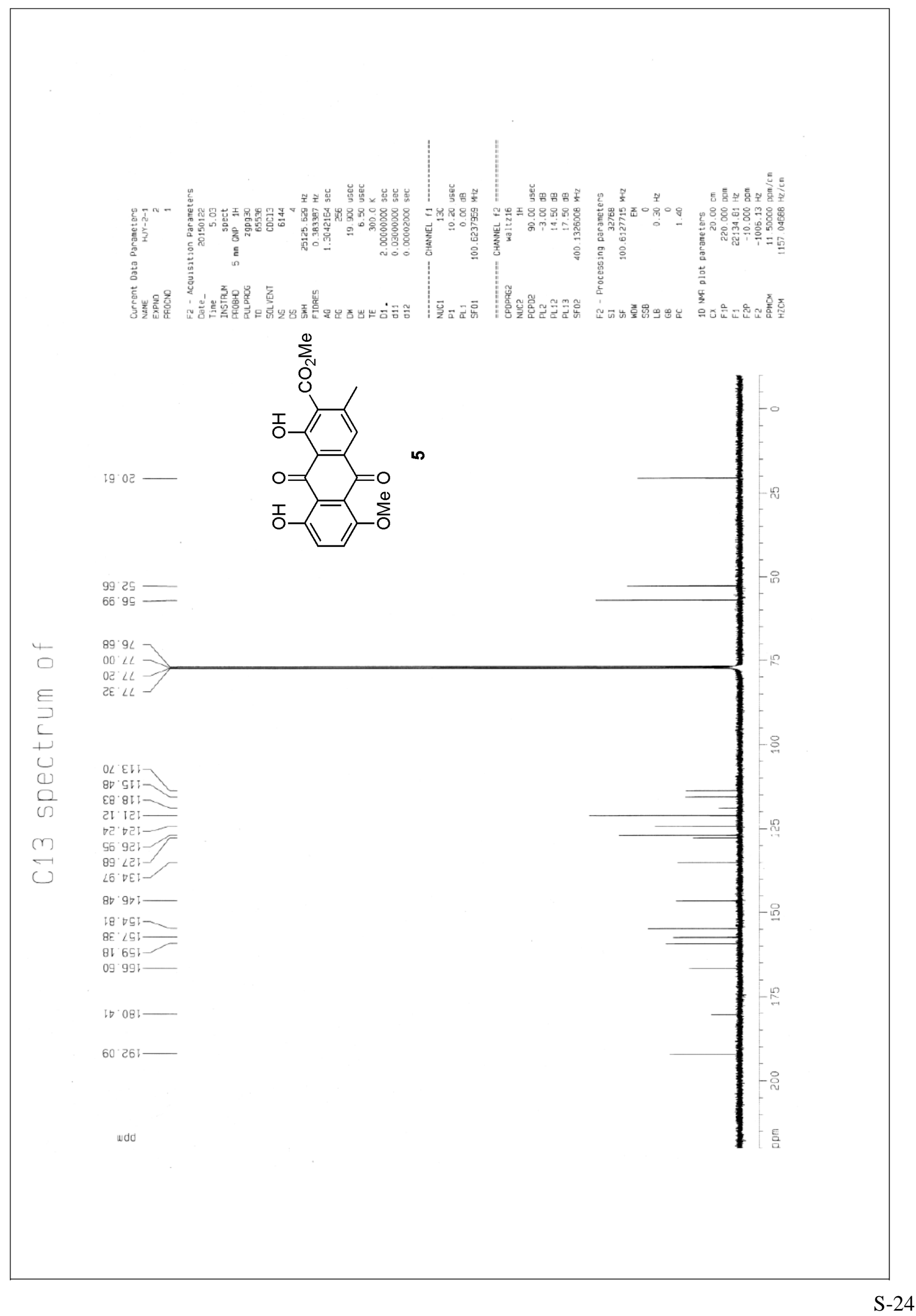




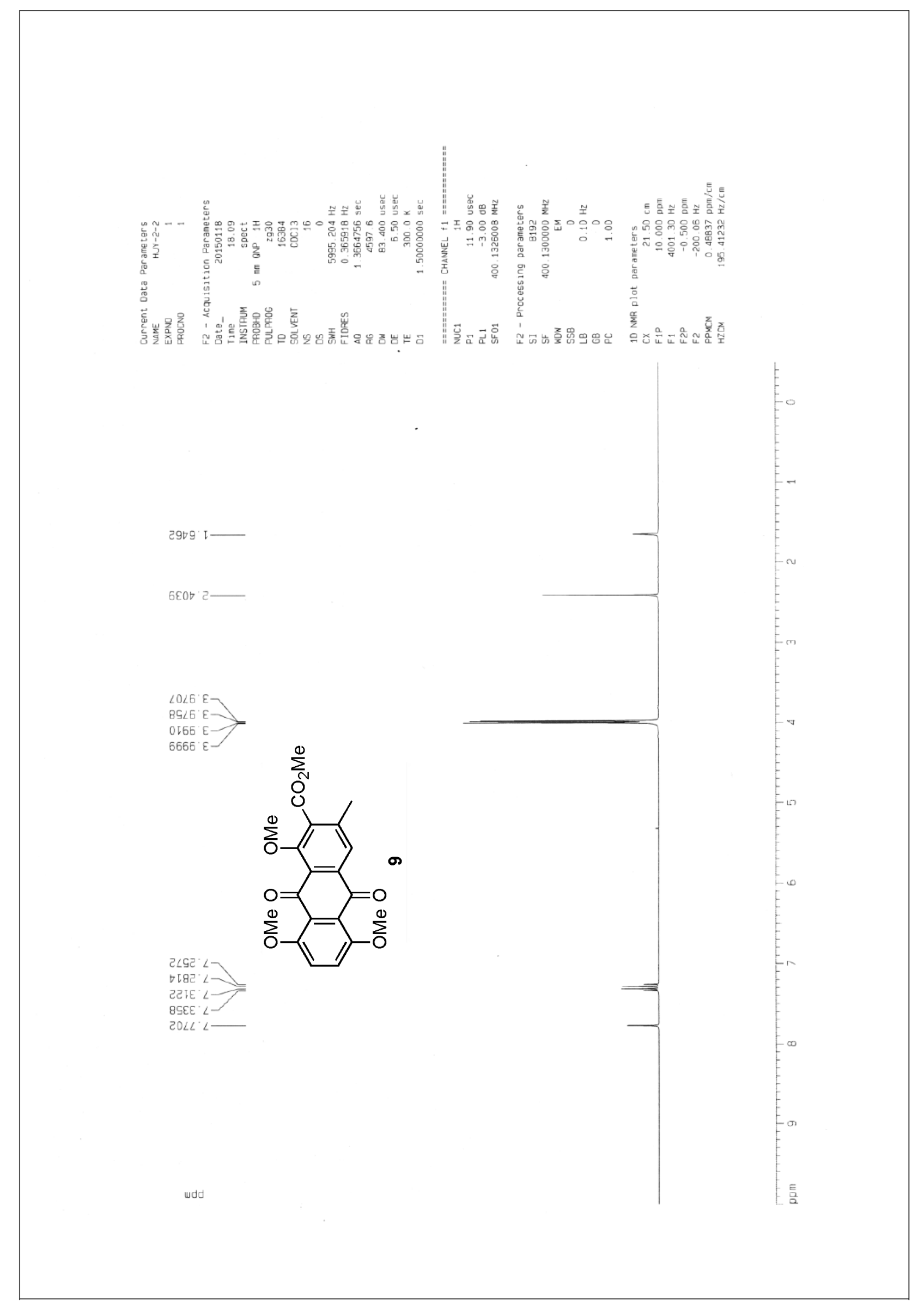




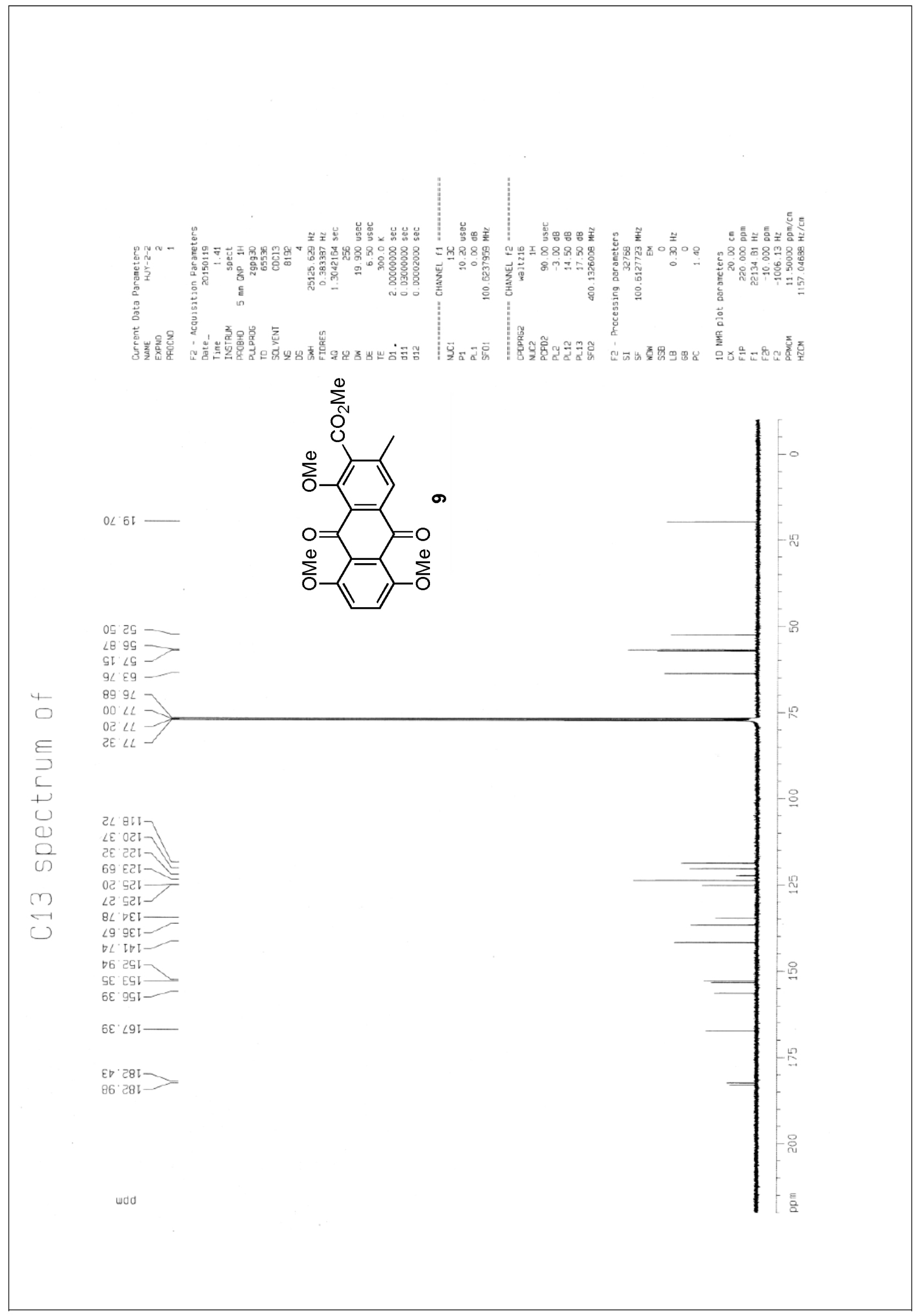



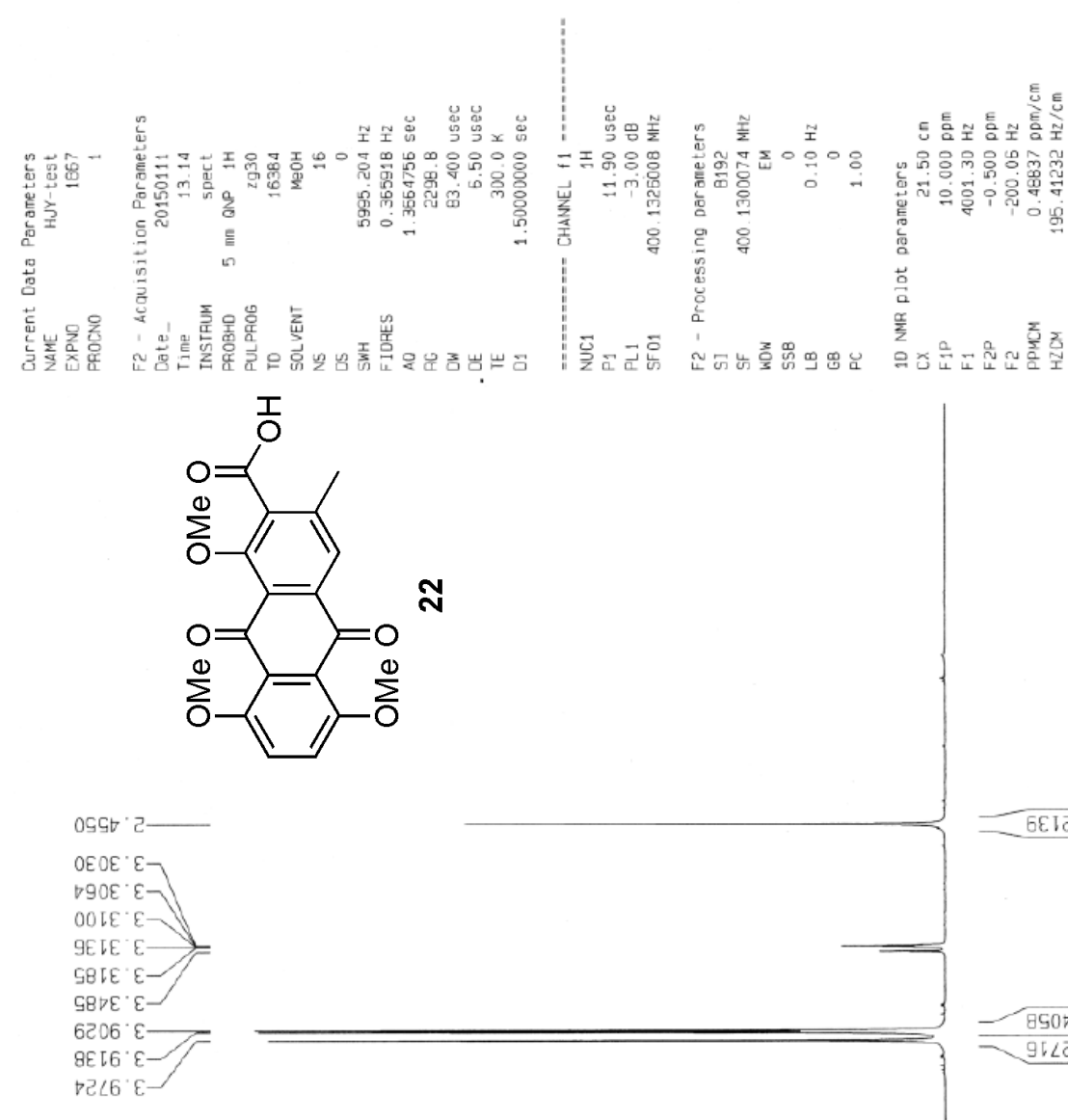

$9898^{\circ}$

$888 \varepsilon$

Gटto.

rQE

Iटक口 1

$62 \angle 9<1$

wdd

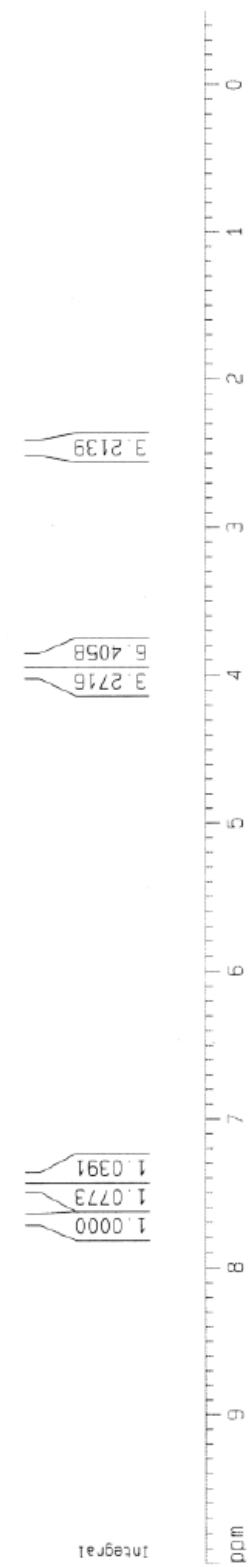




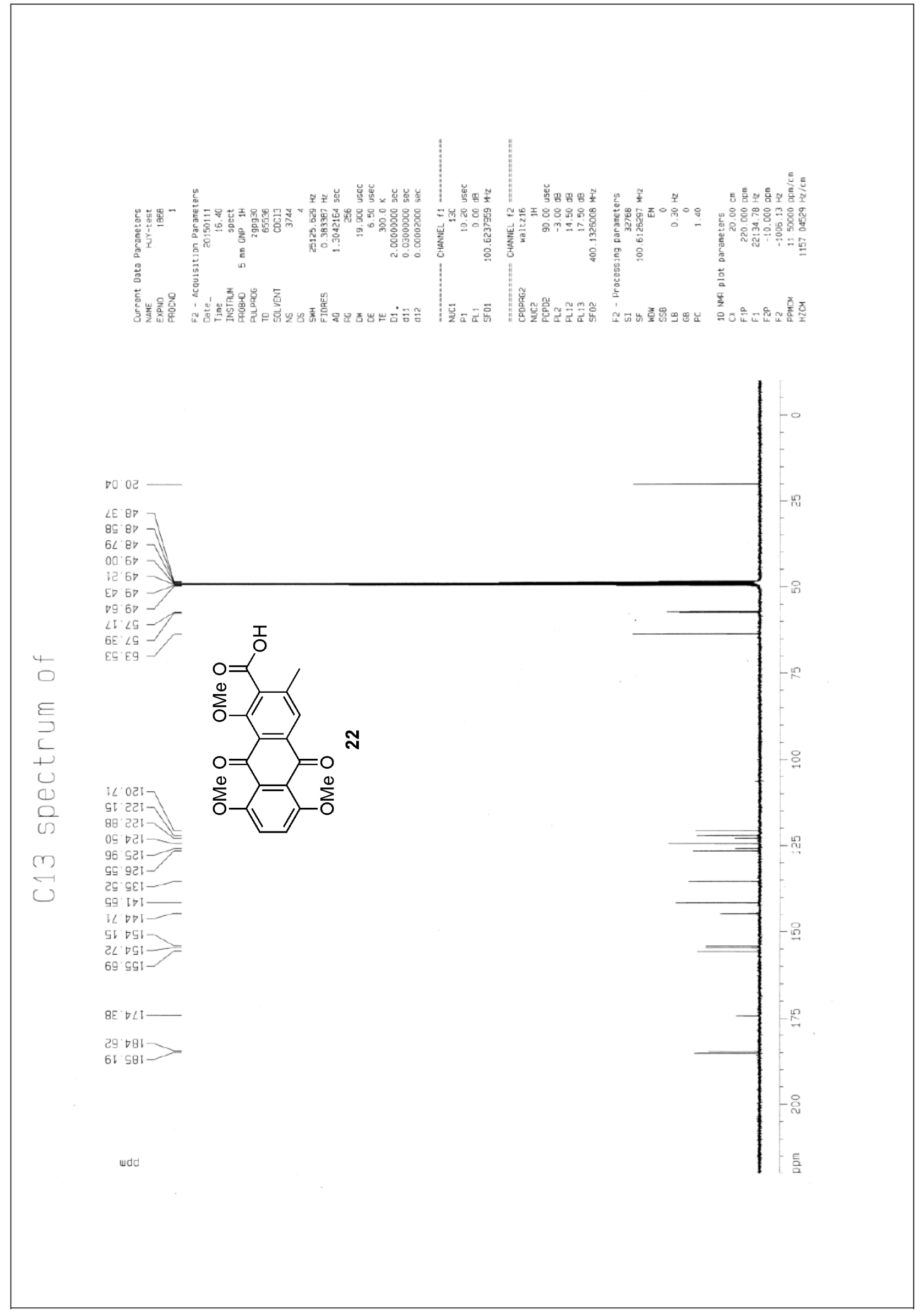




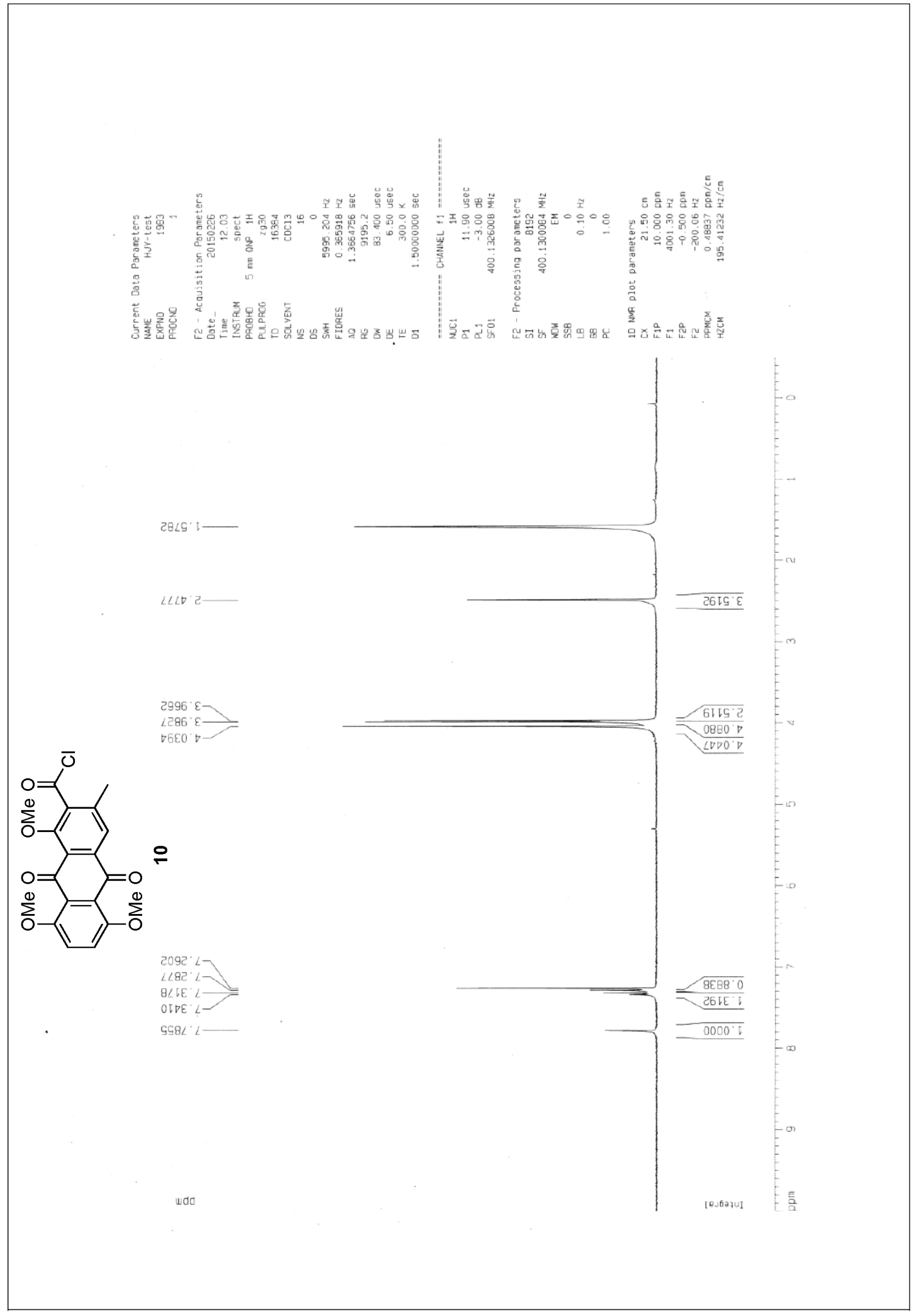




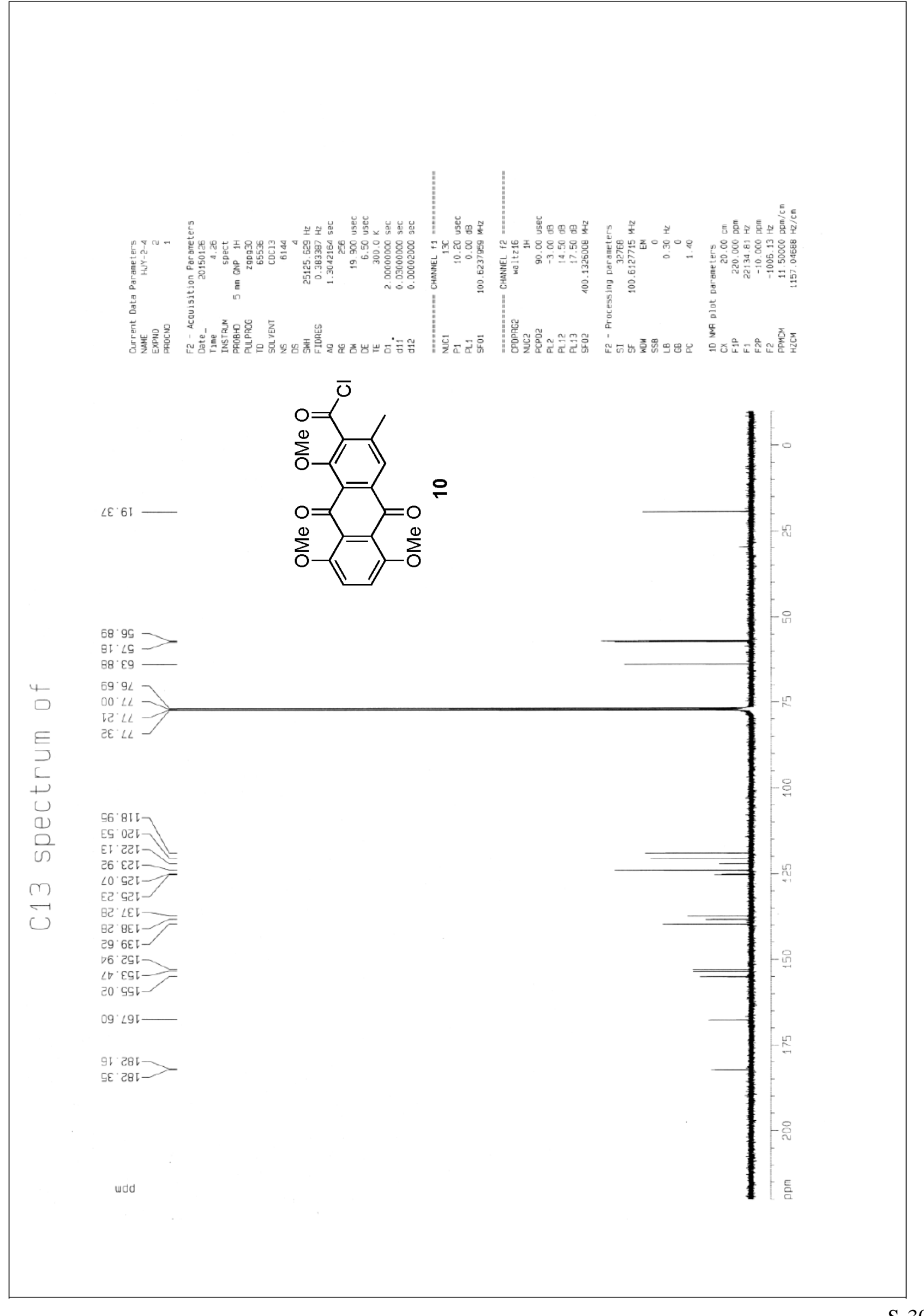




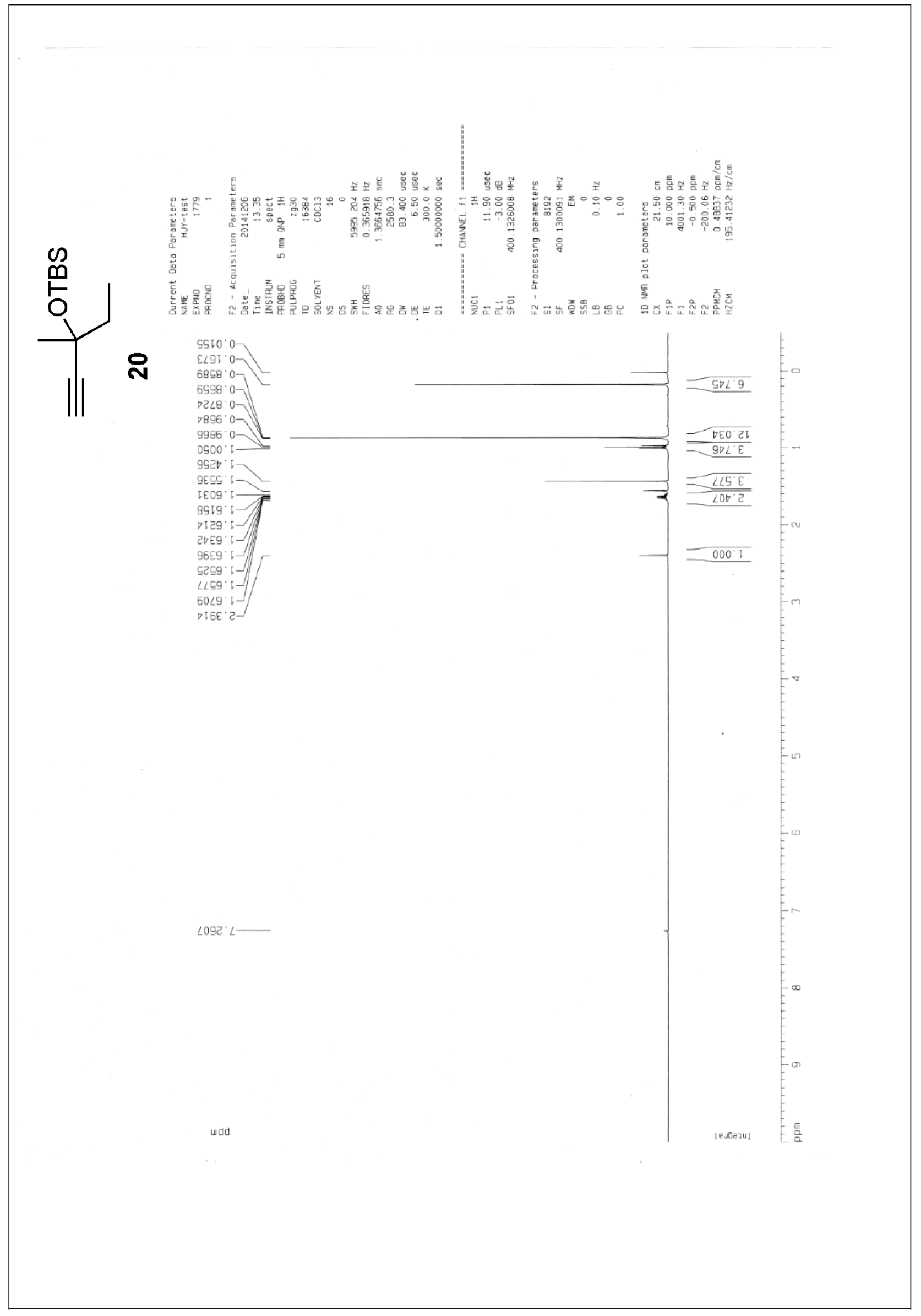




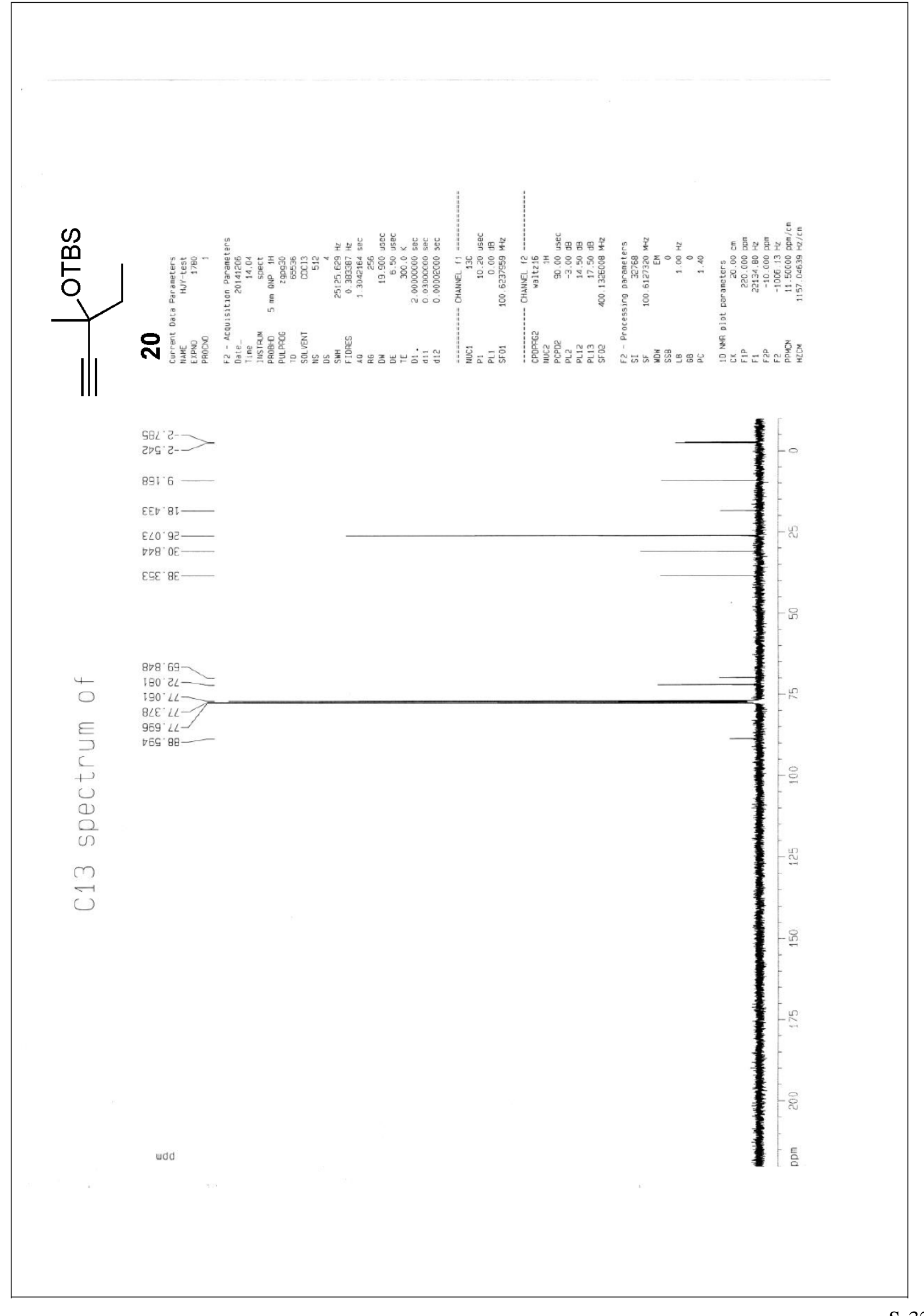



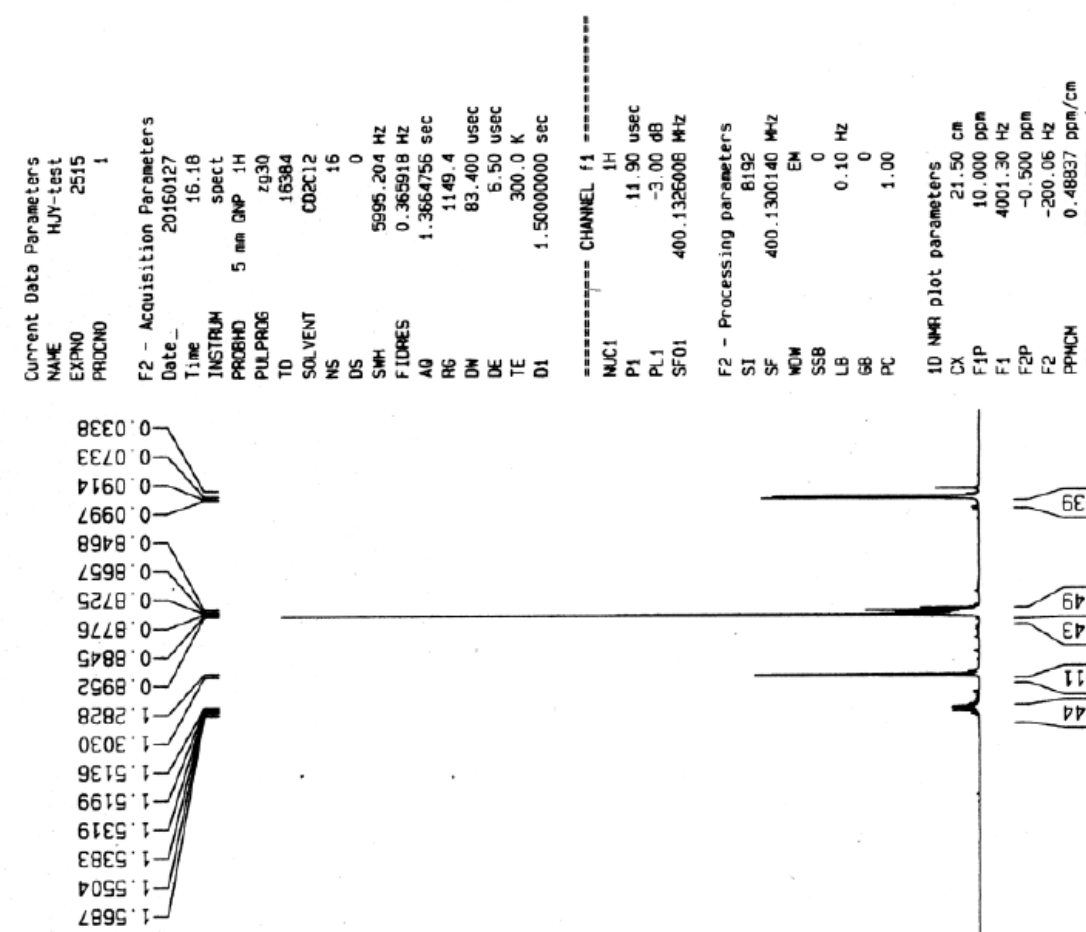

$\angle O C E^{\circ} S-$

$00<19$

$\checkmark E 0 C^{\circ} \cdot 9$

ह9.ट 9

$66 \nabla 29$

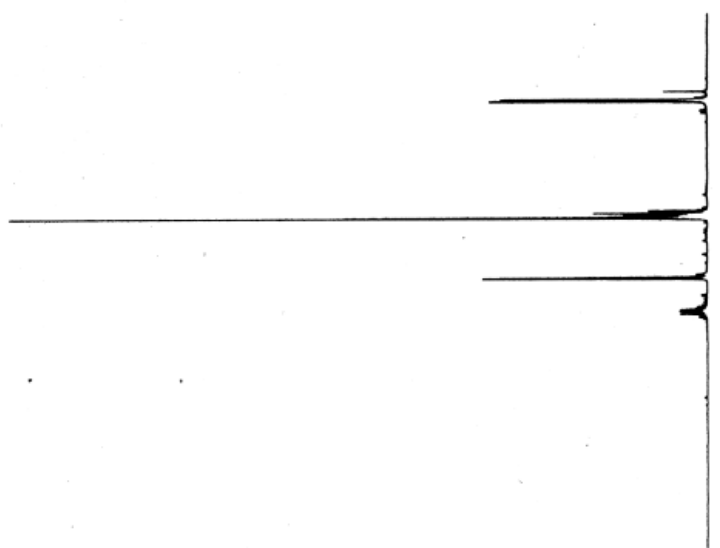

(1) $\widetilde{\text { EEE } 0}$ $\sqrt{6 t \angle 8 t}$ $\begin{array}{r}\text { ERO } 0 \\ \hline 1500\end{array}$

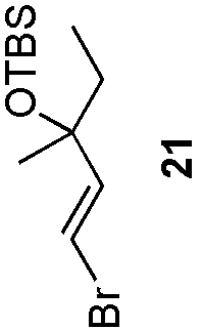

wdd 


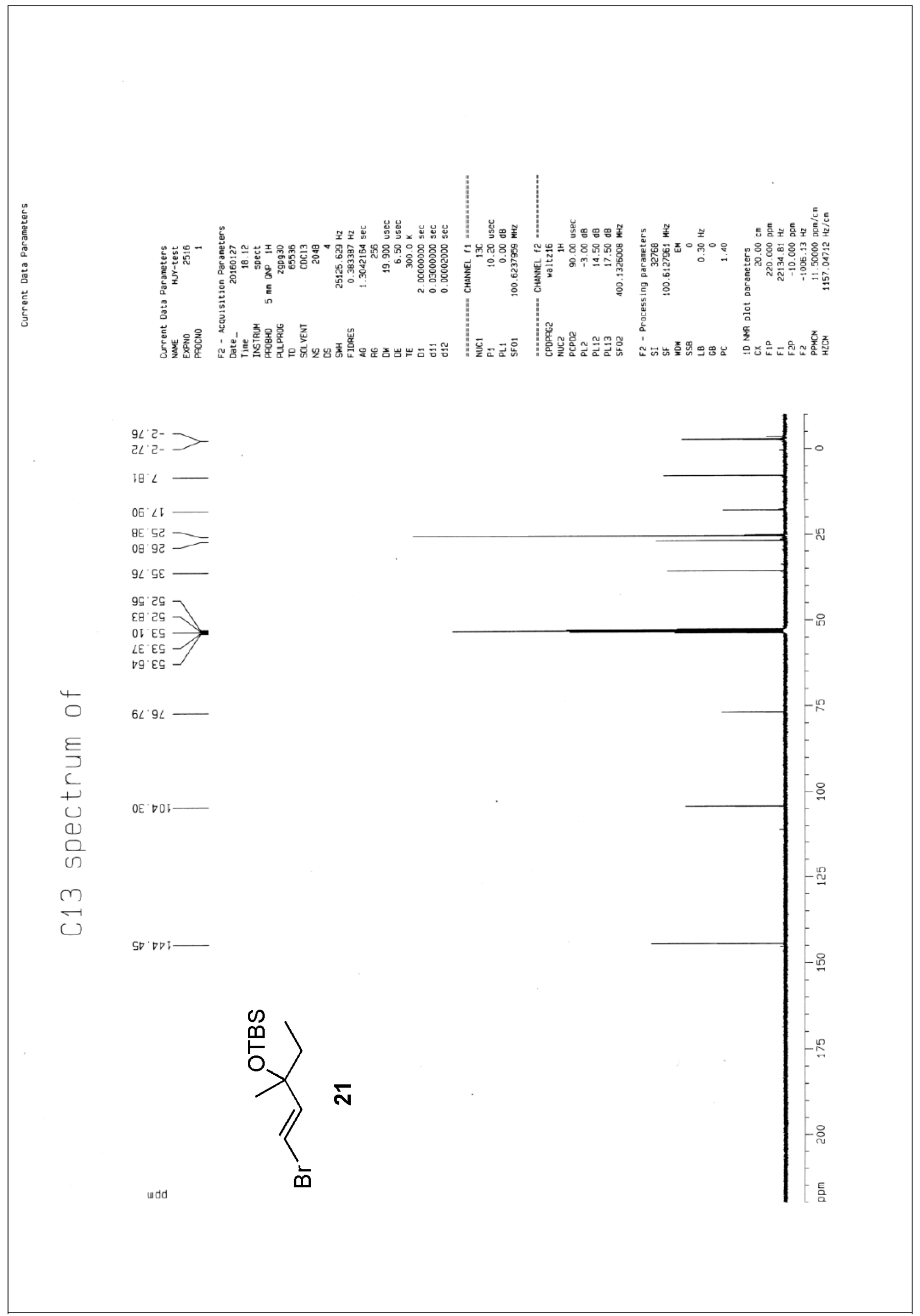



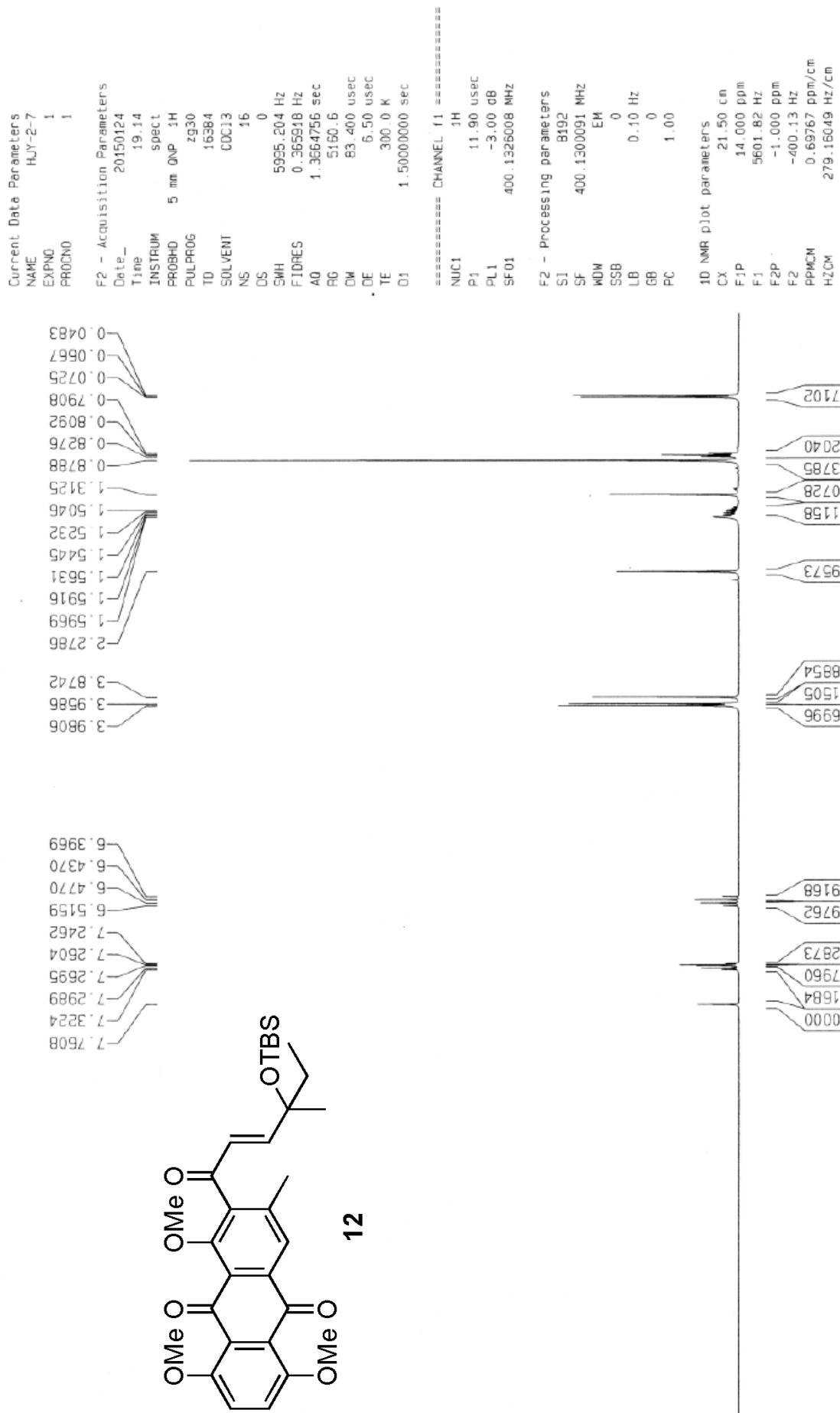

udd

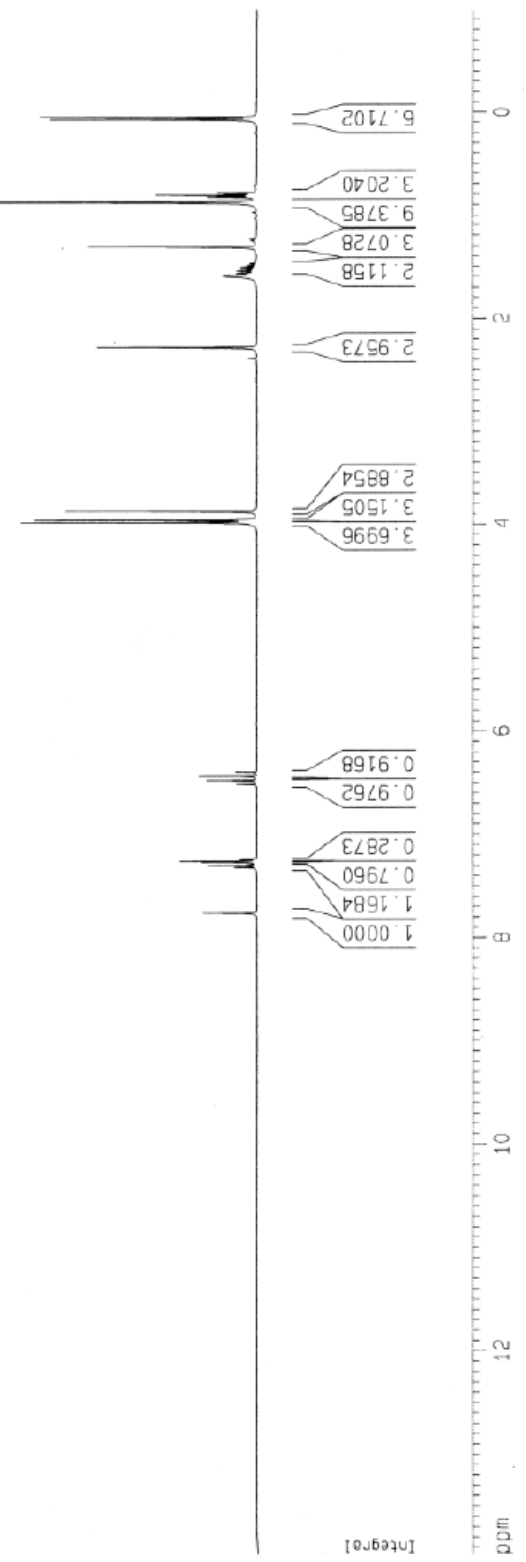




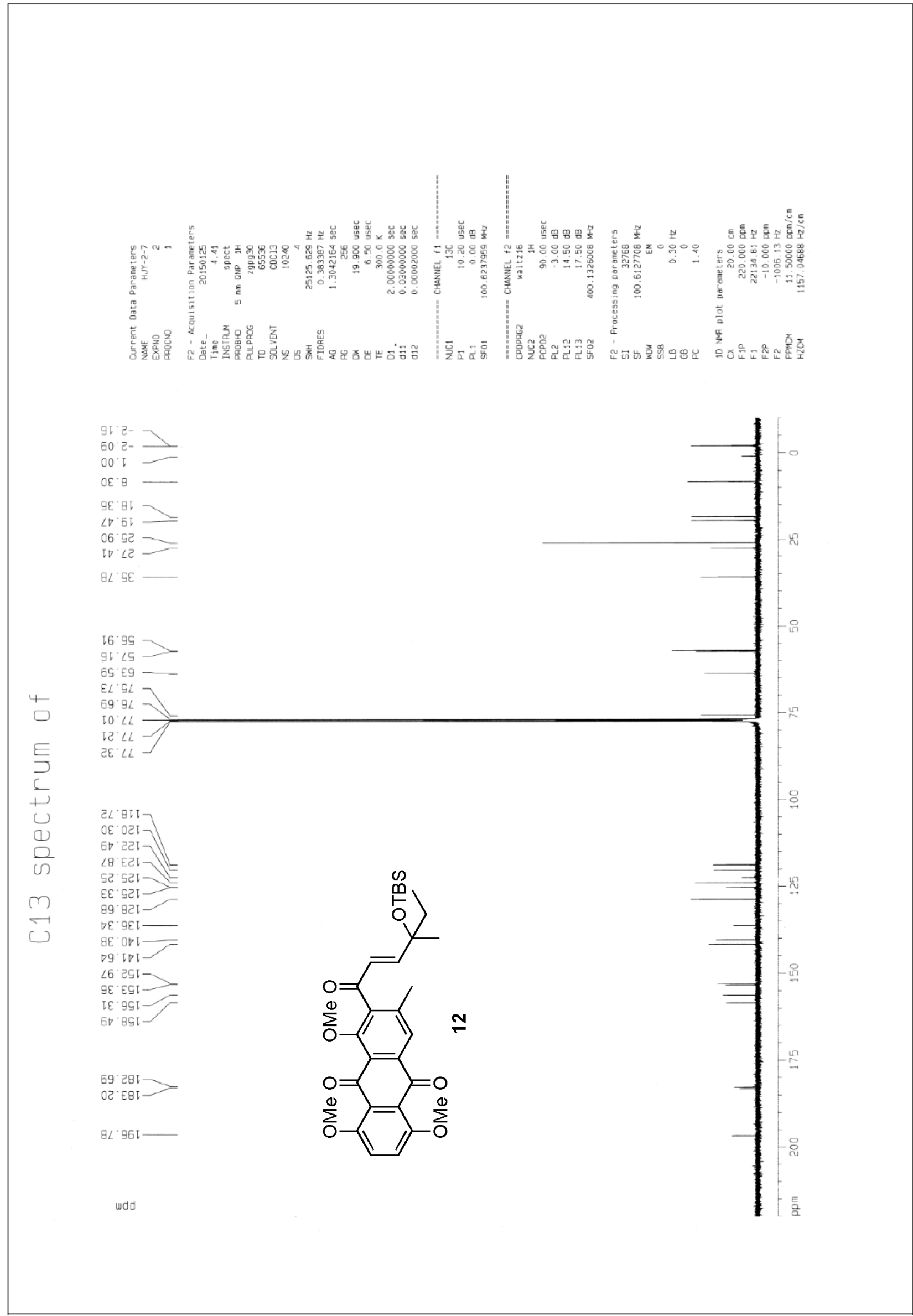




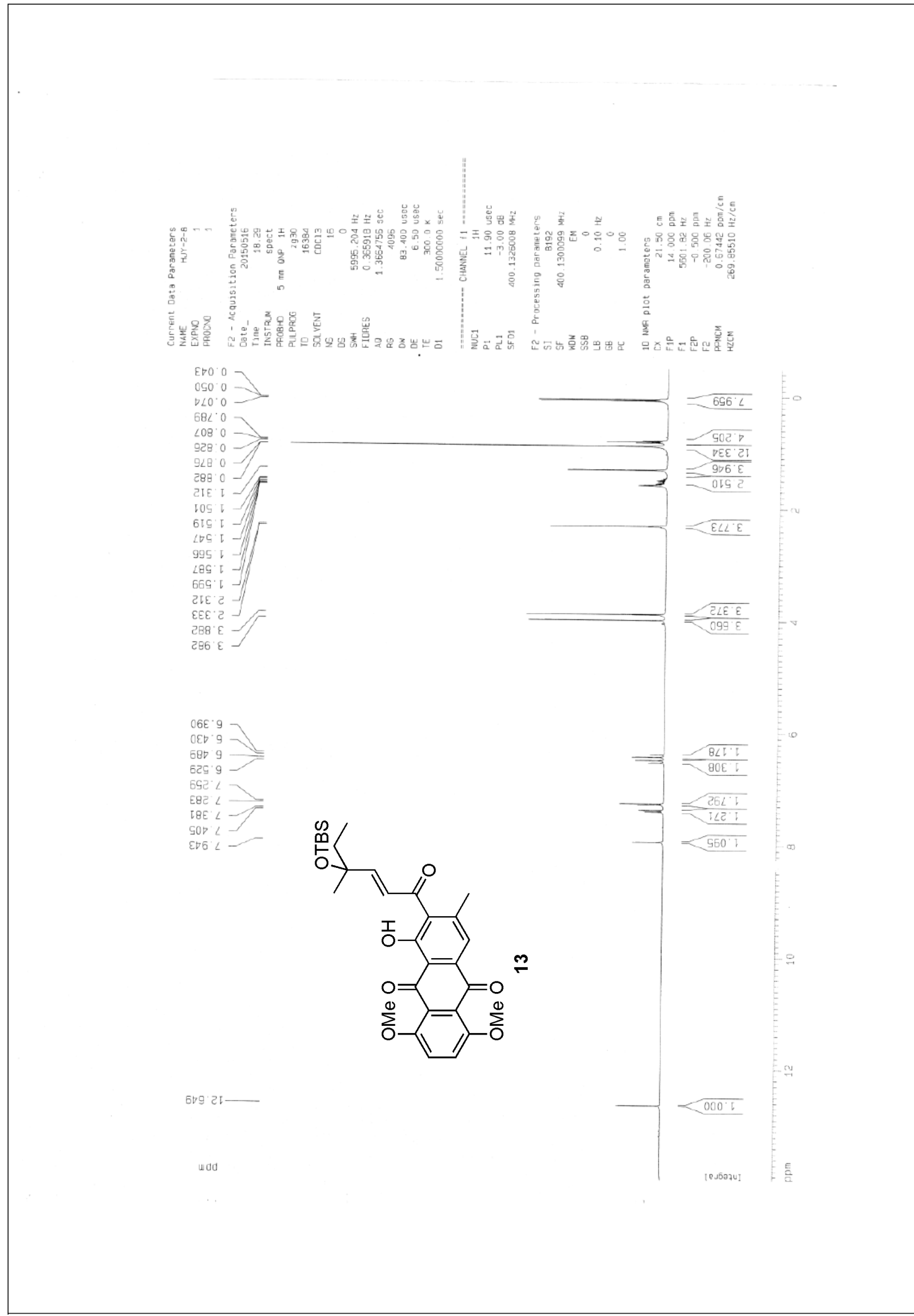



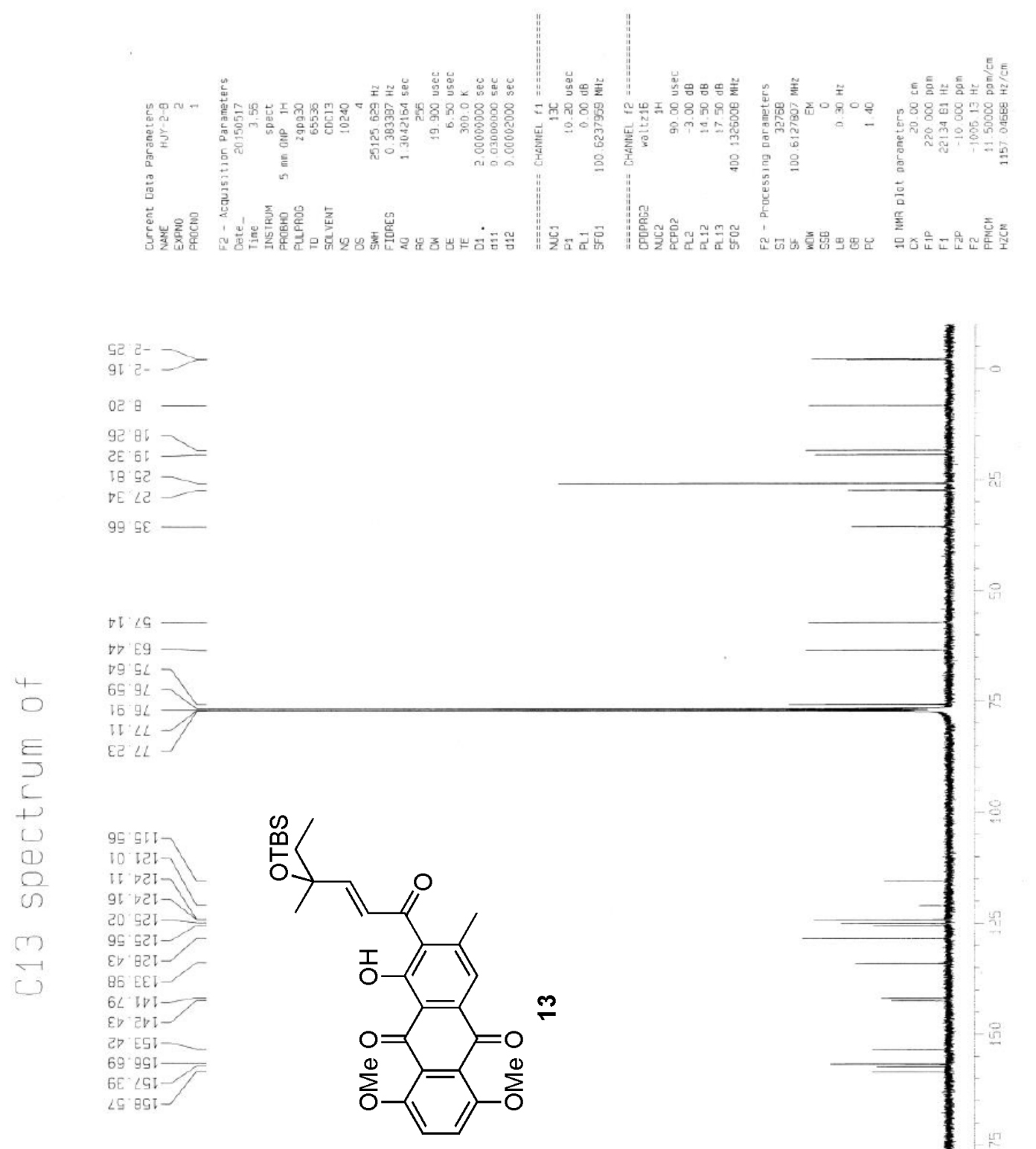

$20 \cdot 581-$
$92 \cdot 881-$
$7 D \cdot 961-$

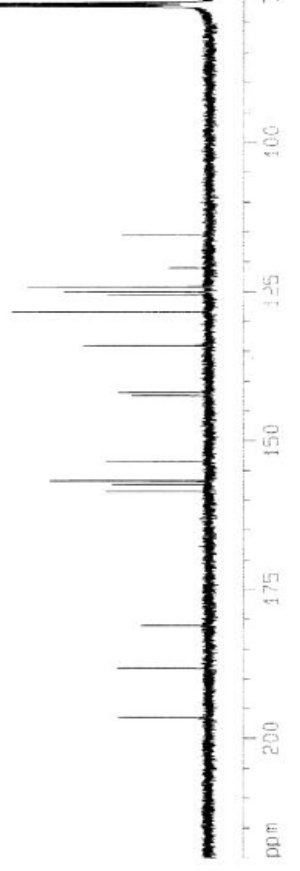




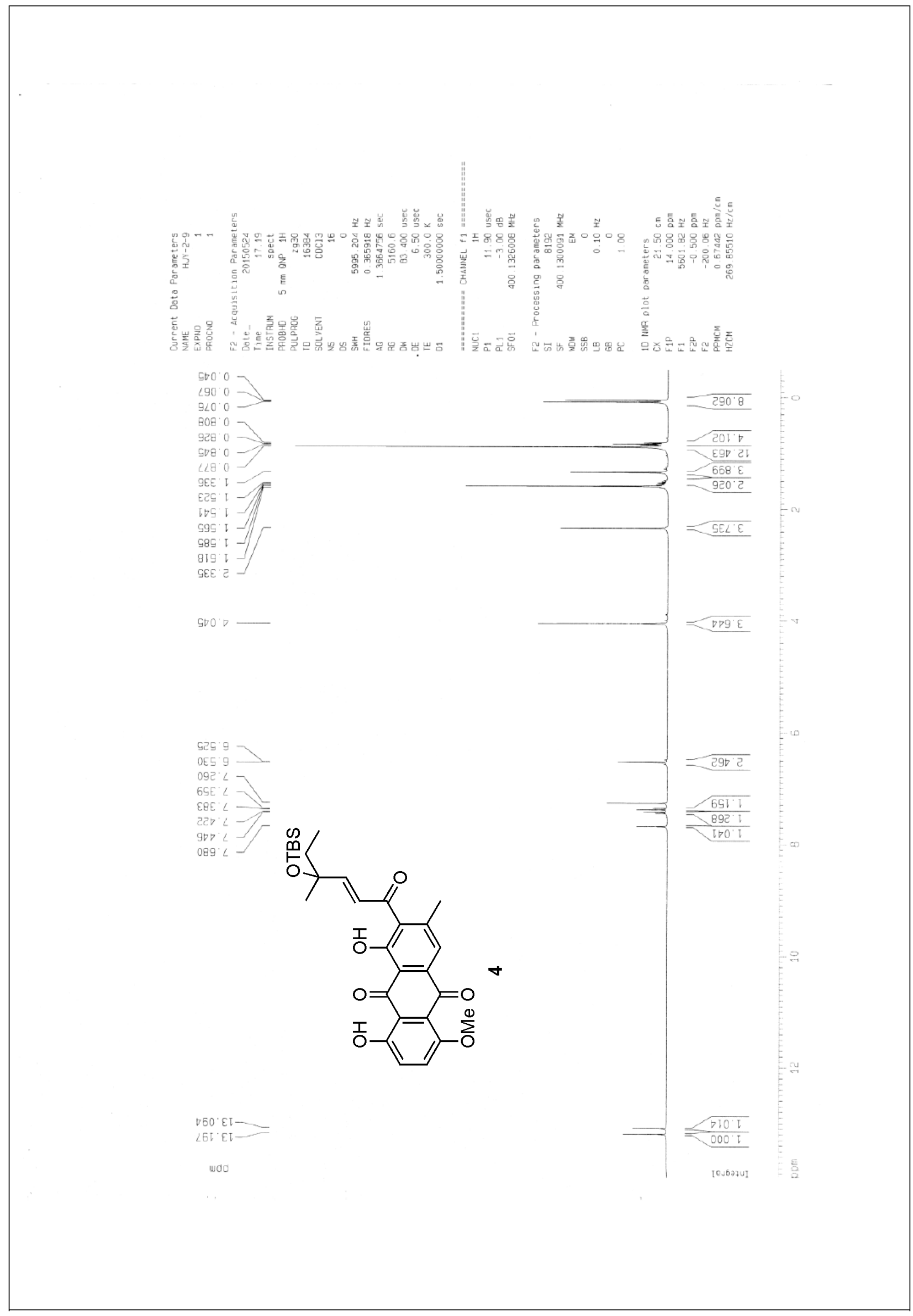




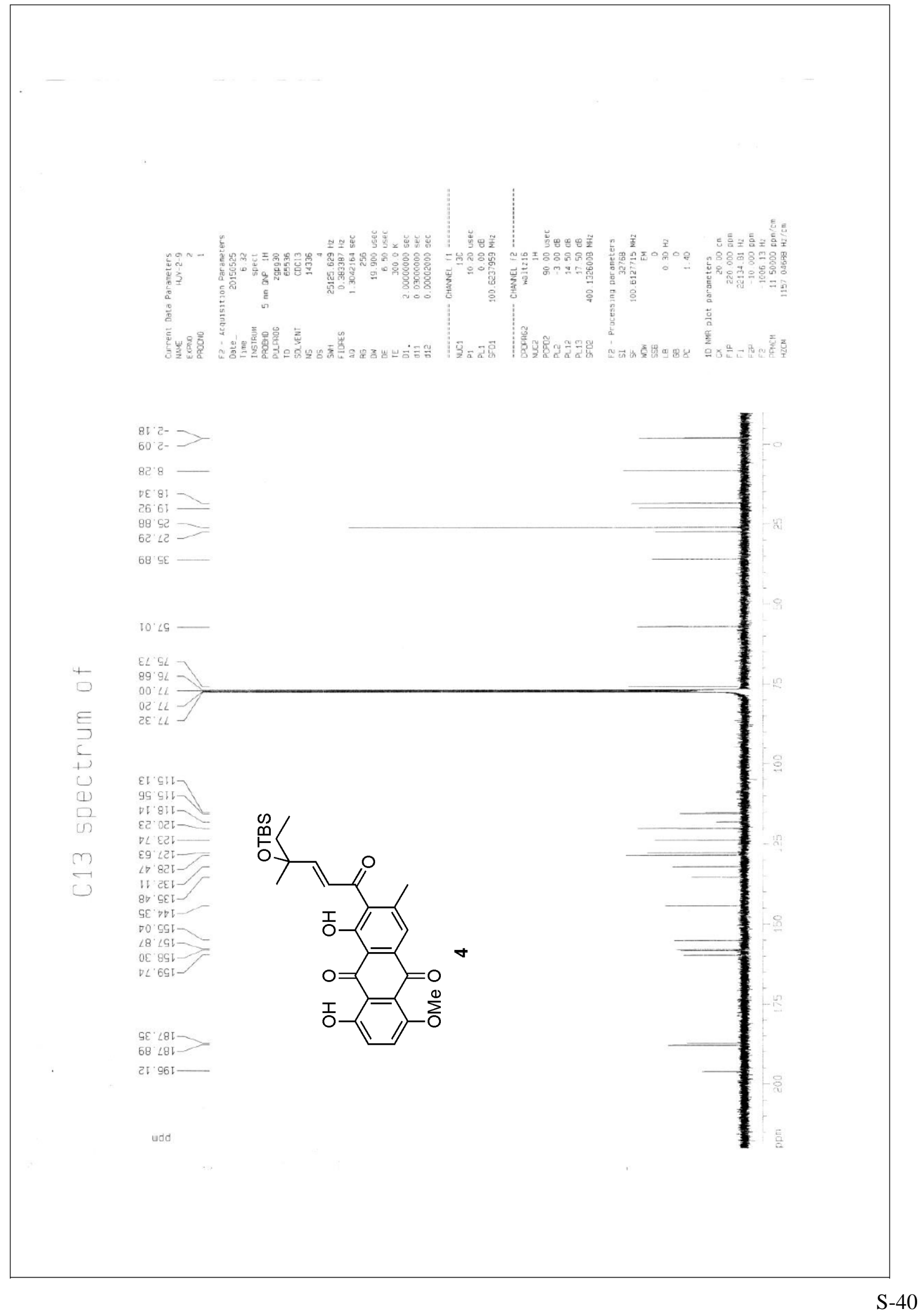




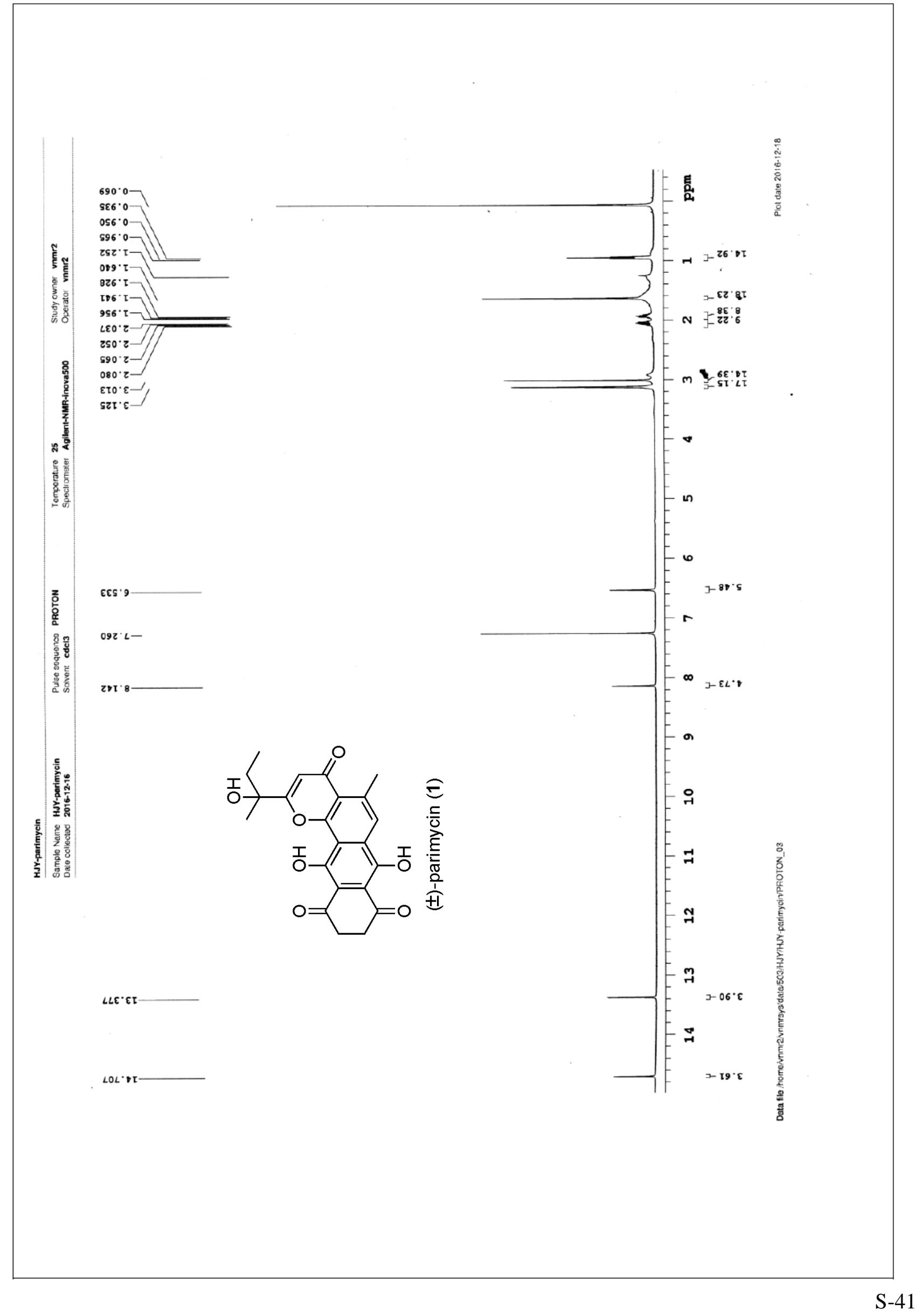




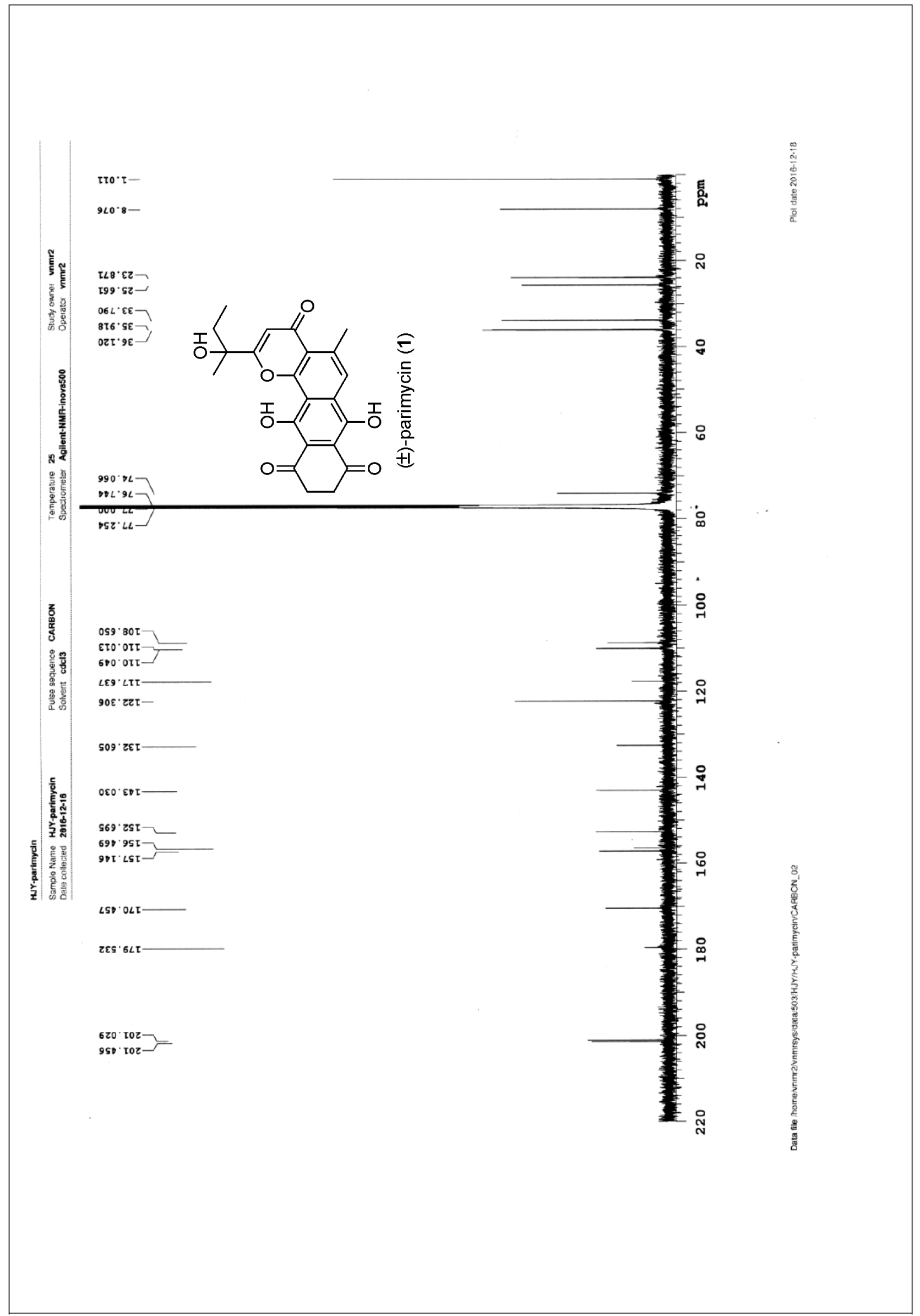




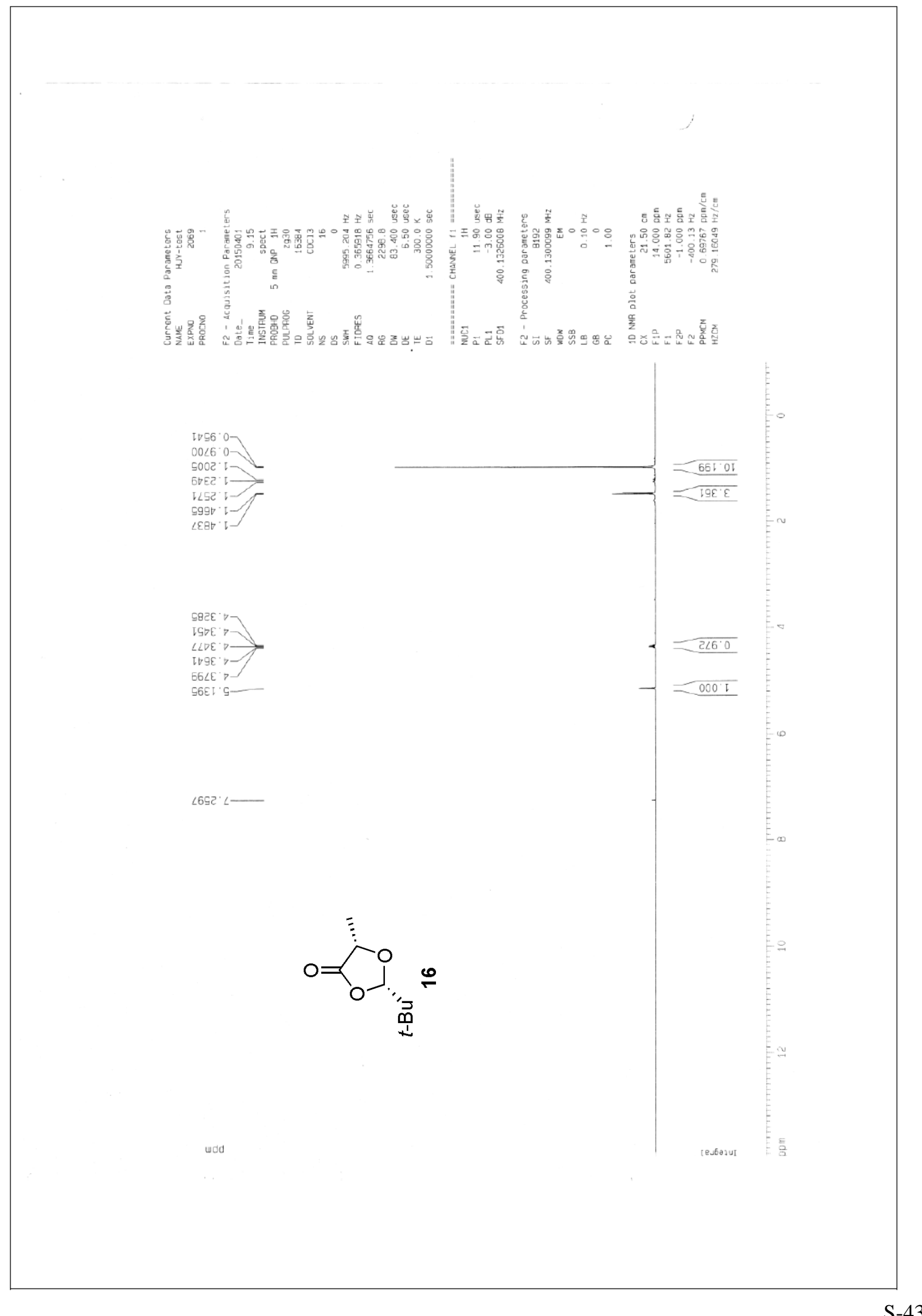




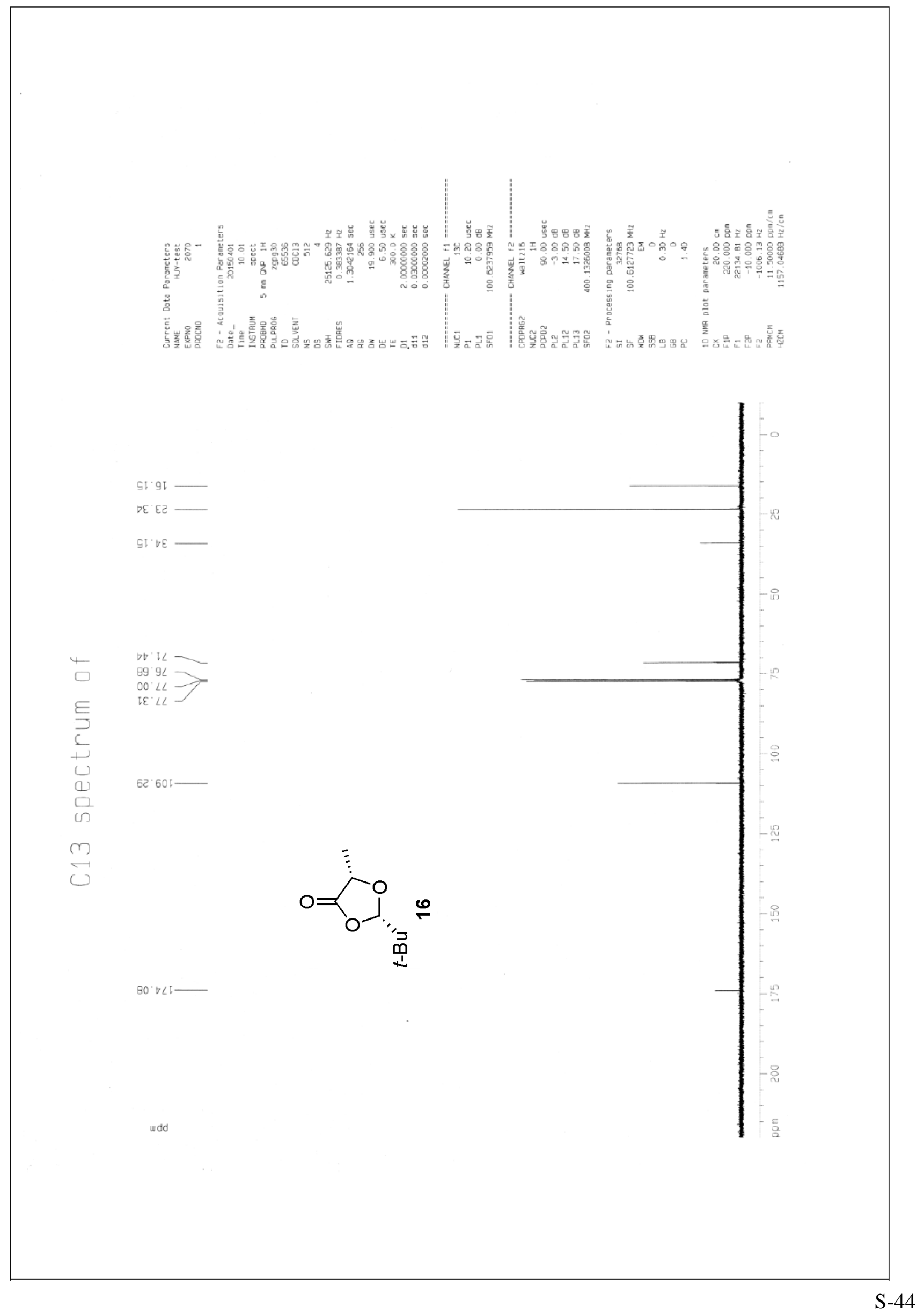




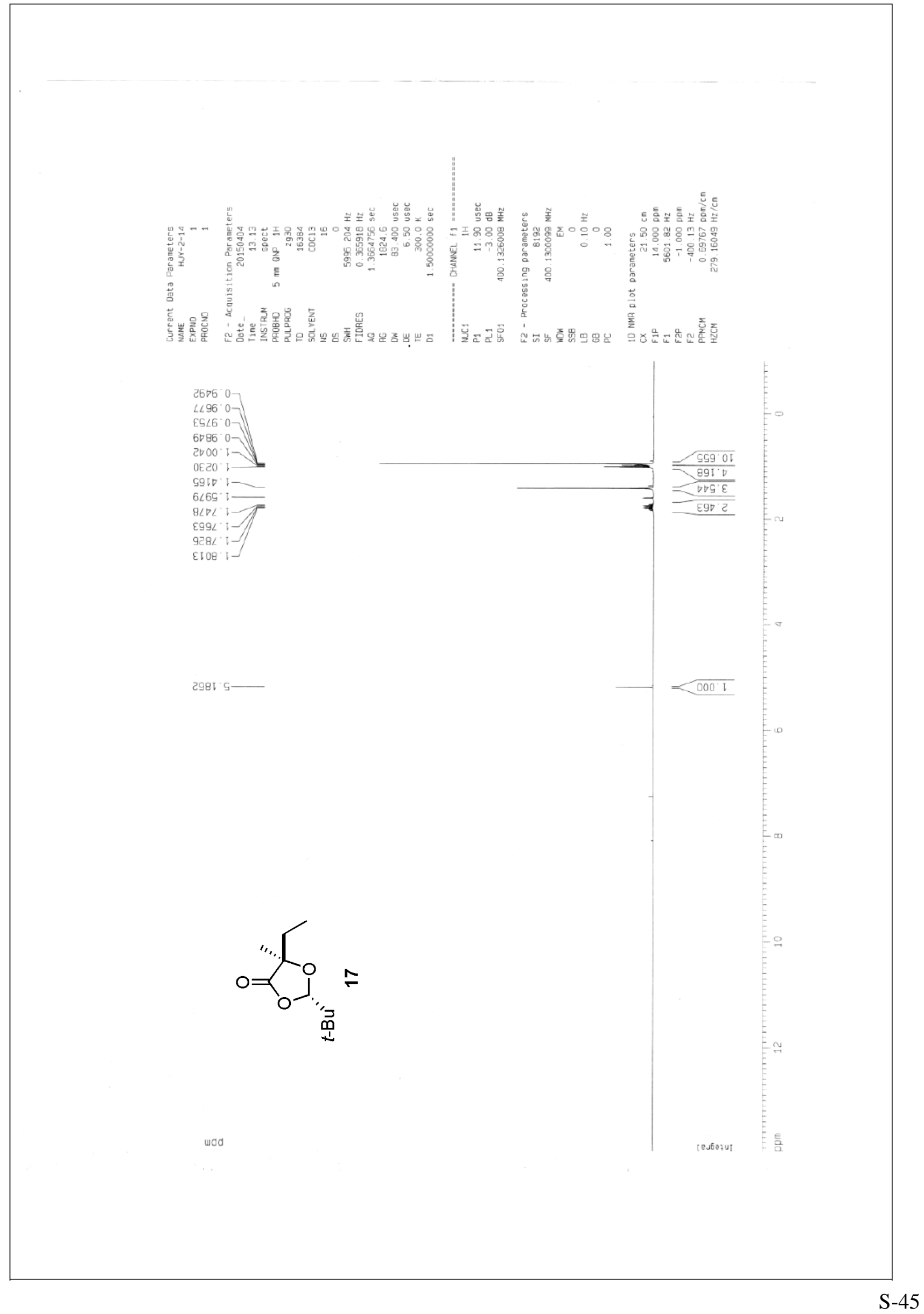




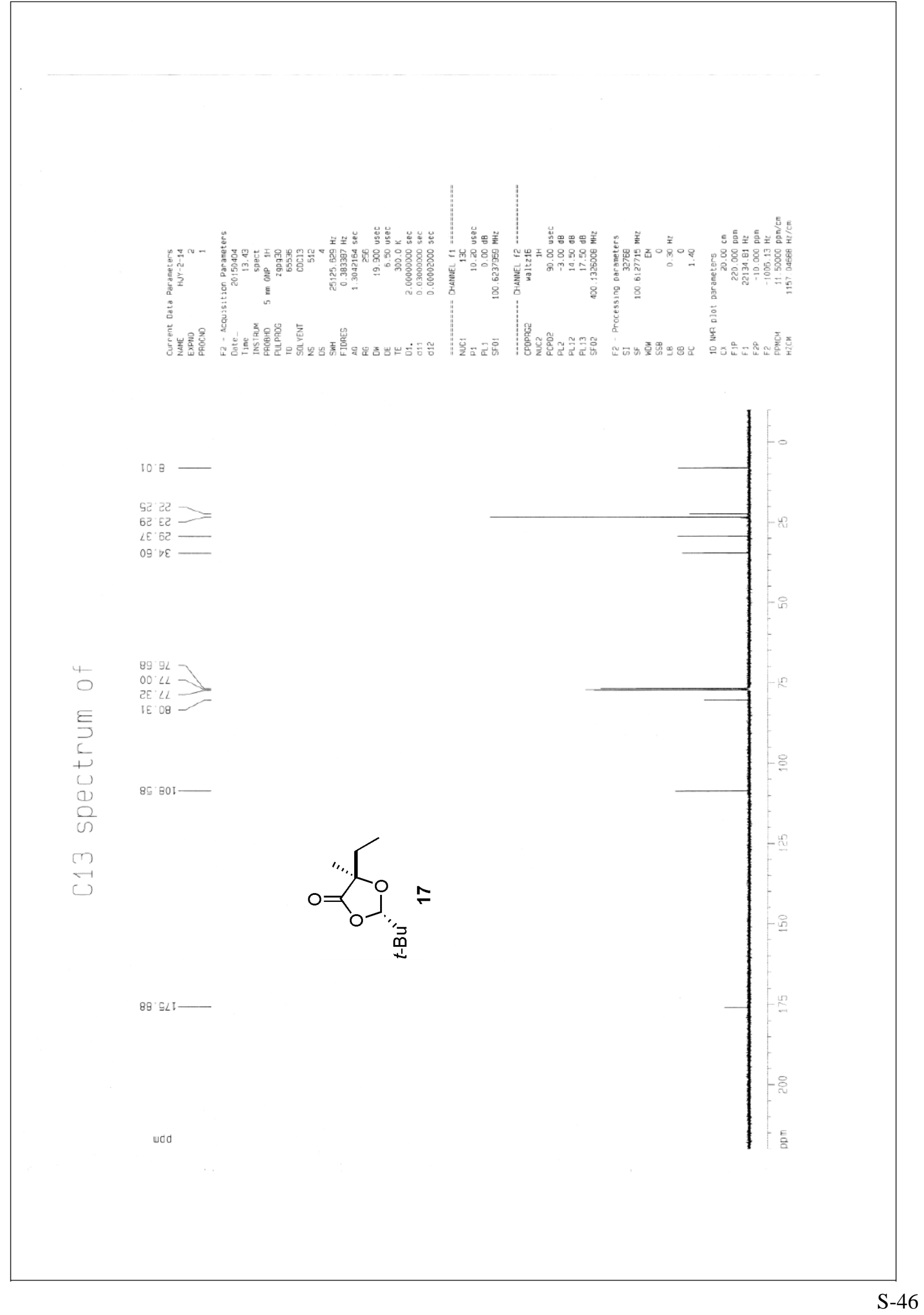




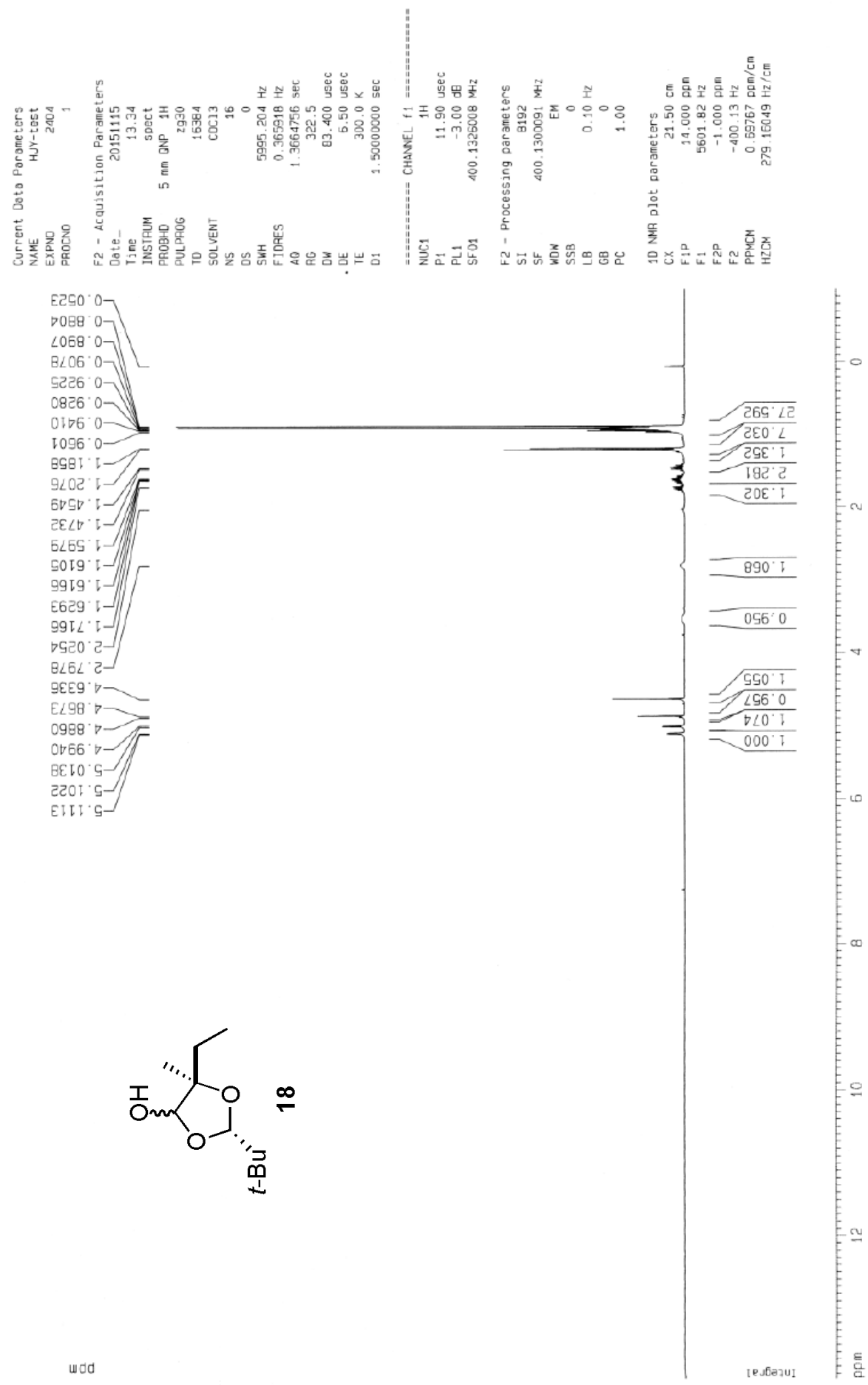



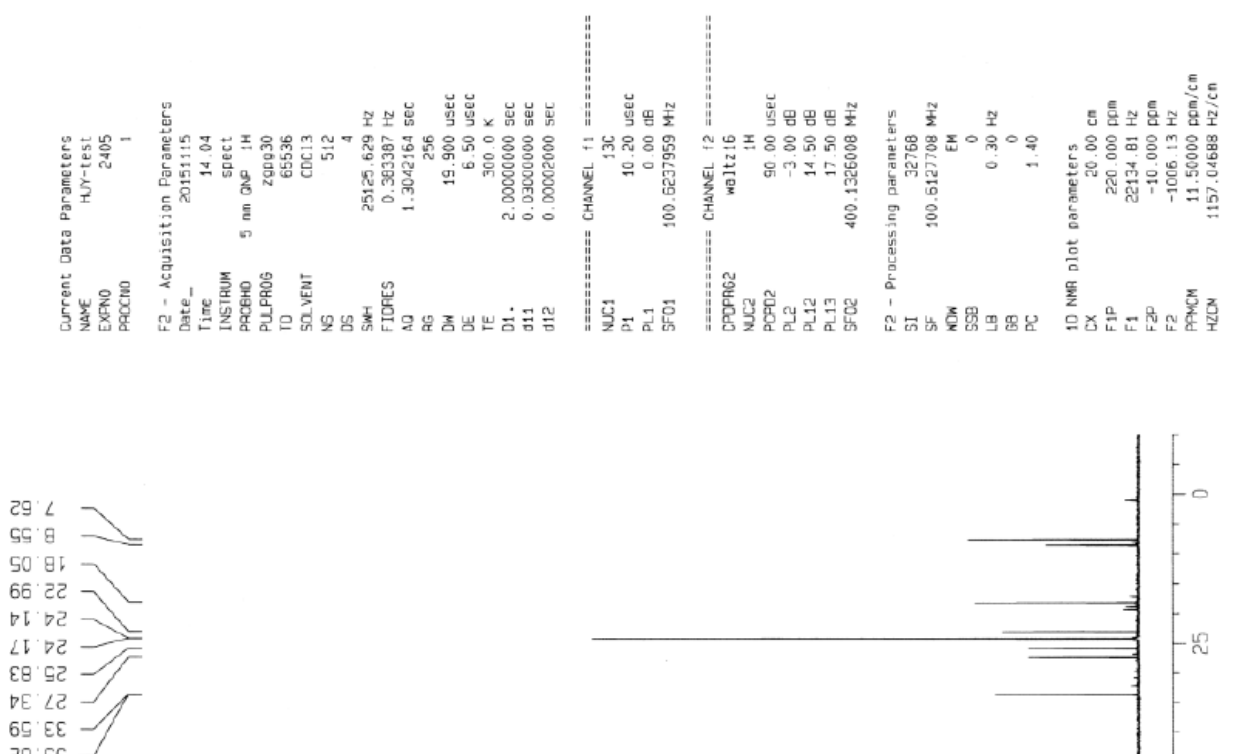

4
6
5
5
0
0
6
6
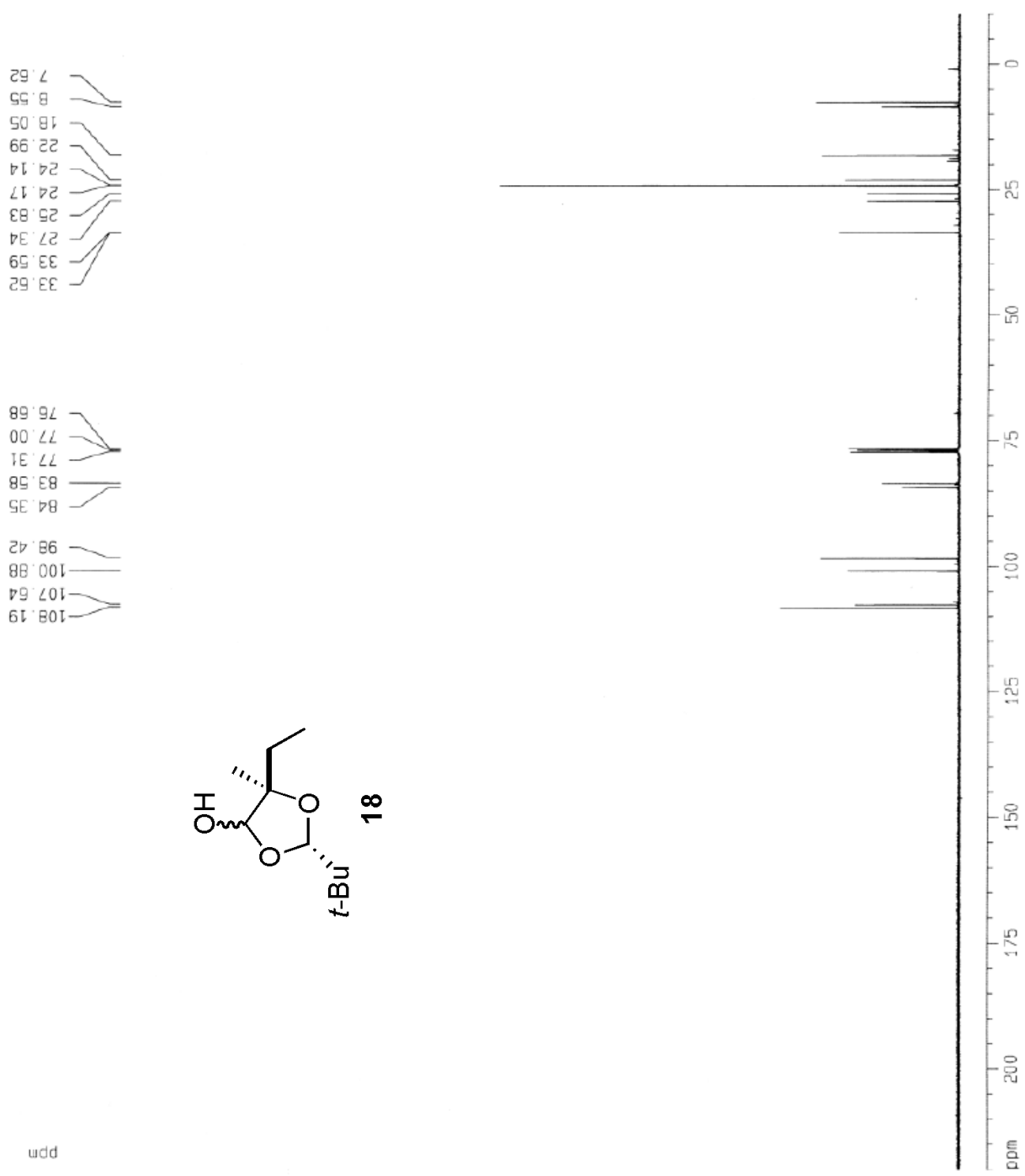

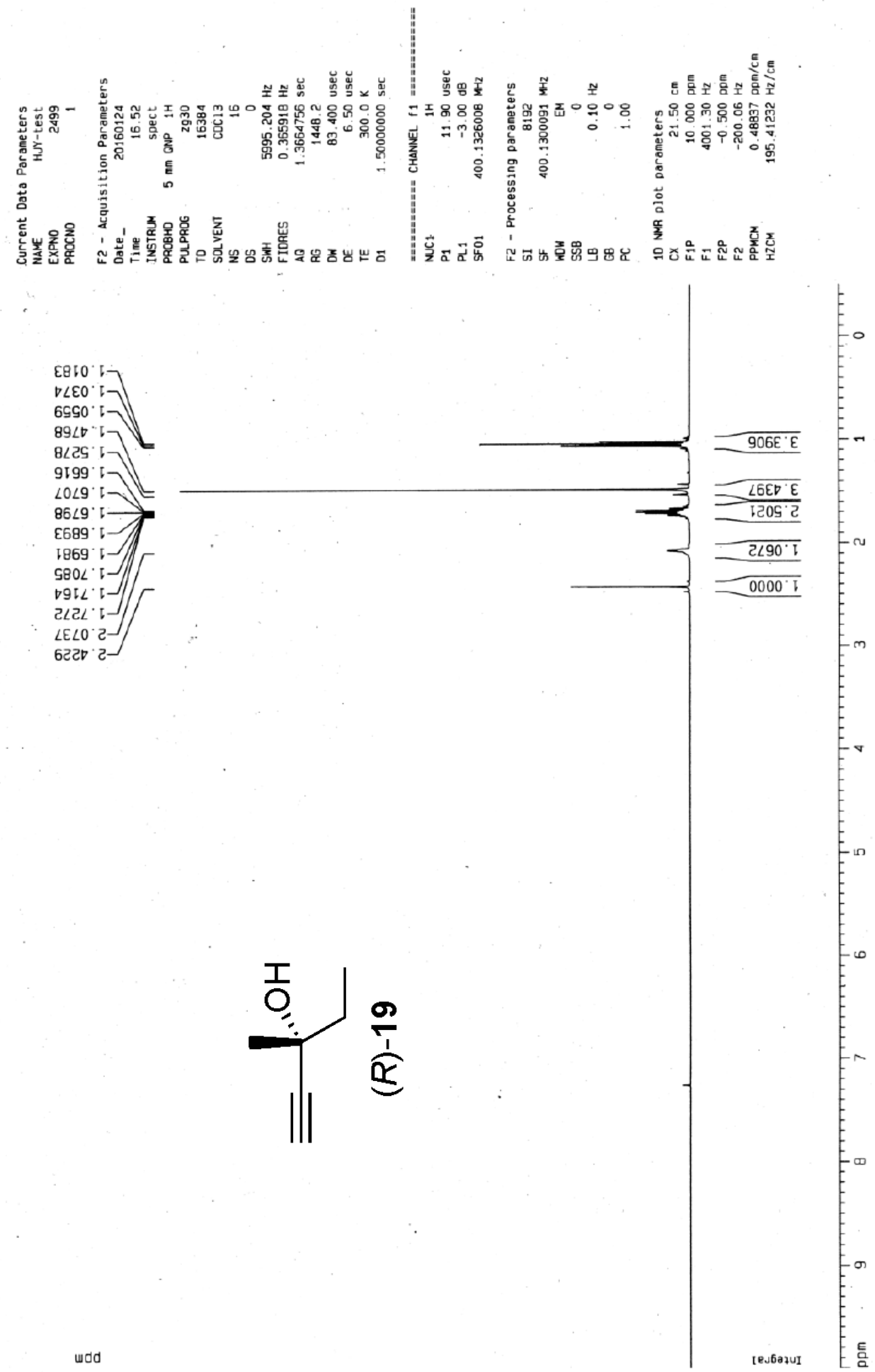


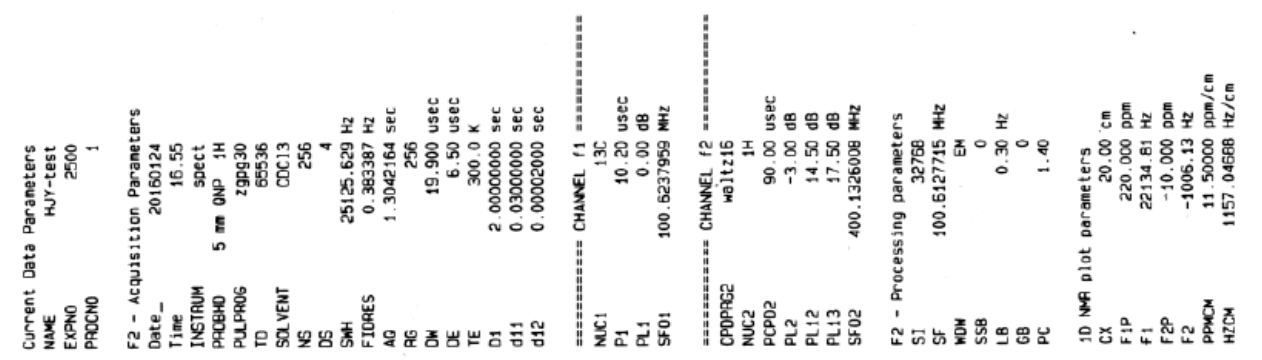

$\angle L L^{\circ} \theta-$

टET' $62-$

५०E' $9 \varepsilon-$

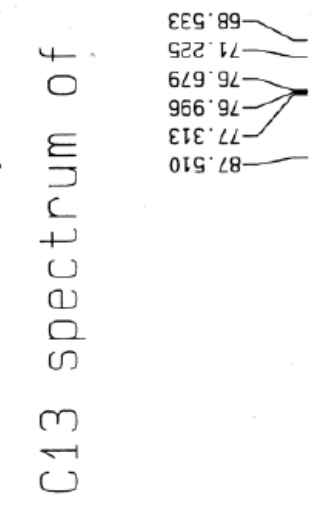

w dd

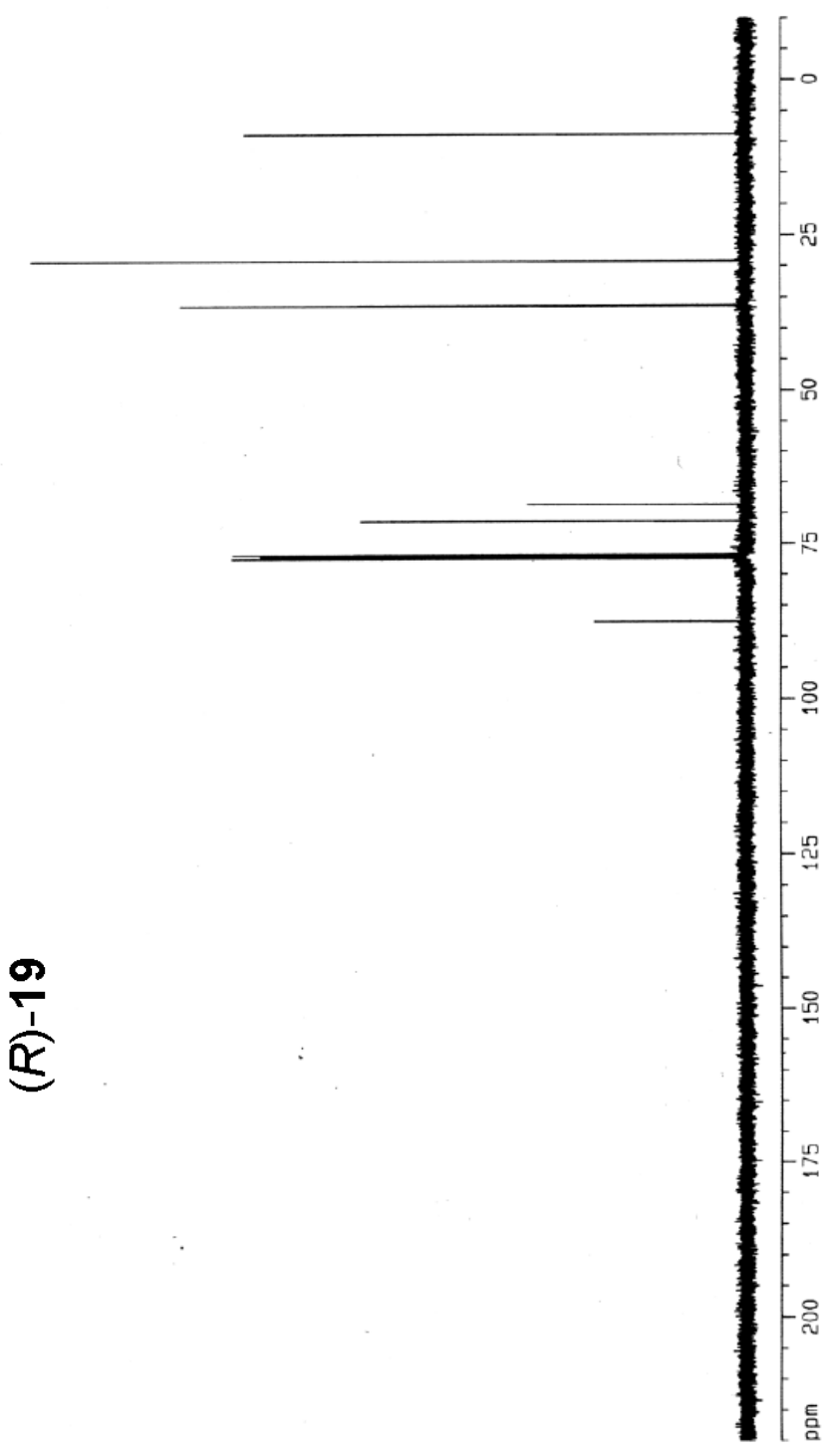




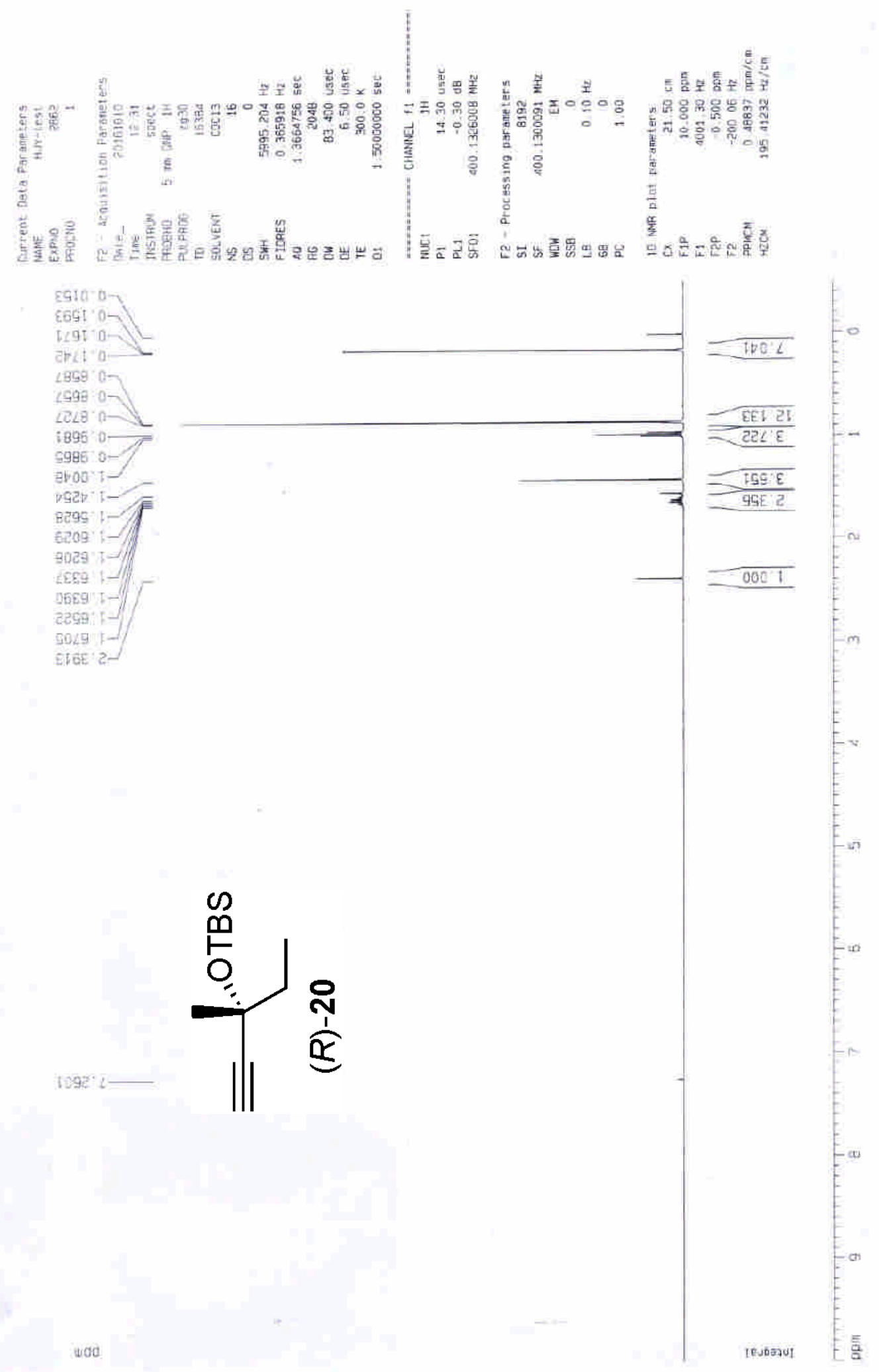




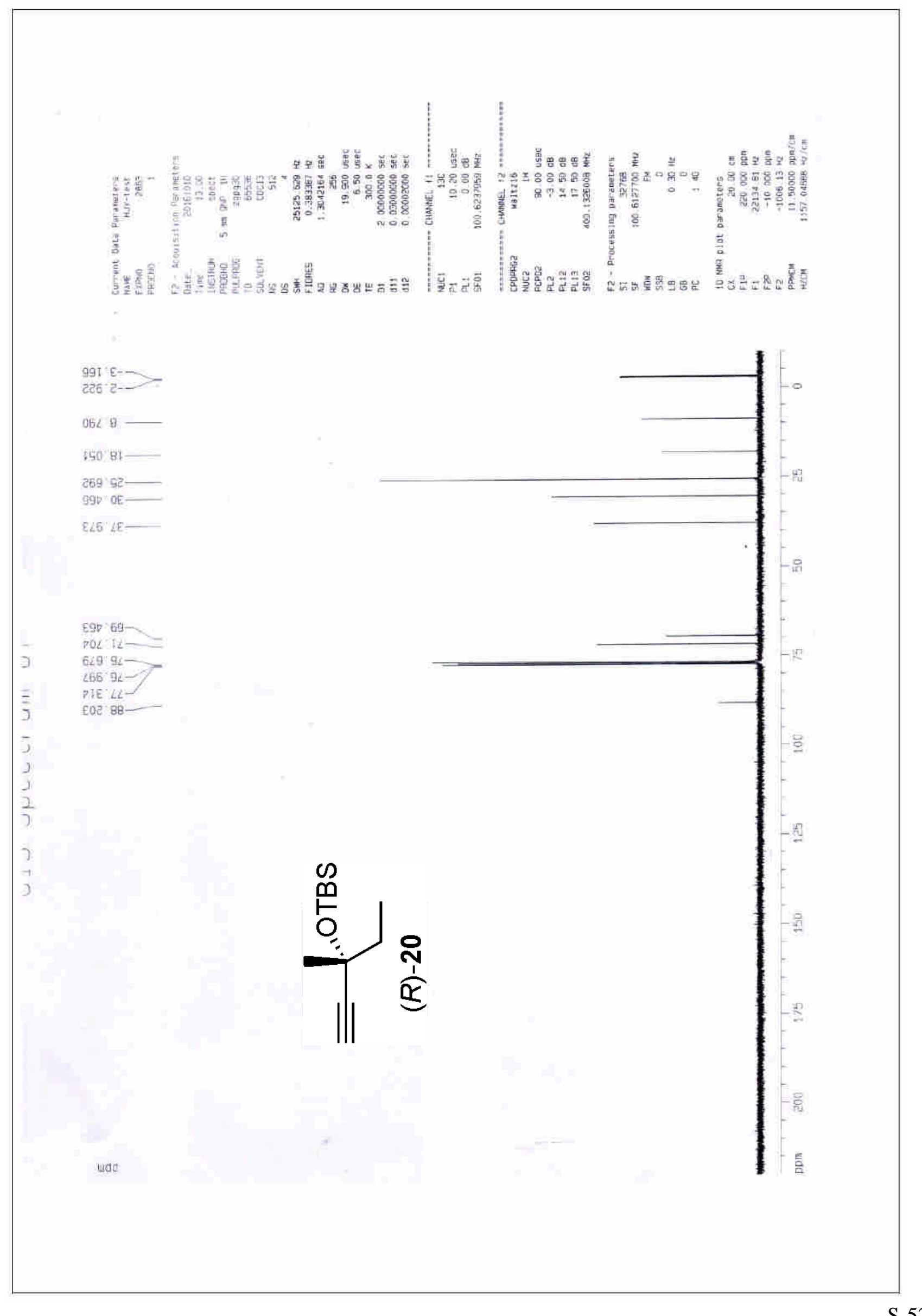




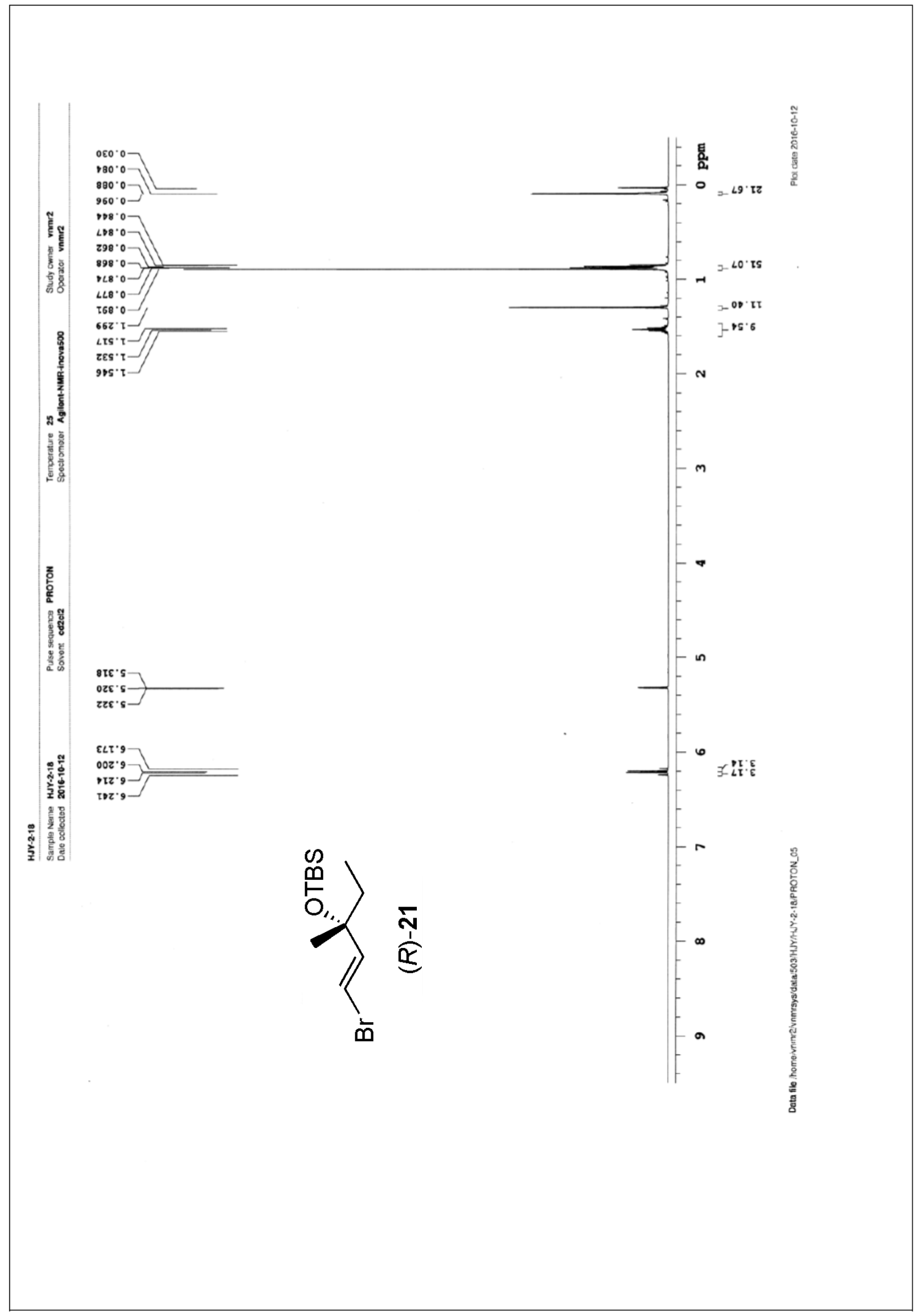




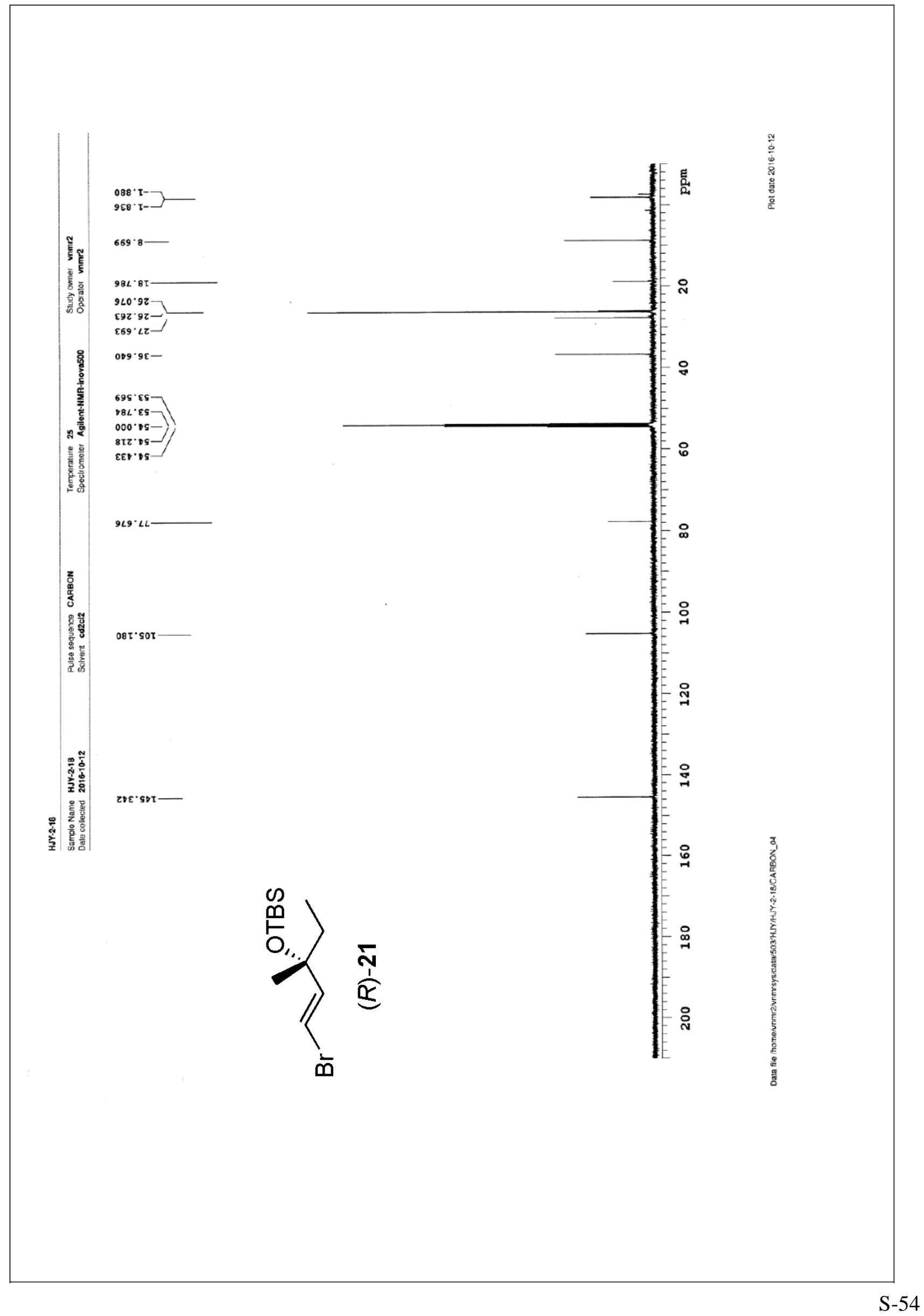




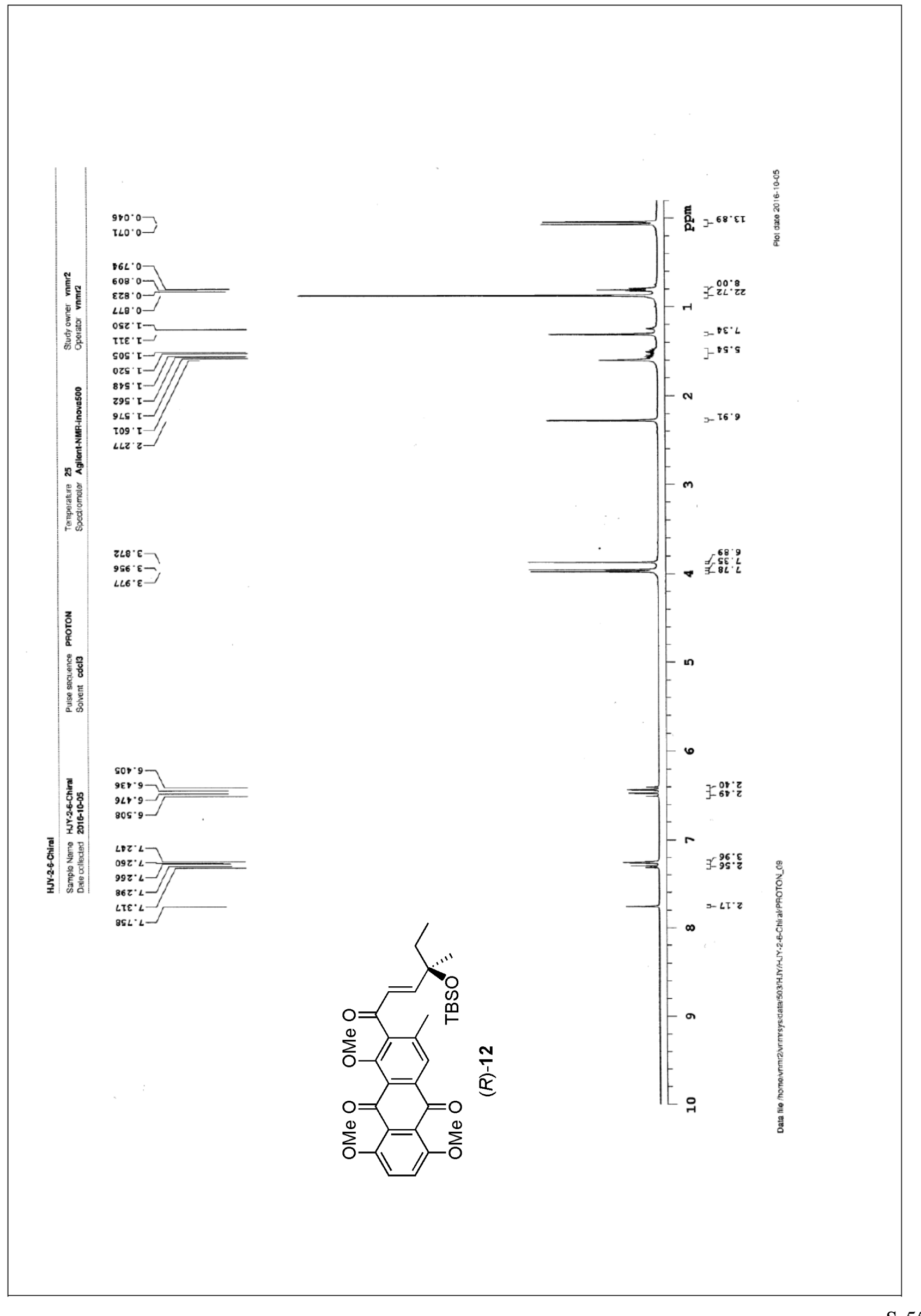




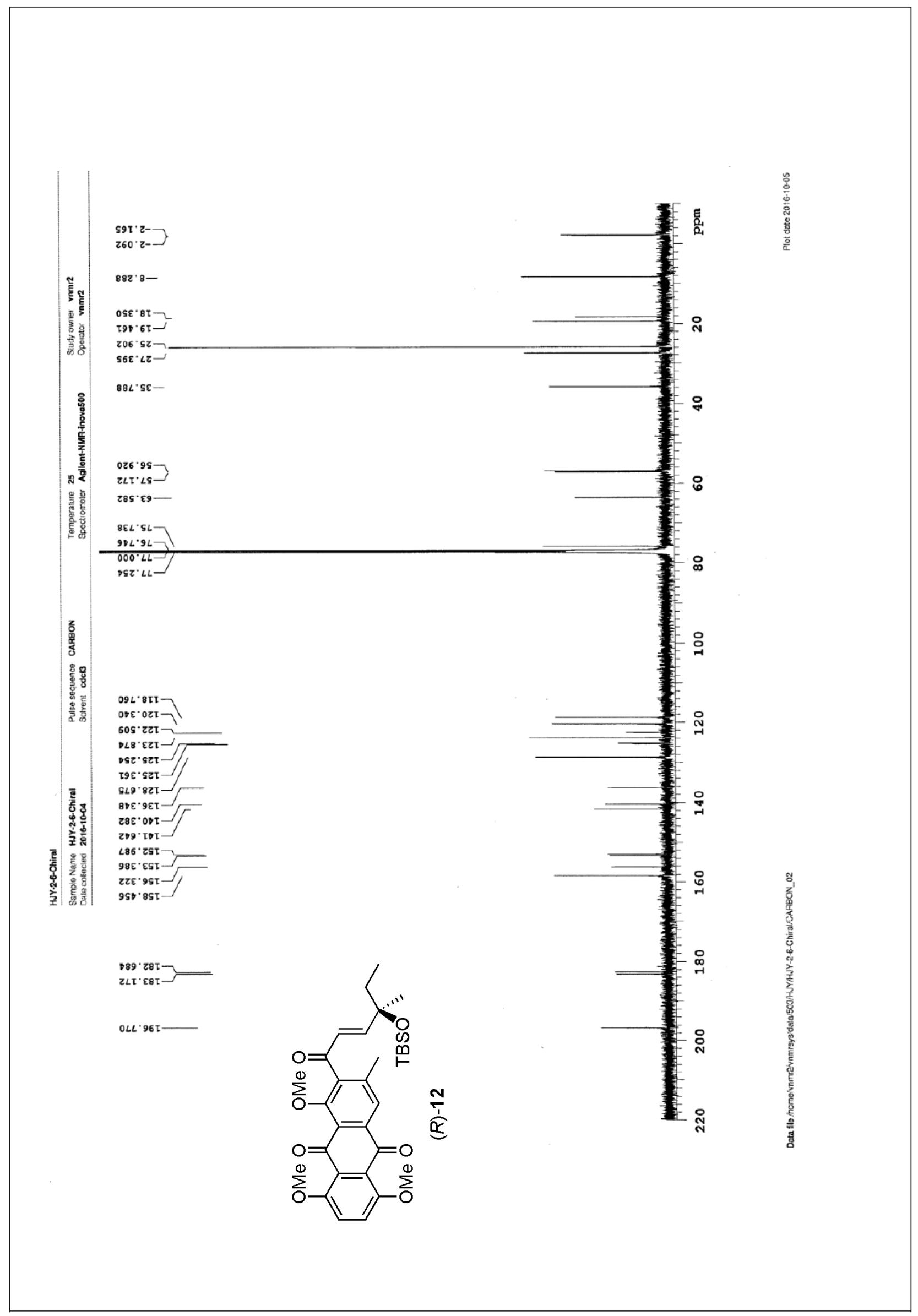




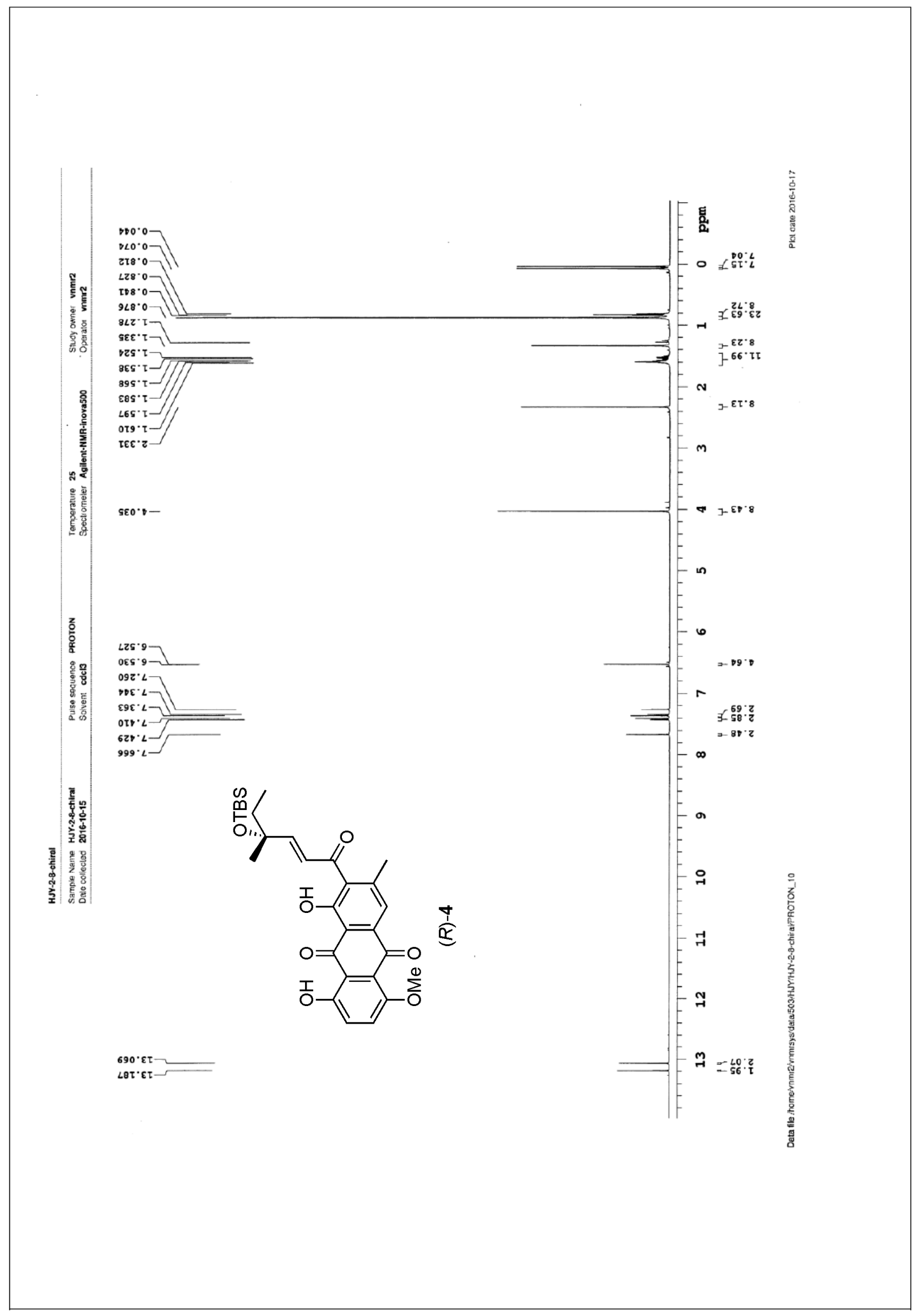




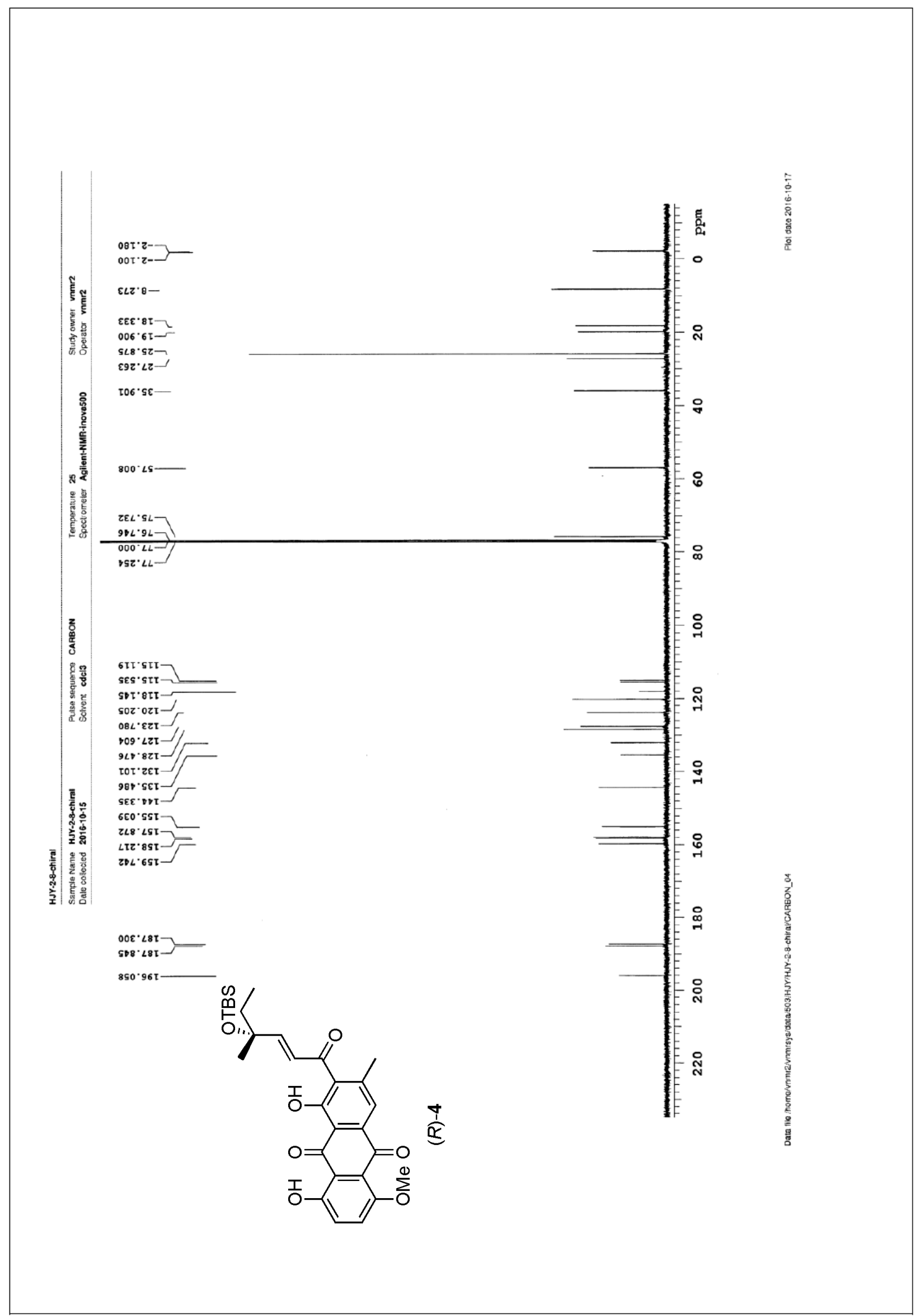




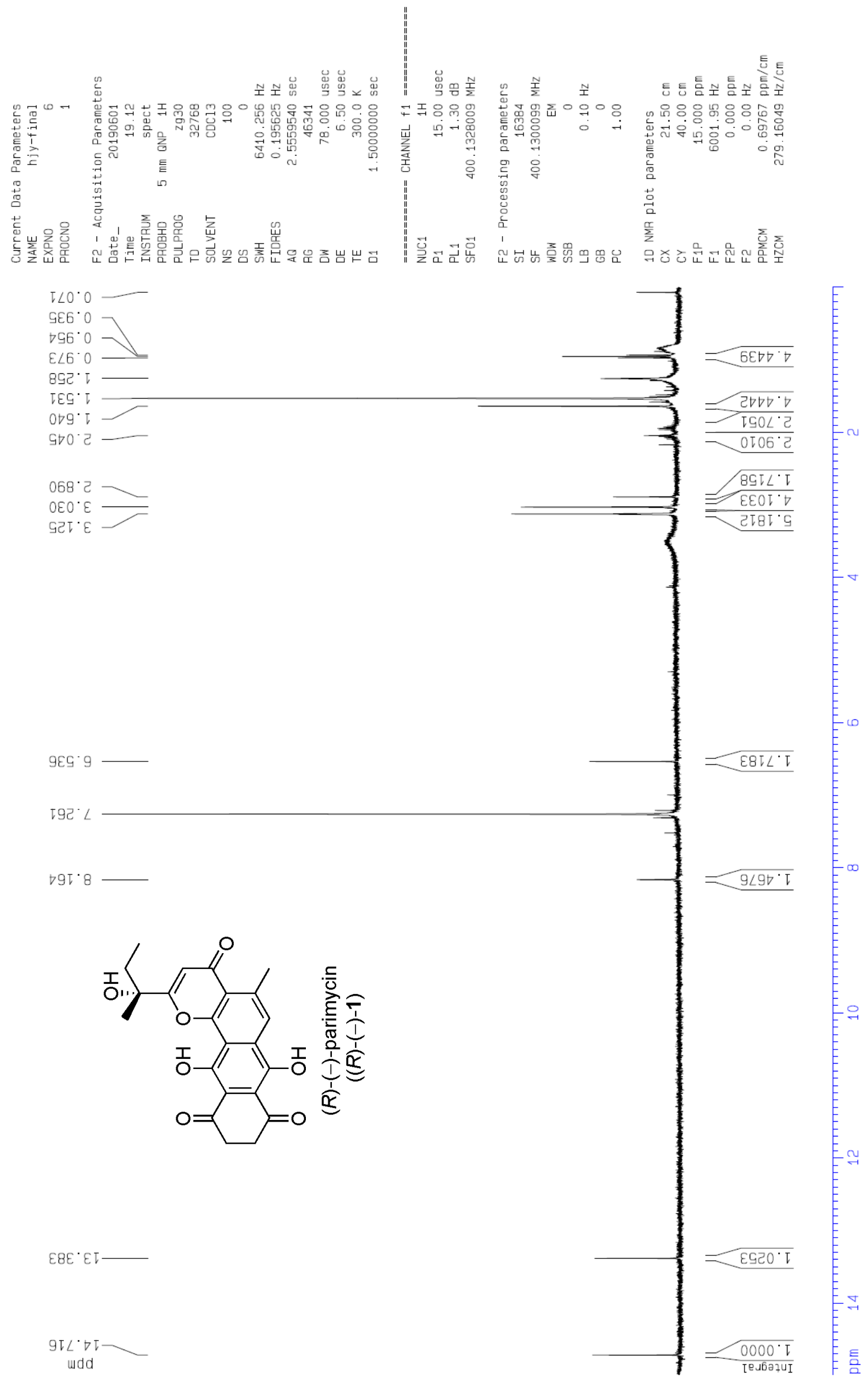




\section{D-2000 Elite HPLC System Manager Report}

Analyzed Date and Time: 10/03/2016

$04: 42 \mathrm{PM}$

Processed Date and Time: 10/03/2016

$$
\text { 05:41 PM }
$$

Data Path: D: \Martin \DATA \0018\

Processing Method: test-EA/Hx

system (acquisition): sys 1

Application (data): Martin

Sample Name: HJY-2-7-rac

Injection from this vial: 1 of 1

Sample Description: 35\% EA/Hx IC
Reported Date and Time: 10/03/2016

$05: 42 \mathrm{PM}$

Series: 0018

Vial Number: 2

Vial Type: UNK

Volume: $20.0 \mathrm{ul}$<smiles>CCC(C)([O-])/C=C/C(=O)c1c(C)cc2c(c1OC)C(=O)c1c(OC)ccc(OC)c1C2=O</smiles>

12

Chrom Type: Fixed WL Chromatogram, $310 \mathrm{~nm}$

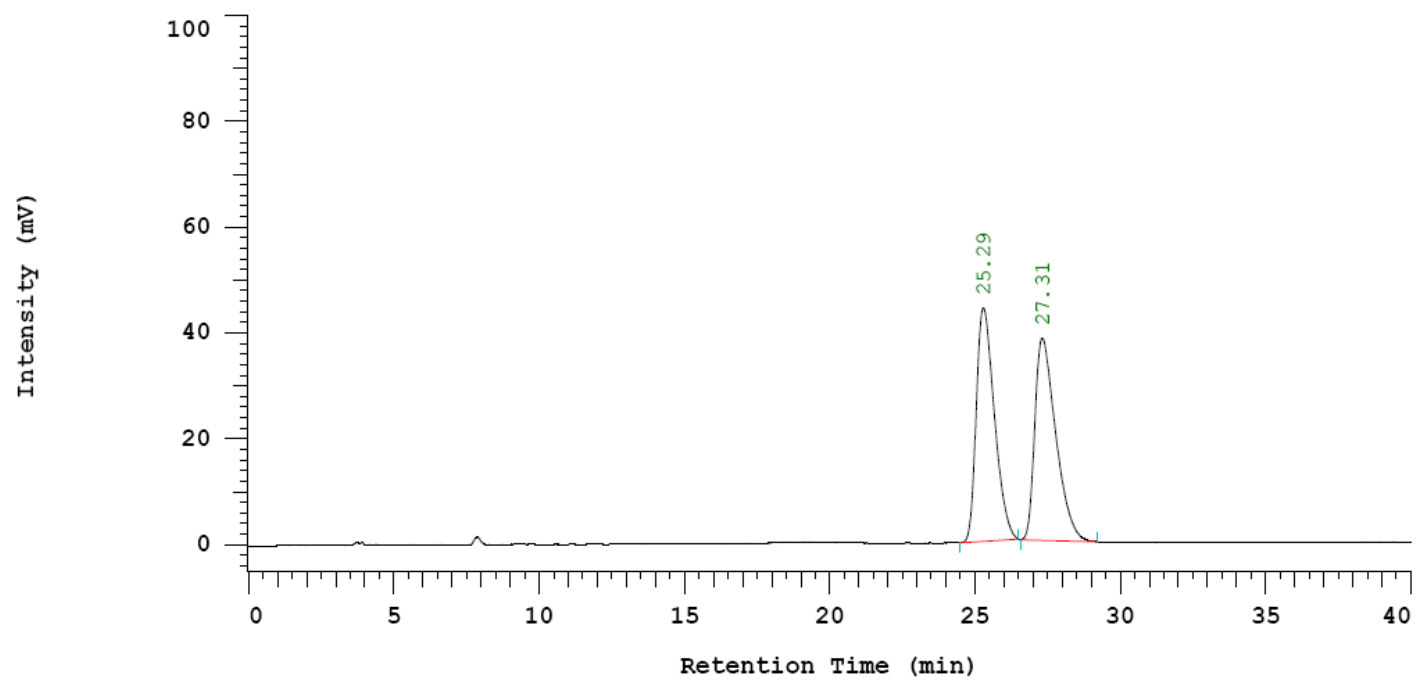

Processing Method: test-EA/Hx

Column Type: IC

Method Developer: Martin

Method Description:

Chrom Type: Fixed WL Chromatogram, $310 \mathrm{~nm}$

Peak Quantitation: AREA

Calculation Method: EXT-STD

scale Factor 1: 1.000

\begin{tabular}{rrrrr} 
No. & RT & Area & Height & Area \\
\hline 1 & 25.29 & 1896535 & 44130 & 49.943 \\
2 & 27.31 & 1900893 & 38153 & 50.057 \\
\hline & & 3797428 & 82283 & 100.000
\end{tabular}

Peak rejection level: 200000 


\section{D-2000 Elite HPLC System Manager Report}

Analyzed Date and Time: 10/03/2016

$04: 01 \mathrm{PM}$

Processed Date and Time: 10/03/2016

05:38 PM

Data Path: D: \Martin $\backslash$ DATA $\backslash 0017 \backslash$

Processing Method: test-EA/Hx

System (acquisition): Sys 1

Application (data): Martin

Sample Name: HJY-2-7-chi

Injection from this vial: 1 of 1

Sample Description: 35\% $\mathrm{EA} / \mathrm{Hx} \mathrm{IC}$
Reported Date and Time: 10/03/2016

$05: 39 \mathrm{PM}$

Series: 0017

Vial Number: 1

Vial Type: UNK

Volume: 20.0 ul<smiles>CC[C@](C)(/C=C/C(=O)c1c(C)cc2c(c1OC)C(=O)c1c(OC)ccc(OC)c1C2=O)O[Na]</smiles>

(R)-12

Chrom Type: Fixed WL Chromatogram, 310 nm

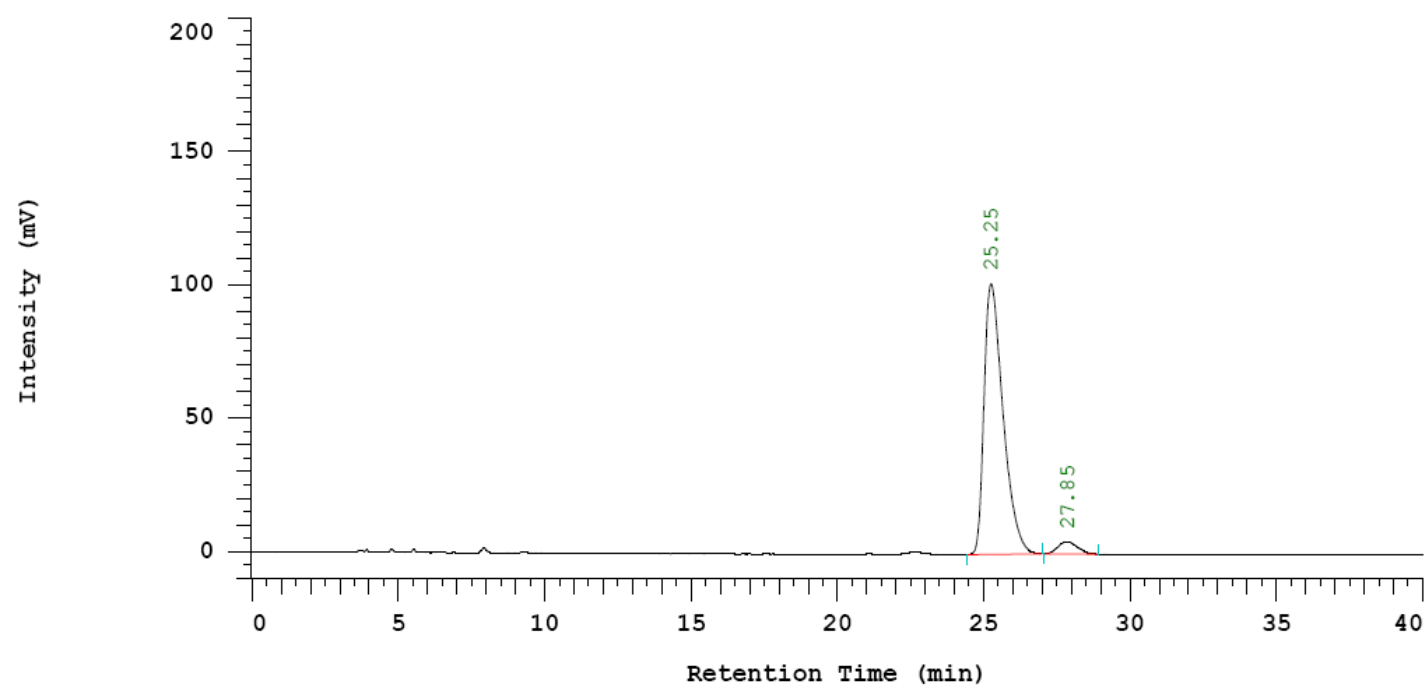

Processing Method: test-EA/Hx

Column Type: IC

Method Developer: Martin

Method Description:

Chrom Type: Fixed WL Chromatogram, $310 \mathrm{~nm}$

Peak Quantitation: AREA

Calculation Method: EXT-STD

Scale Factor 1: 1.000

\begin{tabular}{|c|c|c|c|c|}
\hline No. & $\mathrm{RT}$ & Area & Height & Area 응 \\
\hline 1 & 25.25 & 4538978 & 101290 & 95.444 \\
\hline 2 & 27.85 & 216687 & 4642 & 4.556 \\
\hline & & 4755665 & 105932 & 100.000 \\
\hline
\end{tabular}

Peak rejection level: 200000 


\section{D-2000 Elite HPLC System Manager Report}

Analyzed Date and Time: 10/03/2016 $05: 24 \mathrm{PM}$

Processed Date and Time: 10/03/2016 06:11 PM

Data Path: D: \Martin \DATA \0019\ Processing Method: test-EA/Hx

System (acquisition): sys 1

Application (data): Martin

Sample Name: HJY-2-7-co

Injection from this vial: 1 of 1

Sample Description: 35\% EA/Hx IC

Chrom Type: Fixed WL Chromatogram, $310 \mathrm{~nm}$

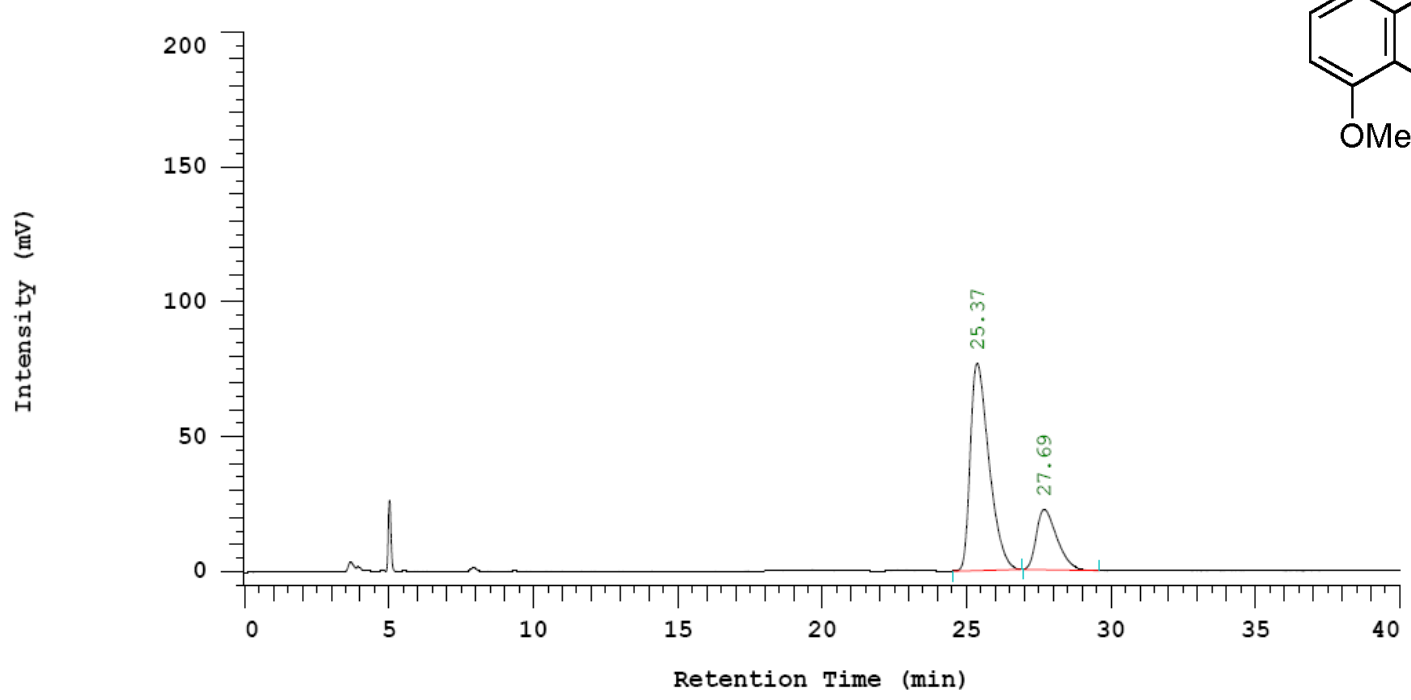

Processing Method: test-EA/Hx

Column Type: IC

Method Developer: Martin

Method Description:

Chrom Type: Fixed WL Chromatogram, $310 \mathrm{~nm}$

Peak Quantitation: AREA

Calculation Method: EXT-STD

scale Factor 1: 1.000

\begin{tabular}{|c|c|c|c|c|}
\hline No. & $\mathrm{RT}$ & Area & Height & Area 응 \\
\hline 1 & 25.37 & 3440292 & 76724 & 75.725 \\
\hline 2 & 27.69 & 1102835 & 22336 & 24.275 \\
\hline & & 4543127 & 99060 & 100.000 \\
\hline
\end{tabular}

Peak rejection level: 200000
: 0019

Vial Number: 3

Vial Type: UNK

Volume: $20.0 \mathrm{ul}$<smiles>CC[C@](C)(/C=C/C(=O)c1c(C)cc2c(c1OC)C(=O)c1c(OC)ccc(OC)c1C2=O)OC</smiles>

(R)-12<smiles></smiles>

12

$+$

TBSO 


\section{D-2000 Elite HPLC System Manager Report}

Analyzed Date and Time: 03/23/2018

$$
01: 42 \mathrm{PM}
$$

Processed Date and Time: 03/27/2018

$$
06: 36 \mathrm{PM}
$$

Data Path: D: \Martin \DATA \0029

Processing Method: test-THF/Hx

System (acquisition): sys 1

Application (data): Martin

Sample Name: HJY-2-parimycin

Injection from this vial: 1 of 1

Sample Description: 18\% $\mathrm{THF} / \mathrm{Hx}$ IC

Chrom Type: Fixed WL Chromatogram, 254 nm
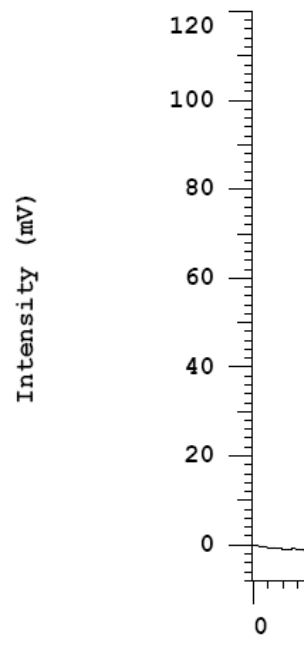

Series: 0029

Vial Number: 1

Vial Type: UNK

Volume: 20.0 ul
$03 / 27 / 2018$

$06: 36 \mathrm{PM}$<smiles>CCC(C)(O)c1cc(=O)c2c(C)cc3c(O)c4c(c(O)c3c2o1)C(=O)CCC4=O</smiles>

$( \pm)$-parimycin (1)

Processing Method: test-THF/Hx

Column Type: IA

Method Developer: Martin

Method Description:

Chrom Type: Fixed WL Chromatogram, $254 \mathrm{~nm}$

Peak Quantitation: AREA

Calculation Method: EXT-STD

Scale Factor 1: 1.000

\begin{tabular}{rrrrr} 
No. & RT & Area & Height & Area \% \\
\hline 1 & 65.14 & 3339483 & 23803 & 49.221 \\
2 & 72.21 & 3445123 & 22166 & 50.779 \\
\hline & & 6784606 & 45969 & 100.000
\end{tabular}

Peak rejection level: 200000 


\section{D-2000 Elite HPLC System Manager Report}

Analyzed Date and Time: 03/23/2018 $03: 48 \mathrm{PM}$

Processed Date and Time: 03/27/2018 $06: 39 \mathrm{PM}$

Data Path: D: \Martin $\backslash$ DATA $\backslash 0030 \backslash$

Processing Method: test-THF/Hx

System (acquisition): Sys 1

Application(data): Martin

Sample Name: HJY-2-parimycin-chiral

Injection from this vial: 1 of 1

Sample Description: 18\% $\mathrm{THF} / \mathrm{Hx}$ IC

Chrom Type: Fixed WL Chromatogram, 254 nm

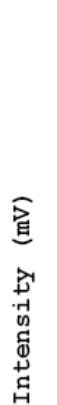

Reported Date and Time: 03/27/2018

$06: 39 \mathrm{PM}$

Series: 0030

Vial Number: 1

Vial Type: UNK

Volume: 20.0 ul<smiles>CC[C@@](C)(O)c1cc(=O)c2c(C)cc3c(O)c4c(c(O)c3c2o1)C(=O)CCC4=O</smiles>

$(R)-(-)$-parimycin $((R)-(-)-1)$

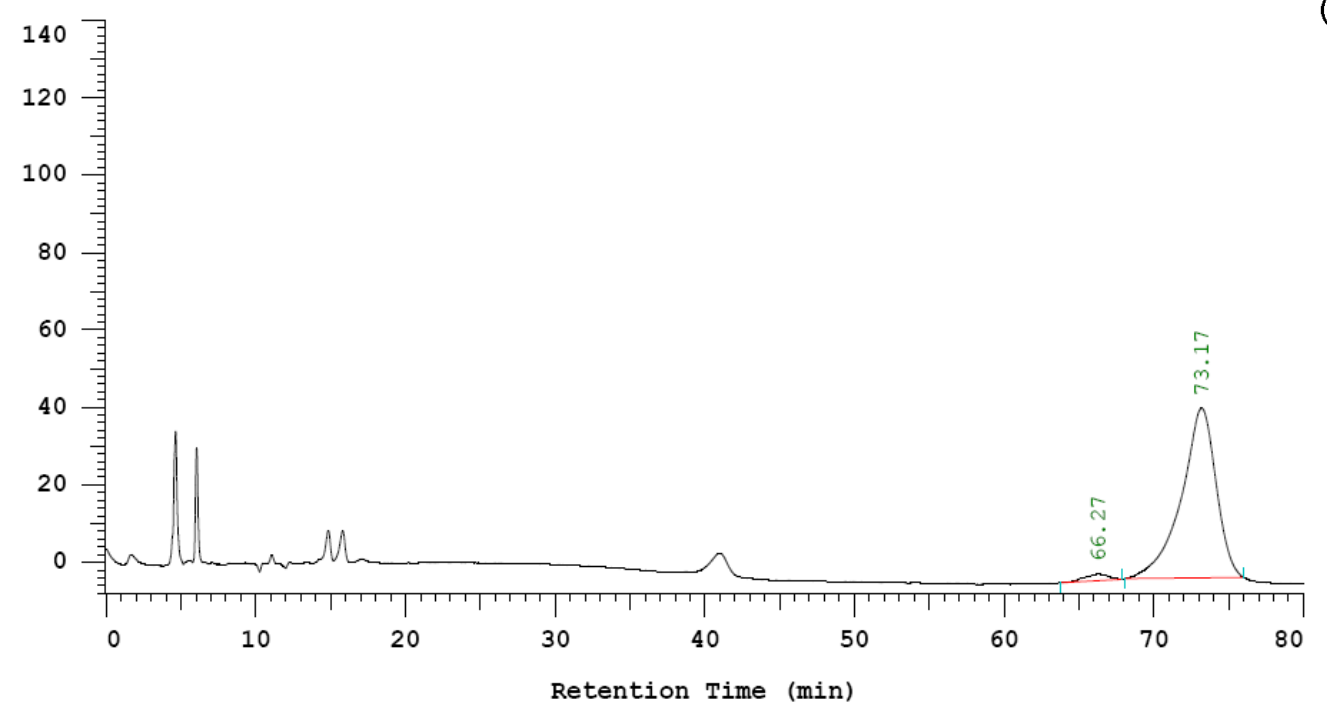

Processing Method: test-THF/Hx

Column Type: IC

Method Developer: Martin

Method Description:

Chrom Type: Fixed WL Chromatogram, $254 \mathrm{~nm}$

Peak Quantitation: AREA

Calculation Method: EXT-STD

Scale Factor 1: 1.000

\begin{tabular}{|c|c|c|c|c|}
\hline No. & $\mathrm{RT}$ & Area & Height & Area 응 \\
\hline 1 & 66.27 & 184567 & 1614 & 2.561 \\
\hline 2 & 73.17 & 7022218 & 43735 & 97.439 \\
\hline & & 7206785 & 45349 & 100.000 \\
\hline
\end{tabular}

Peak rejection level: 1000 


\section{D-2000 Elite HPLC System Manager Report}

Analyzed Date and Time: 03/23/2018 $05: 38 \mathrm{PM}$

Processed Date and Time: 03/27/2018

$06: 42 \mathrm{PM}$

Data Path: D: \Martin \DATA \0031

Processing Method: test-THF/Hx

system (acquisition): sys 1

Application(data): Martin

Sample Name: HJY-2-parimycin-chiral

Injection from this vial: 1 of 1

Sample Description: 18\% $\mathrm{THF} / \mathrm{Hx}$ IC

Chrom Type: Fixed WL Chromatogram, 254 nm



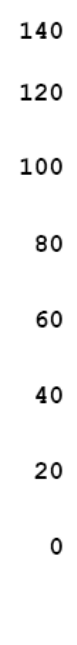

Reported Date and Time: $03 / 27 / 2018$ $06: 43 \mathrm{PM}$

Series: 0031

Vial Number: 1

Vial Type: UNK

Volume: 20.0 ul<smiles>CCC(C)(O)c1cc(=O)c2c(C)cc3c(O)c4c(c(O)c3c2o1)C(=O)CCC4=O</smiles>

$( \pm)$-parimycin (1)<smiles>CC[C@@](C)(O)c1cc(=O)c2c(C)cc3c(O)c4c(c(O)c3c2o1)C(=O)CCC4=O</smiles>

$(R)-(-)$-parimycin $((R)-(-)-1)$

Processing Method: test-THF/Hx

Column Type: IC

Method Developer: Martin

Method Description:

Chrom Type: Fixed WL Chromatogram, $254 \mathrm{~nm}$

Peak Quantitation: AREA

Calculation Method: EXT-STD

Scale Factor 1: 1.000

\begin{tabular}{|c|c|c|c|c|}
\hline No. & $\mathrm{RT}$ & Area & Height & Area 응 \\
\hline 1 & 67.03 & 1951283 & 13819 & 34.588 \\
\hline 2 & 74.26 & 3690285 & 23583 & 65.412 \\
\hline & & 5641568 & 37402 & 100.000 \\
\hline
\end{tabular}

Peak rejection level: 1000 